\title{
$\alpha$-Glucosidase Inhibitory Activities and the Interaction Mechanism of Novel Spiro-Flavoalkaloids from YingDe Green Tea
}

Zhi-Wei Hou, Chen-Hui Chen, Jia-Ping Ke, Yuan-Yuan Zhang, Yan Qi, Shi-Yu Liu,

Zi Yang, Jing-Ming Ning, Guan-Hu Bao*

State Key Laboratory of Tea Plant Biology and Utilization, Anhui Agricultural

University, Hefei, Anhui Province 230036, China

Figure S1. The manufacturing processes of Yingde green tea.

Figure S2. Extraction and isolation of YDGT and purification flow chart of compounds

\section{1-4.}

Figure S3. UV spectra of compounds 1, 2, 3 and 4.

Figure S4. IR spectra of compound 1.

Figure S5. ${ }^{1} \mathrm{H}$ NMR spectra of compound 1 in DMSO- $d_{6}$.

Figure S6. ${ }^{13} \mathrm{C}$ NMR spectra of compound 1 in DMSO- $d_{6}$.

Figure S7. HSQC spectra of compound 1 in DMSO- $d_{6}$.

Figure S8. HMBC spectra of compound 1 in DMSO- $d_{6}$.

Figure S9. COSY spectra of compound 1 in DMSO- $d_{6}$.

Figure S10. NOESY spectra of compound 1 in DMSO- $d_{6}$.

Figure S11. DEPT-135 spectra of compound 1 in DMSO- $d_{6}$.

Figure S12. Local DEPT-135 and ${ }^{13} \mathrm{C}$ spectrum of compound 1 in DMSO- $d_{6}$.

Figure S13. Local NOESY spectra of compound 1 in DMSO- $d_{6}$.

Figure S14. CD spectra of compound 1, 2, 3 and 4 in $\mathrm{MeOH}$.

Figure S15. IR spectra of compound 2.

Figure S16. ${ }^{1} \mathrm{H}$ NMR spectra of compound 2 in DMSO- $d_{6}$.

Figure S17. ${ }^{13} \mathrm{C}$ NMR spectra of compound 2 in DMSO- $d_{6}$. 
Figure S18. HSQC spectra of compound 2 in DMSO- $d_{6}$.

Figure S19. HMBC spectra of compound 2 in DMSO- $d_{6}$.

Figure S20. COSY spectra of compound 2 in DMSO- $d_{6}$.

Figure S21. ROESY spectra of compound 2 in DMSO- $d_{6}$.

Figure S22. DEPT-135 spectra of compound 2 in DMSO- $d_{6}$.

Figure S23. IR spectra of compound 3.

Figure S24. ${ }^{1} \mathrm{H}$ NMR spectra of compound 3 in DMSO- $d_{6}$.

Figure S25. ${ }^{13} \mathrm{C}$ NMR spectra of compound 3 in DMSO- $d_{6}$.

Figure S26. HSQC spectra of compound 3 in DMSO- $d_{6}$.

Figure S27. HMBC spectra of compound 3 in DMSO- $d_{6}$.

Figure S28. COSY spectra of compound 3 in DMSO- $d_{6}$.

Figure S29. NOESY spectra of compound 3 in DMSO- $d_{6}$.

Figure S30. DEPT-135 spectra of compound 3 in DMSO- $d_{6}$.

Figure S31. Local NOESY spectra of compound 3 in DMSO- $d_{6}$.

Figure S32. IR spectra of compound 4.

Figure S33. ${ }^{1} \mathrm{H}$ NMR spectra of compound 4 in DMSO- $d_{6}$.

Figure S34. ${ }^{13} \mathrm{C}$ NMR spectra of compound 4 in DMSO- $d_{6}$.

Figure S35. HSQC spectra of compound 4 in DMSO- $d_{6}$.

Figure S36. HMBC spectra of compound 4 in DMSO- $d_{6}$.

Figure S37. COSY spectra of compound 4 in DMSO- $d_{6}$.

Figure S38. NOESY spectra of compound 4 in DMSO- $d_{6}$.

Figure S39. DEPT-135 spectra of compound 4 in DMSO- $d_{6}$.

Figure S40. MS/MS spectrum of compound 1, 2, 3 and 4.

Figure S41. 3D molecular model of compounds 1,2, 3, and 4 showing key NOESY (dashed double arrowhead line) correlations.

Figure S42. Key NOESY correlations between $\mathrm{H}-2$ and $\mathrm{H}_{2}-4$ for compounds $\mathbf{1 - 4}$.

Figure S43. Local ${ }^{1} \mathrm{H}$ NMR spectra of compound 3 before (brown line) and after (green line) the storage in DMSO- $d_{6}$.

Figure S44. (A) TIC of the $70 \%$ aqueous $\mathrm{MeOH}$ extract of YDGT fresh leaves compared with the TIC of compounds $1,2,3$ and 4. (B) HPLC chromatogram of the 
$70 \%$ aqueous $\mathrm{MeOH}$ extract of YDGT fresh leaves compared with the HPLC chromatogram of compounds $1,2,3$ and 4 .

Figure S45. TIC of flavoalkaloids ([M-H] $]^{-}$568.14, [M-H $\left.]^{-} 552.15\right)$ and compounds $\mathbf{1}$, 2,3 and 4 .

Figure S46. TIC of the $\mathrm{MeOH}$ fractions of green tea (Camellia sinensis var. assamica.) after extraction with PE and EtOAc.

Figure S47. Possible formation route of spiro-flavoalkaloids in tea processing.

Figure S48. Interactions of acarbose with $\alpha$-glucosidase and the 3D structure of $\alpha$ glucosidase-acarbose complex with the key residues.

The detailed information of fluorescence quenching experiment.

The detailed information of $\mathrm{UV}-\mathrm{Vis}$ spectroscopy measurements.

Table S1. The inhibitory effect of flavonoids on $\alpha$-glucosidase in previous reports.

Table S2. DP4+ probability of ${ }^{13} \mathrm{C}$ NMR data of the isomers (1).

Table S3. Important thermodynamic parameters (a.u.) and Boltzmann distributions of the optimized $\left(2 R^{*}, 3 R^{*}, 5^{\prime \prime \prime} S^{*}\right)-1$ at B3LYP/6-31G (d) level in methanol.

Table S4. Optimized coordinate of $\left(2 R^{*}, 3 R^{*}, 5^{\prime \prime \prime} S^{*}\right)-1$ at B3LYP/6-31G (d) level in methanol.

Table S5. Optimized coordinate of $\left(2 R^{*}, 3 R^{*}, 5^{\prime \prime \prime} R^{*}\right)-1$ at B3LYP/6-31G (d) level in methanol. 
Figure S1. The manufacturing processes of Yingde green tea.

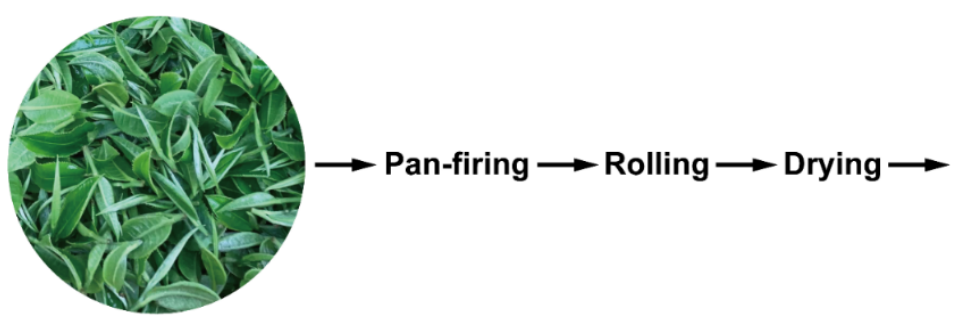

Fresh leaves (Cultivar Yinghong No.9)

(Camellia sinensis var. assamica.) 
Figure S2. Extraction and isolation of YDGT and purification flow chart of compounds 1-4.

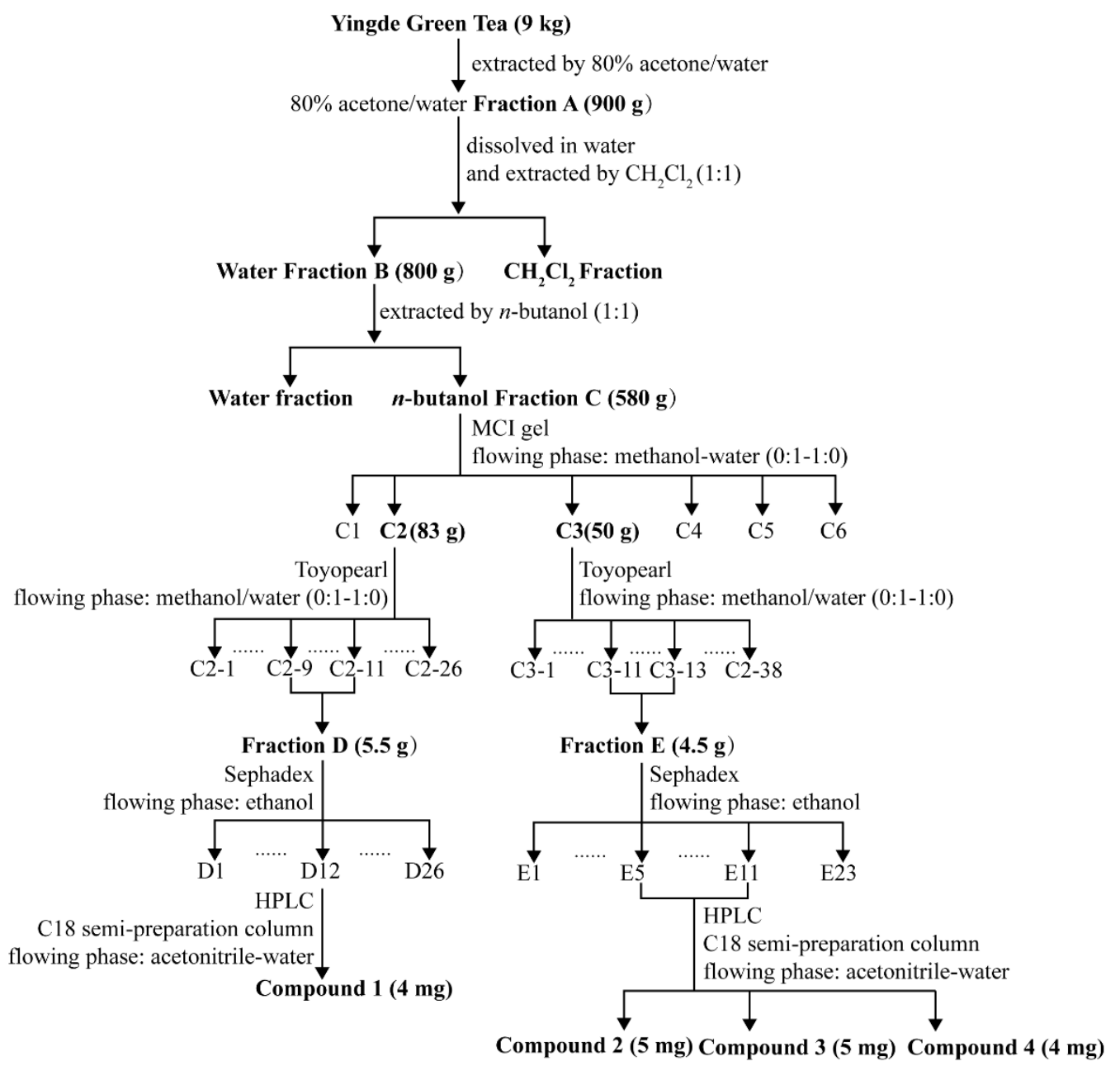


Figure S3. UV spectrum of compound 1, 2, 3 and 4.
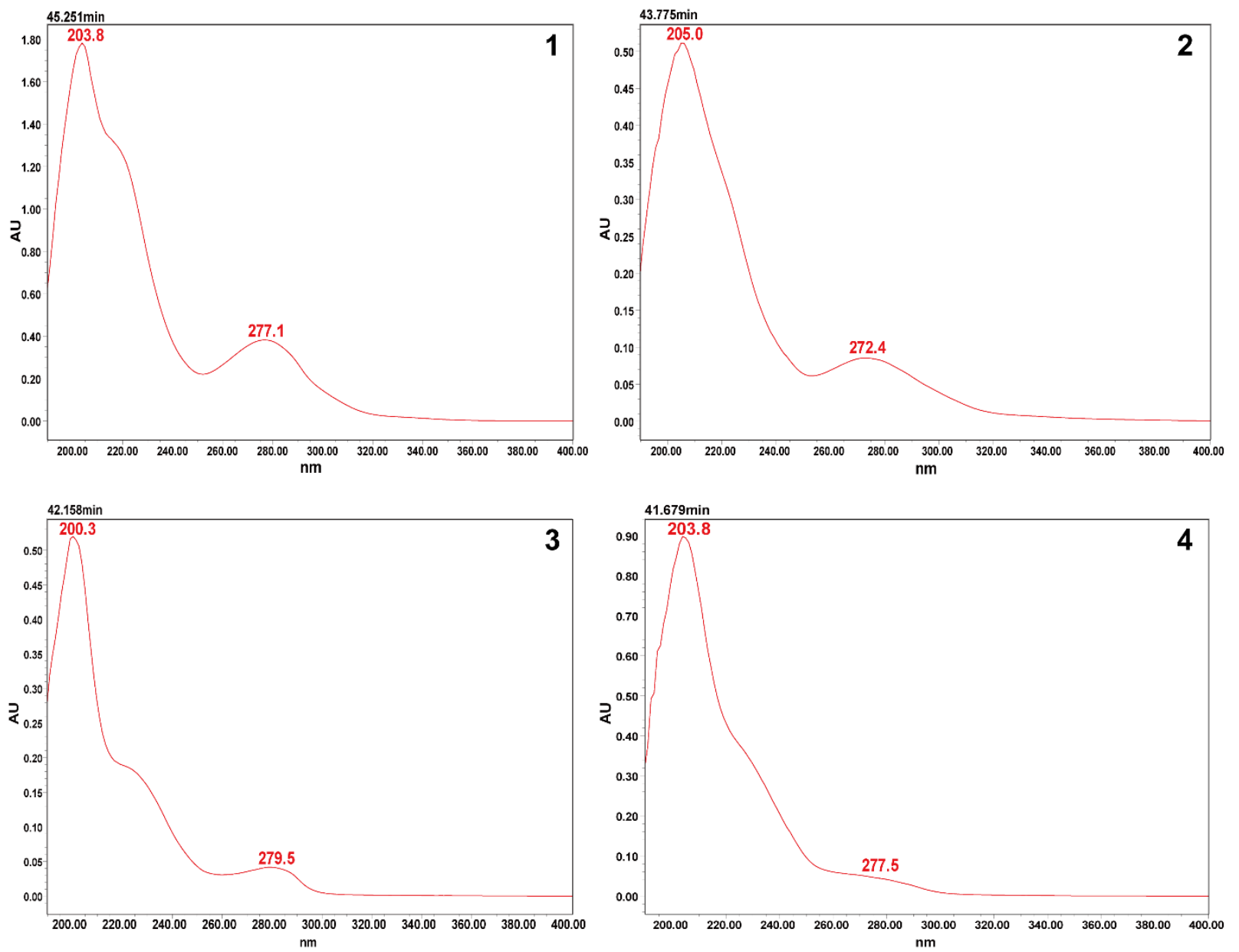
Figure S4. IR spectrum of compound 1.

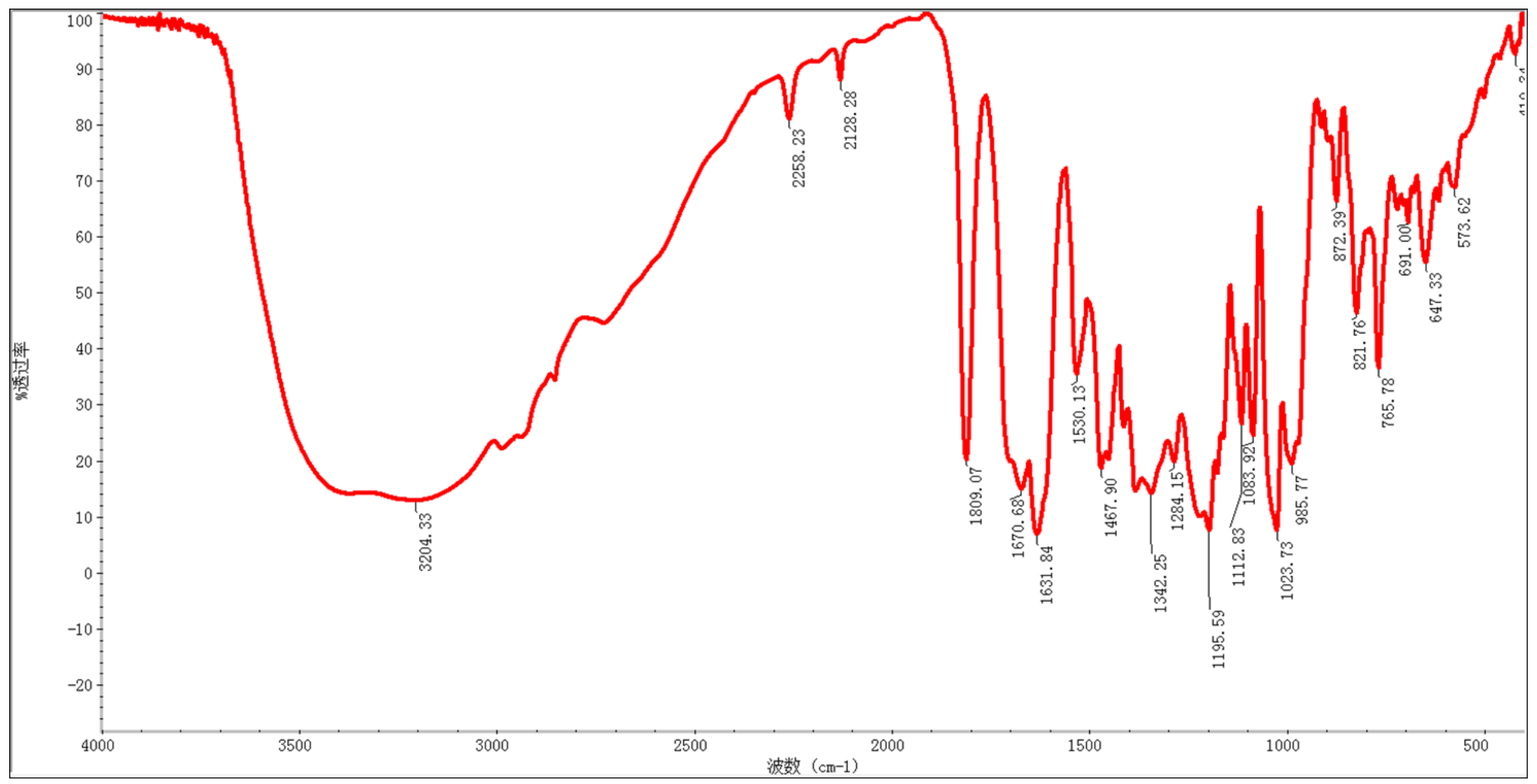


Figure S5. ${ }^{1} \mathrm{H}$ NMR spectra of compound 1 in DMSO- $d_{6}$.

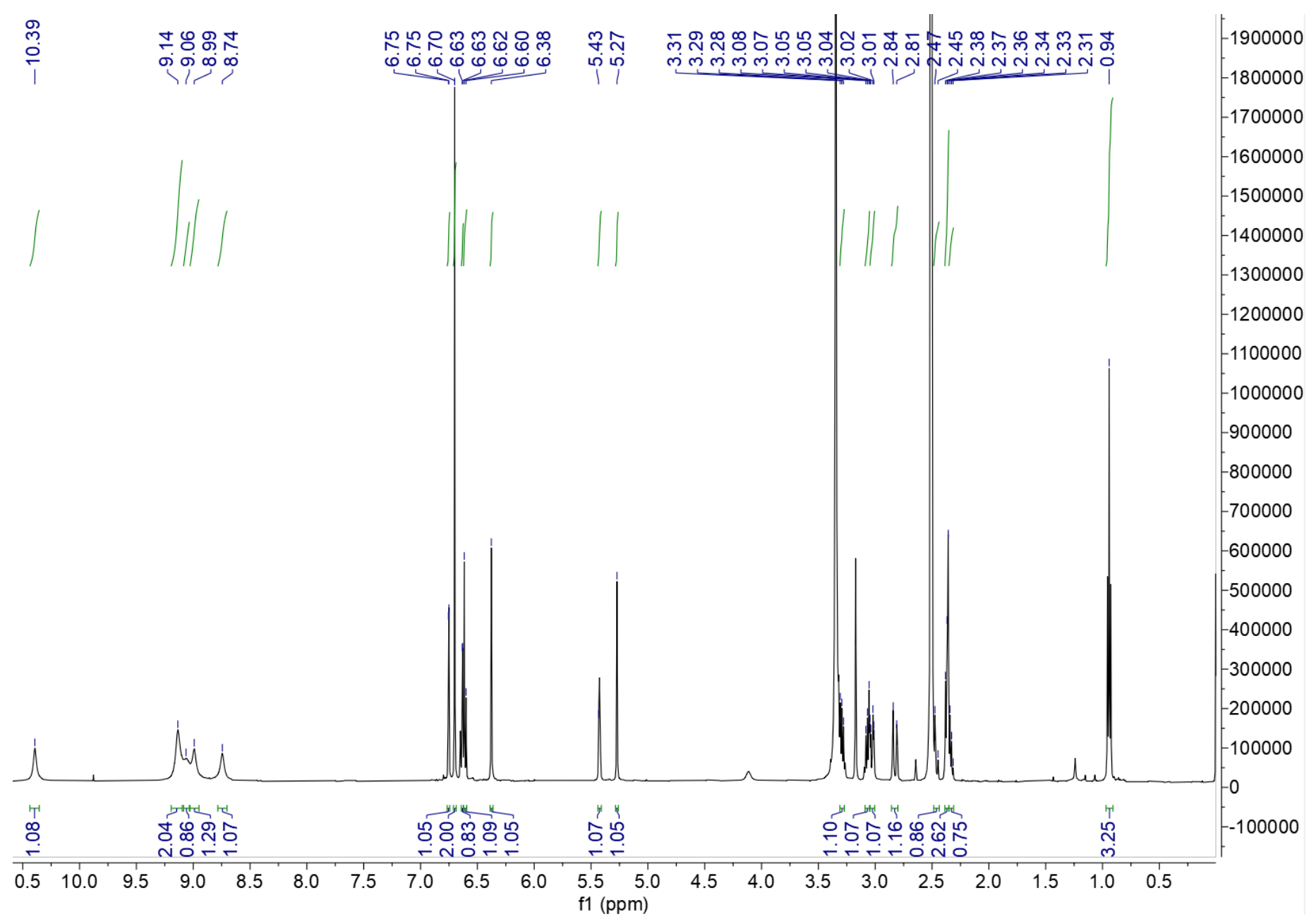


Figure S6. ${ }^{13} \mathrm{C}$ NMR spectra of compound 1 in DMSO- $d_{6}$.

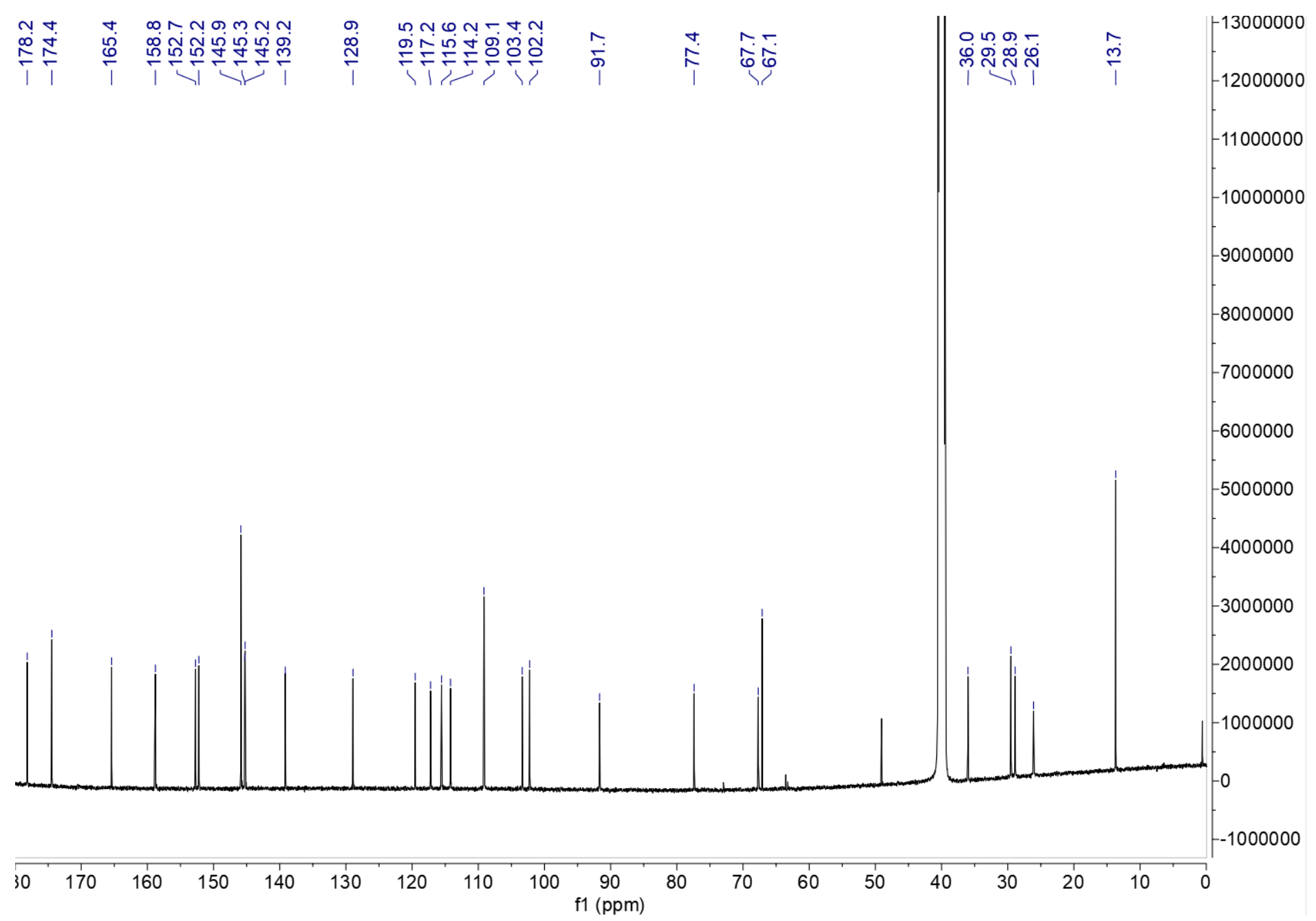


Figure S7. HSQC spectra of compound 1 in DMSO- $d_{6}$.

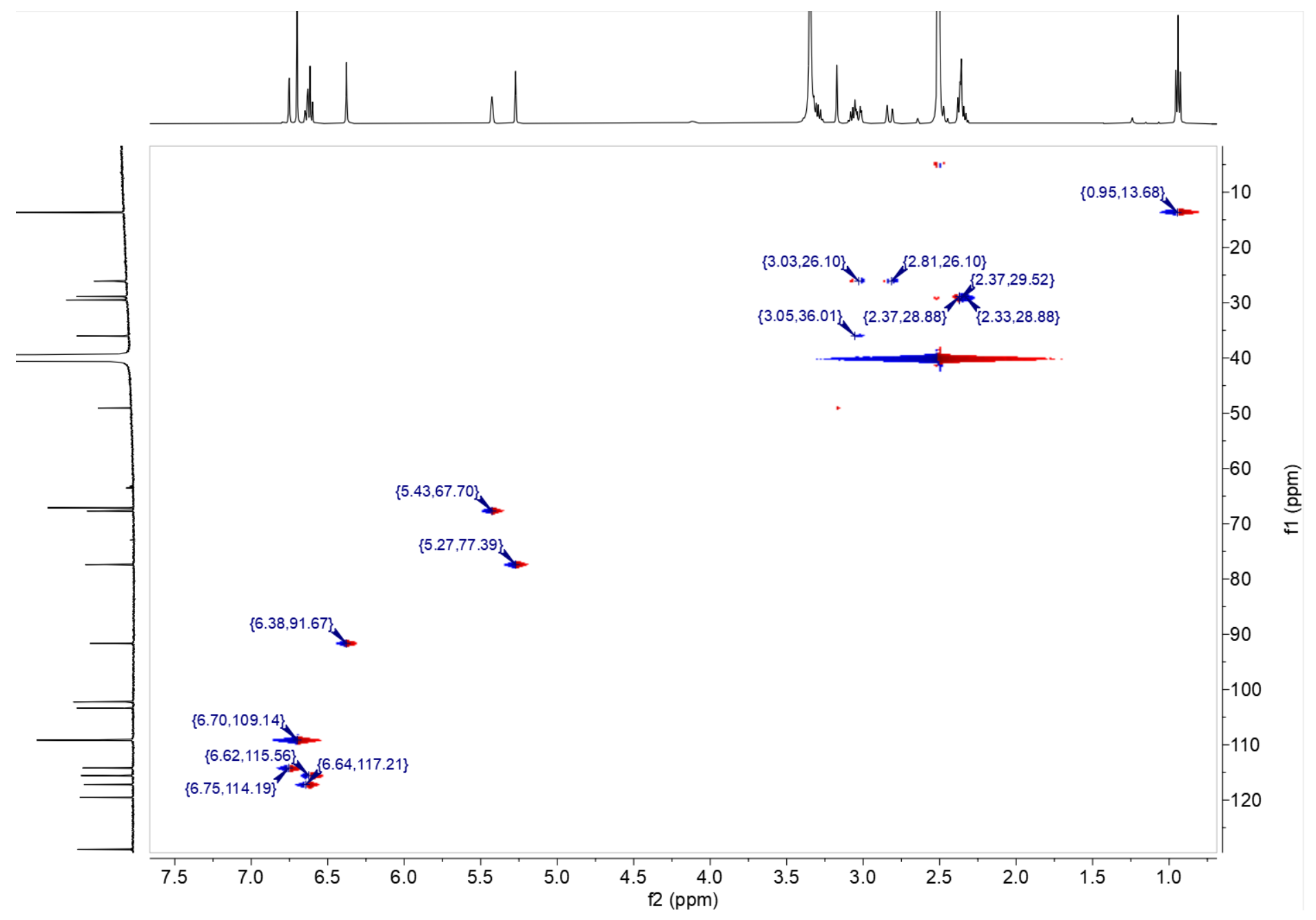


Figure S8. HMBC spectra of compound 1 in DMSO- $d_{6}$.

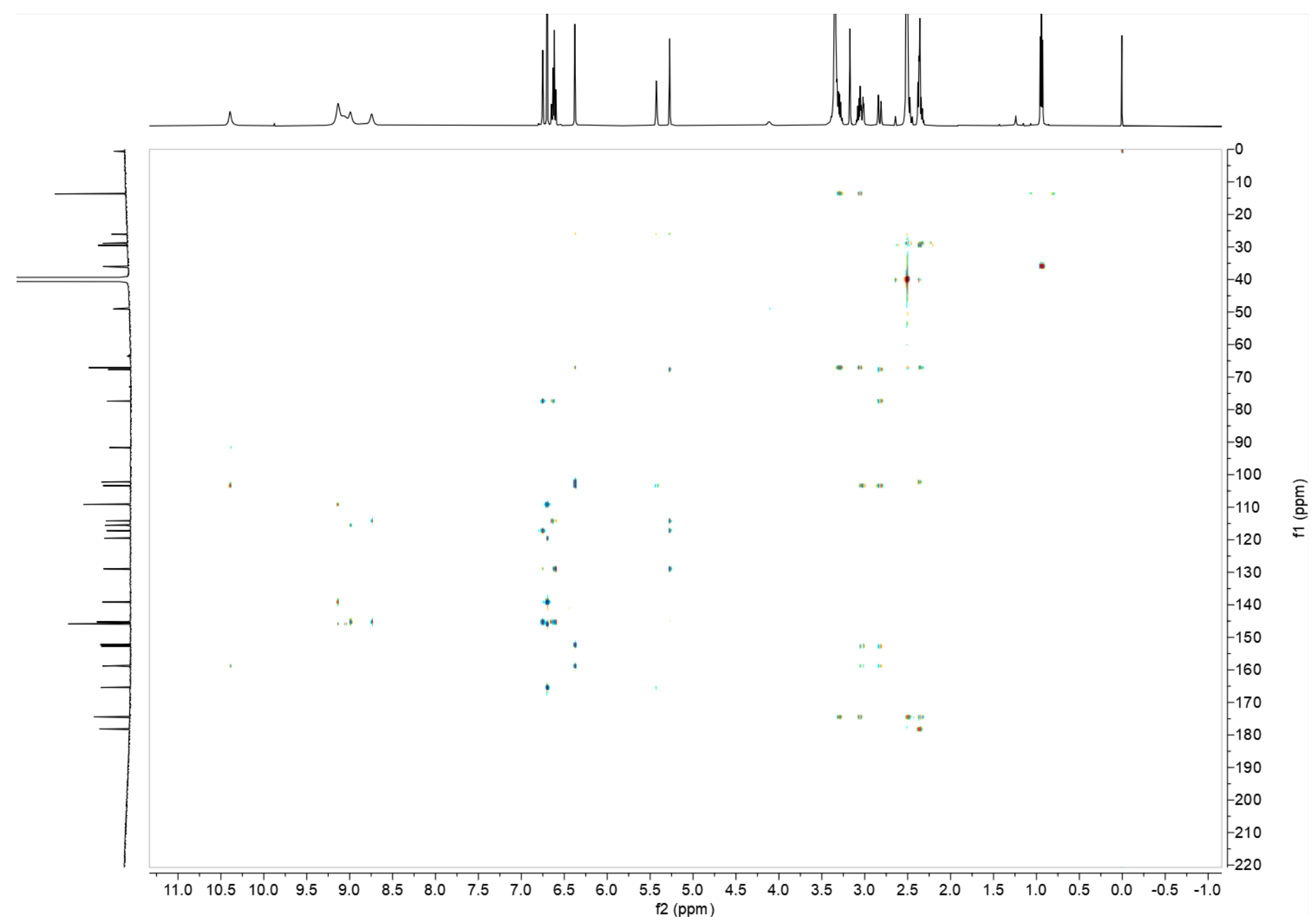


Figure S9. COSY spectra of compound 1 in DMSO- $d_{6}$.

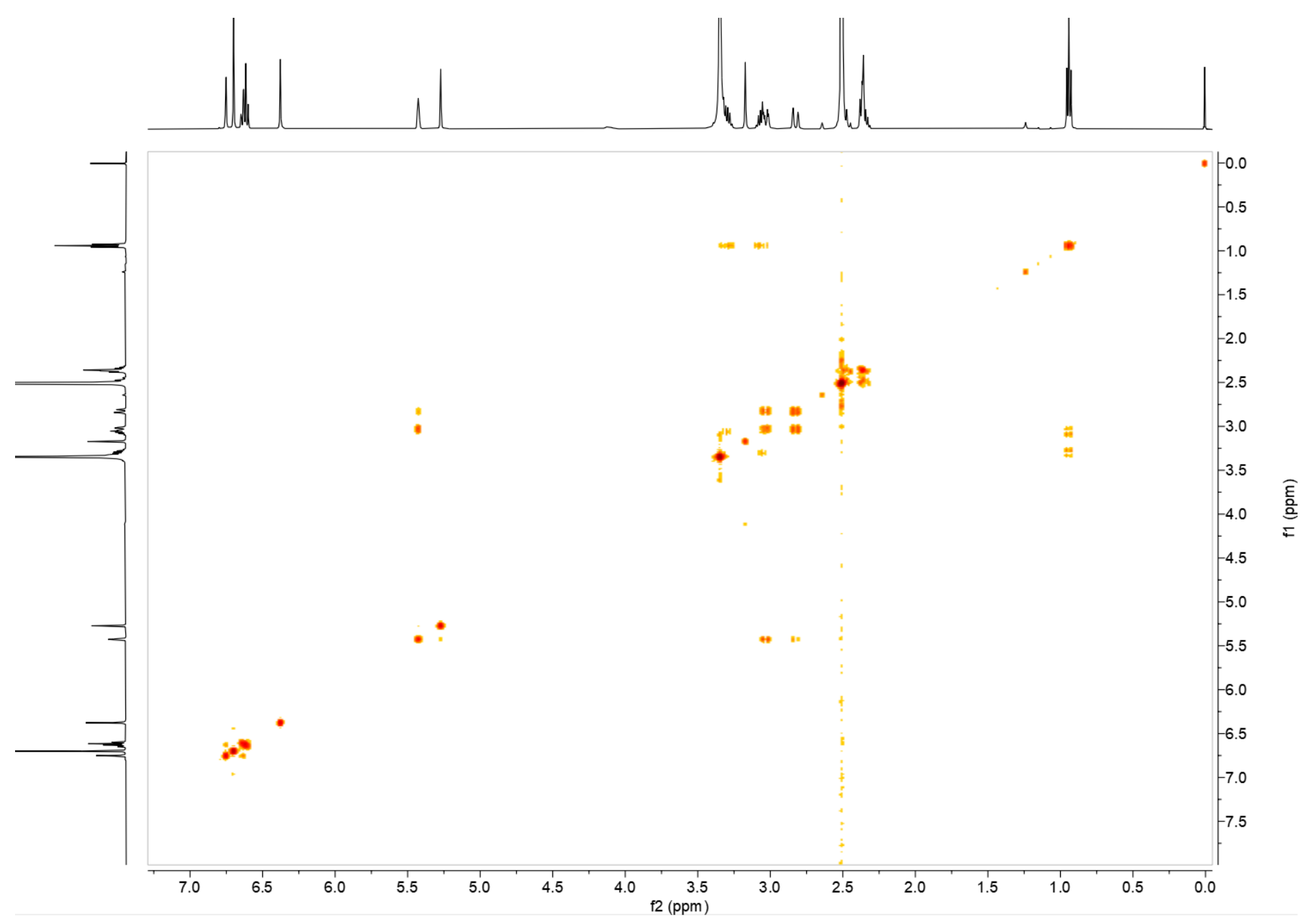


Figure S10. NOESY spectra of compound 1 in DMSO- $d_{6}$.

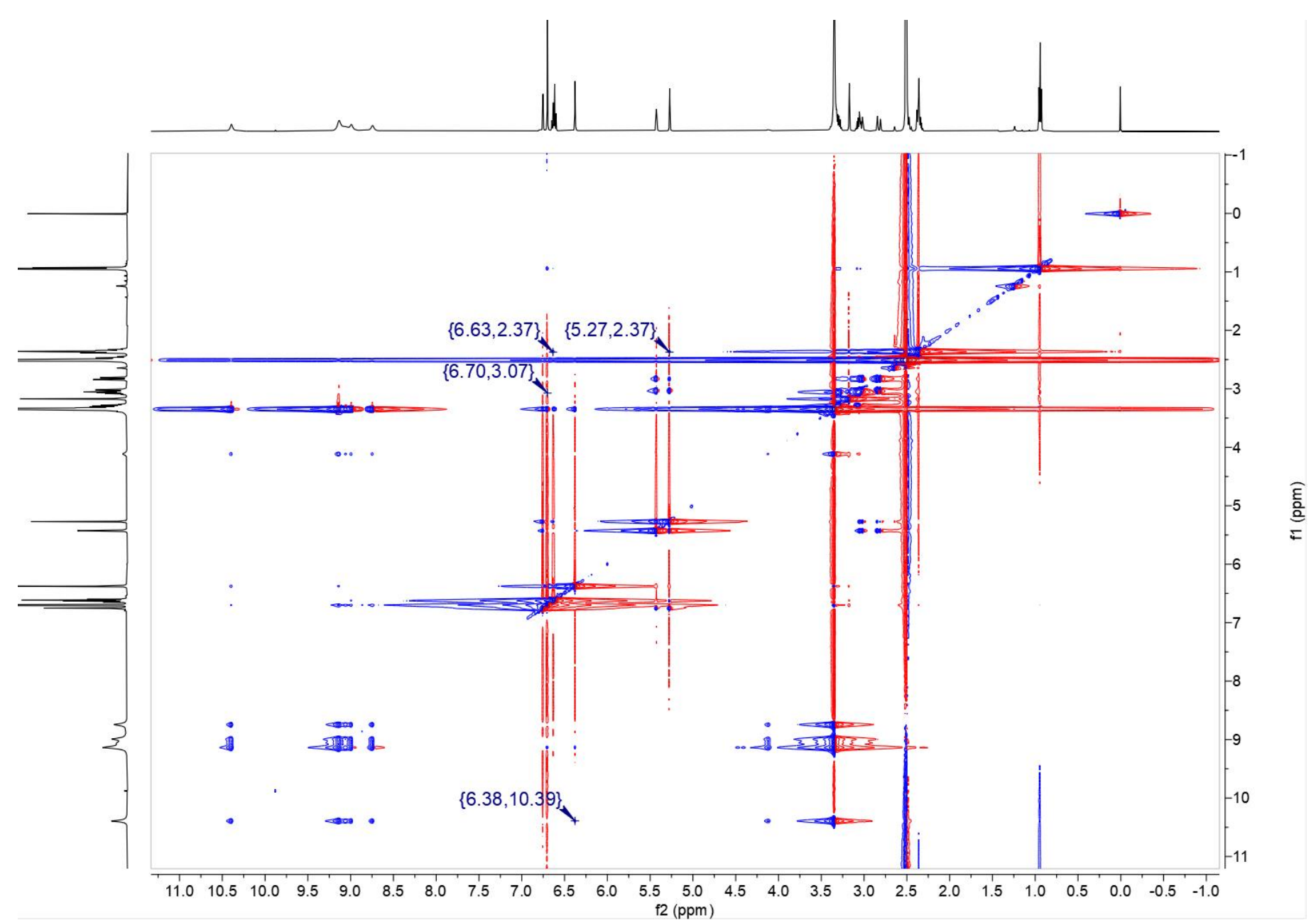


Figure S11. DEPT-135 spectra of compound 1 in DMSO- $d_{6}$.

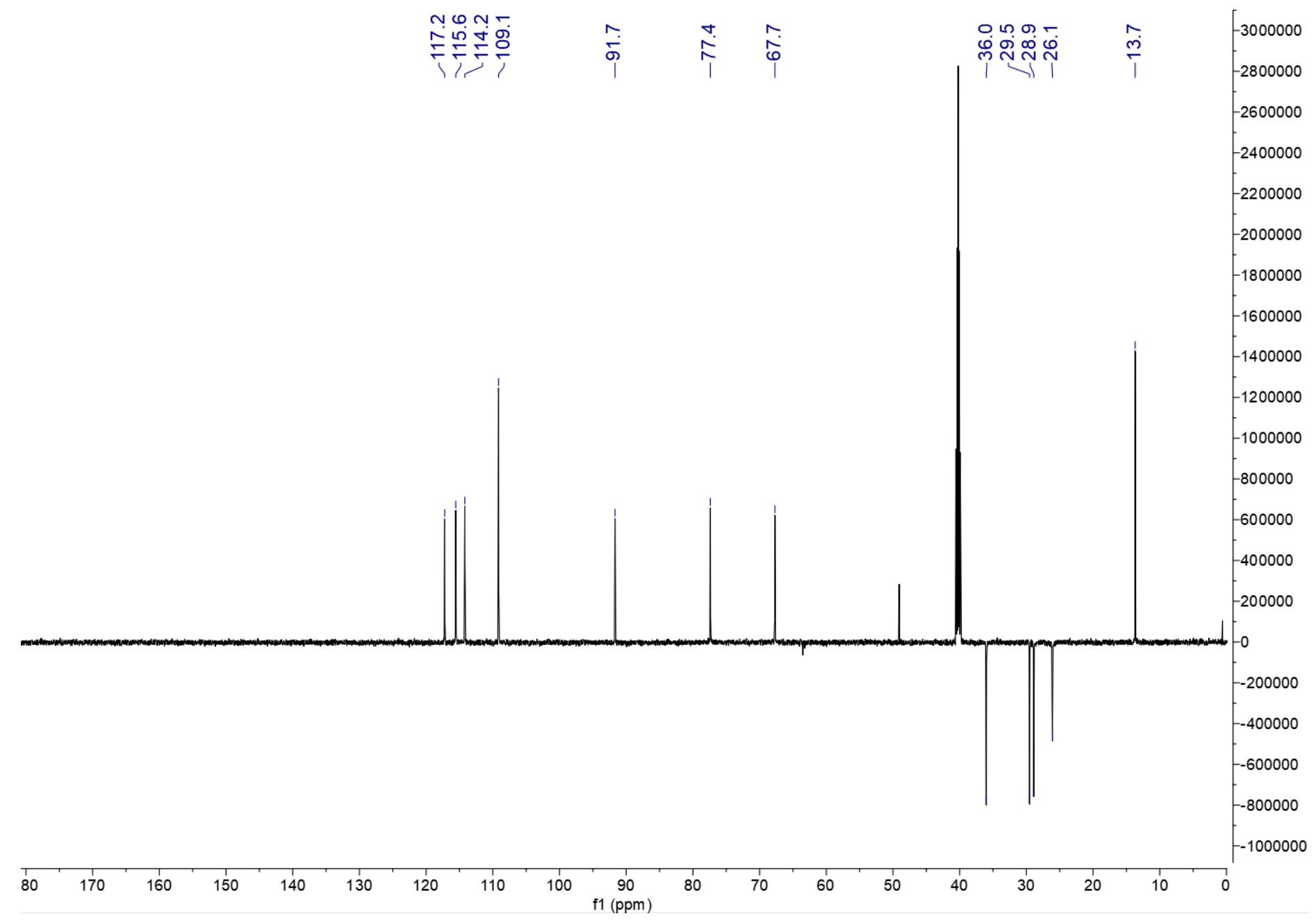


Figure S12. Local DEPT-135 and ${ }^{13} \mathrm{C}$ spectrum of compound 1 in DMSO- $d_{6}$.

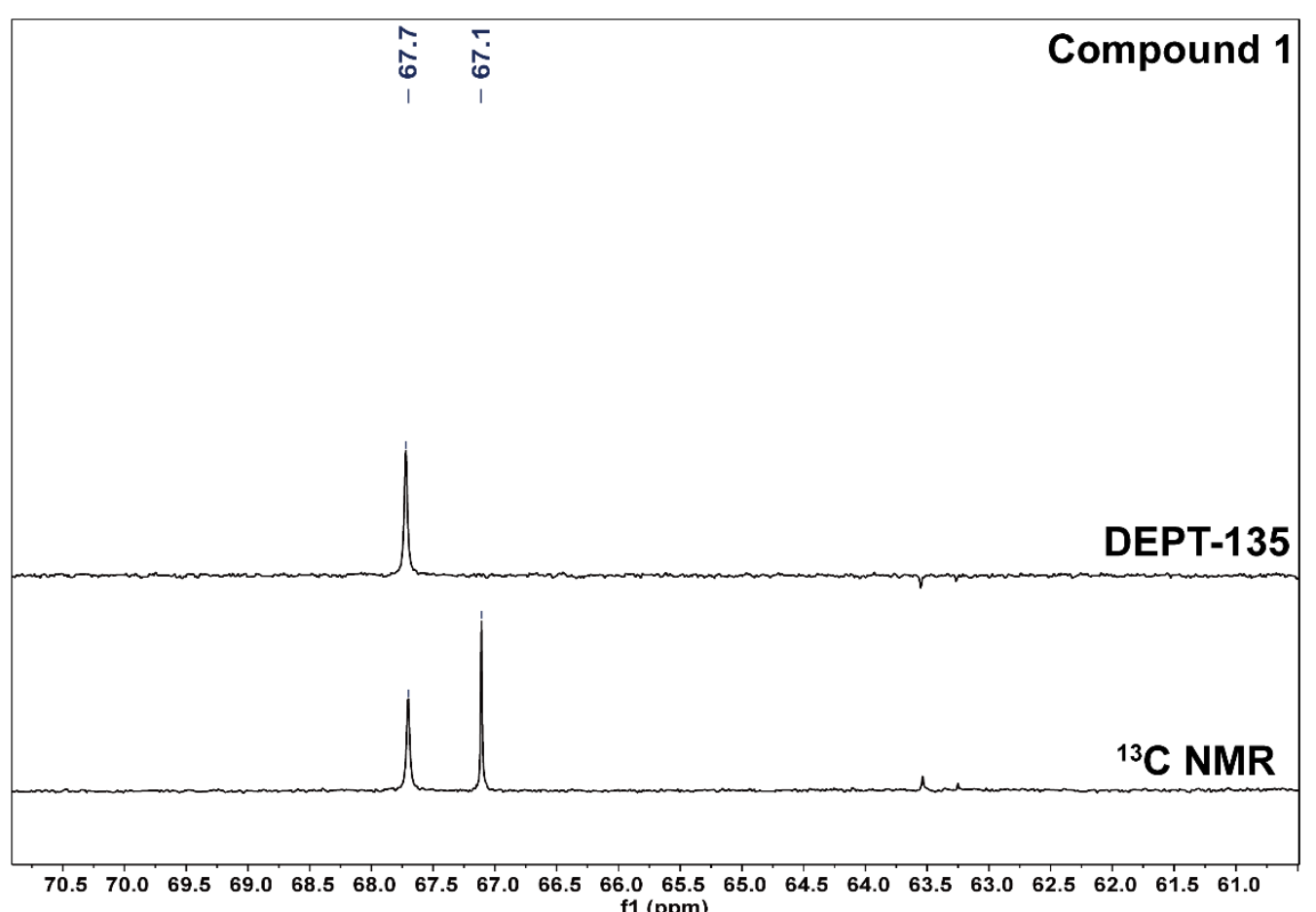
$\mathrm{f} 1(\mathrm{ppm})$ 
Figure S13. Local NOESY spectra of compound 1 in DMSO- $d_{6}$.

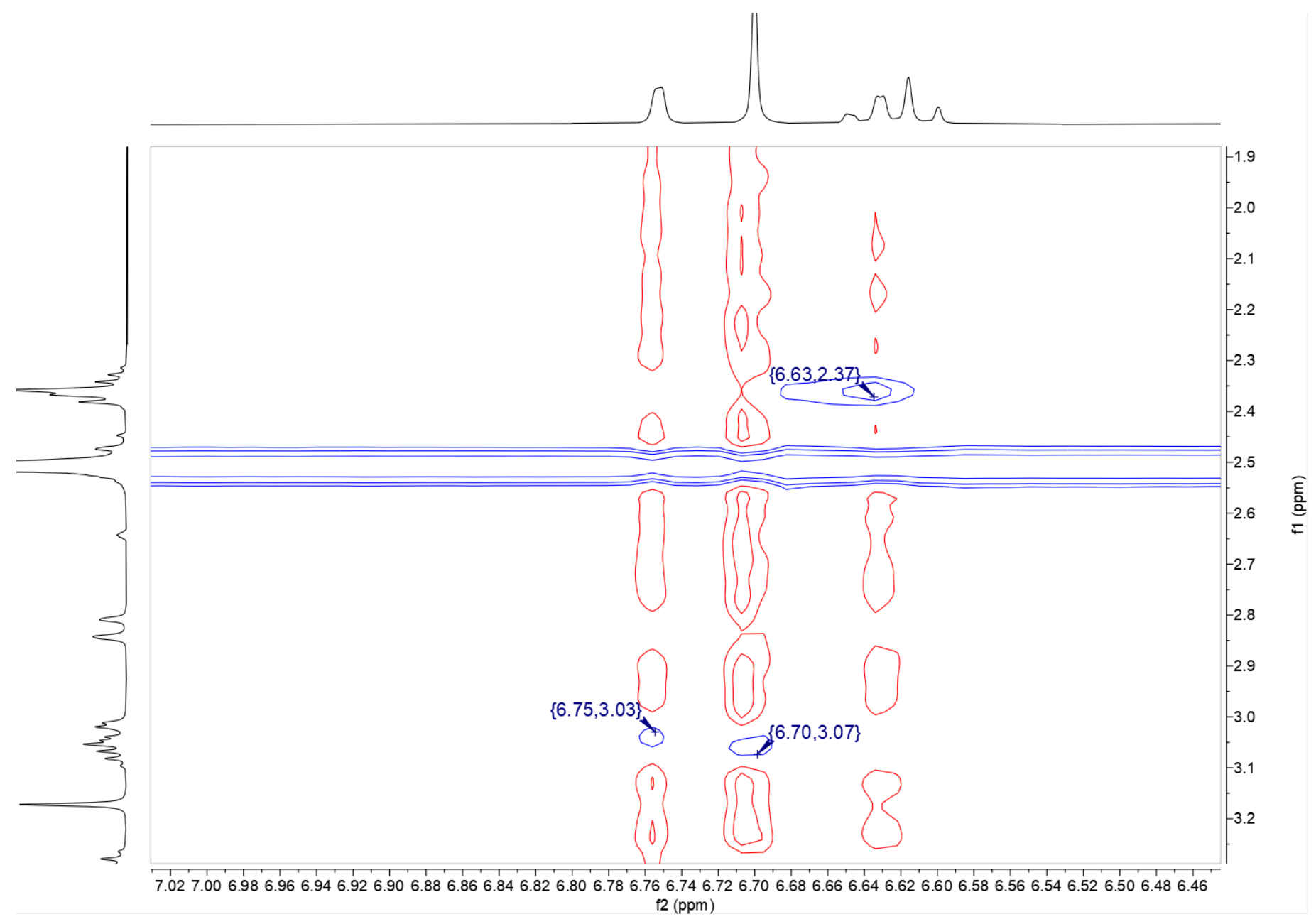


Figure S14. CD spectra of compound 1, 2, 3 and 4 in $\mathrm{MeOH}$.
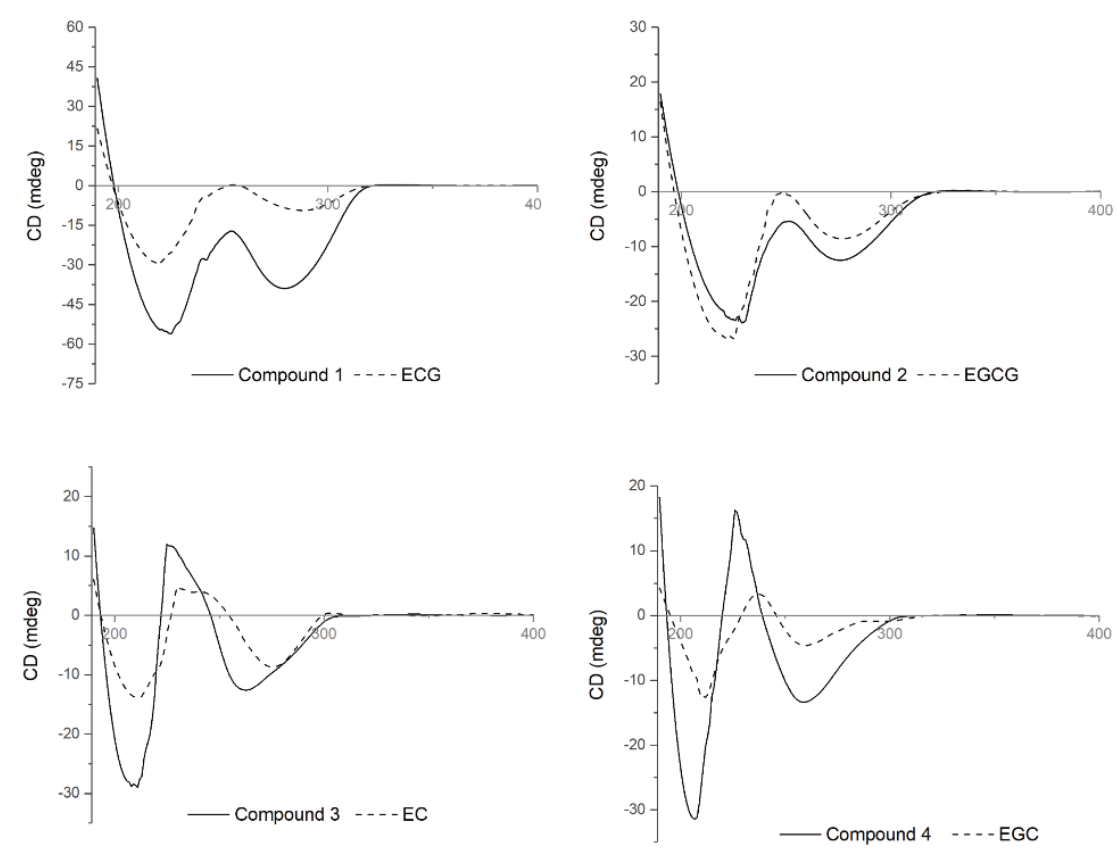
Figure S15. IR spectrum of compound 2.

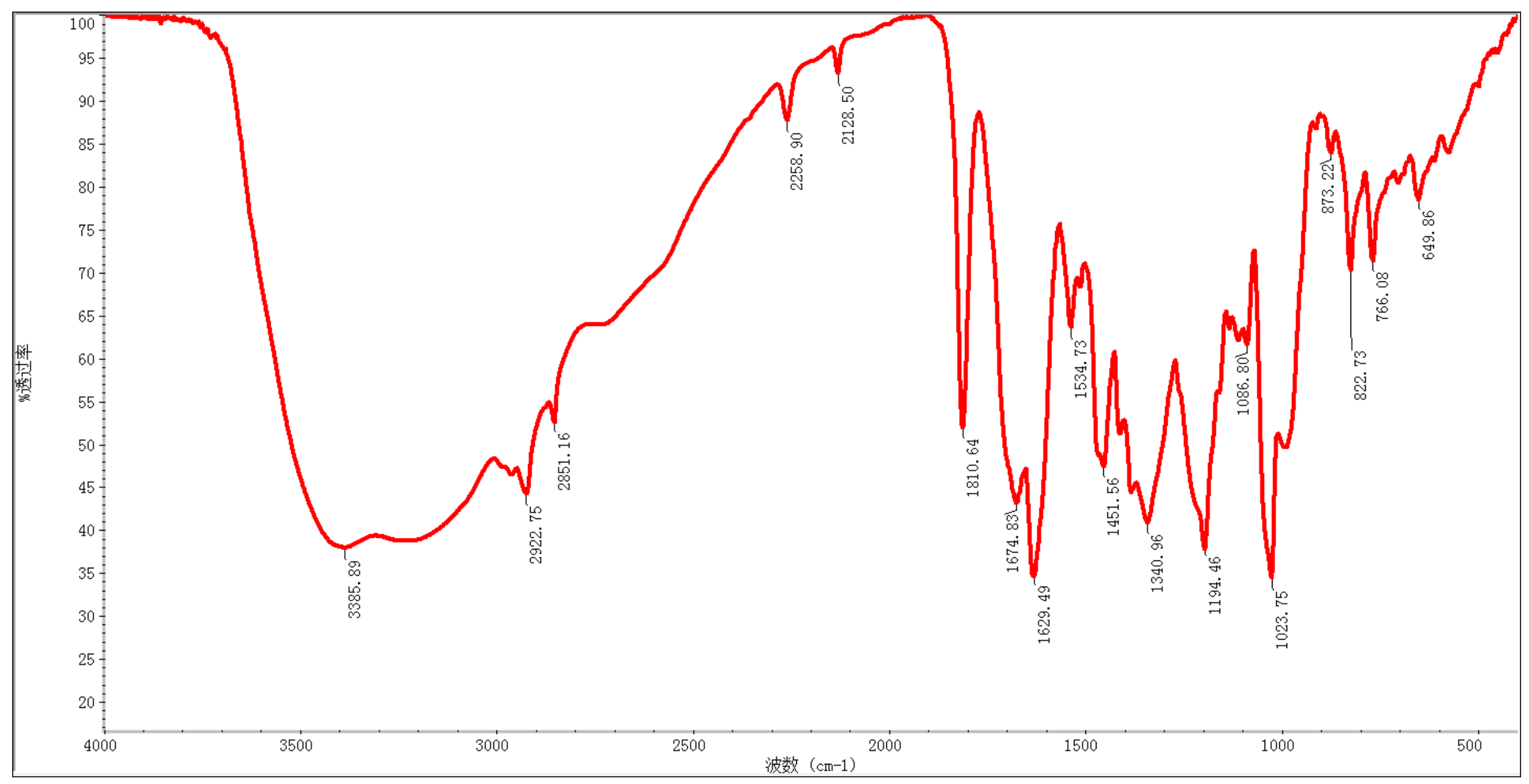


Figure S16. ${ }^{1} \mathrm{H}$ NMR spectra of compound 2 in DMSO- $d_{6}$.

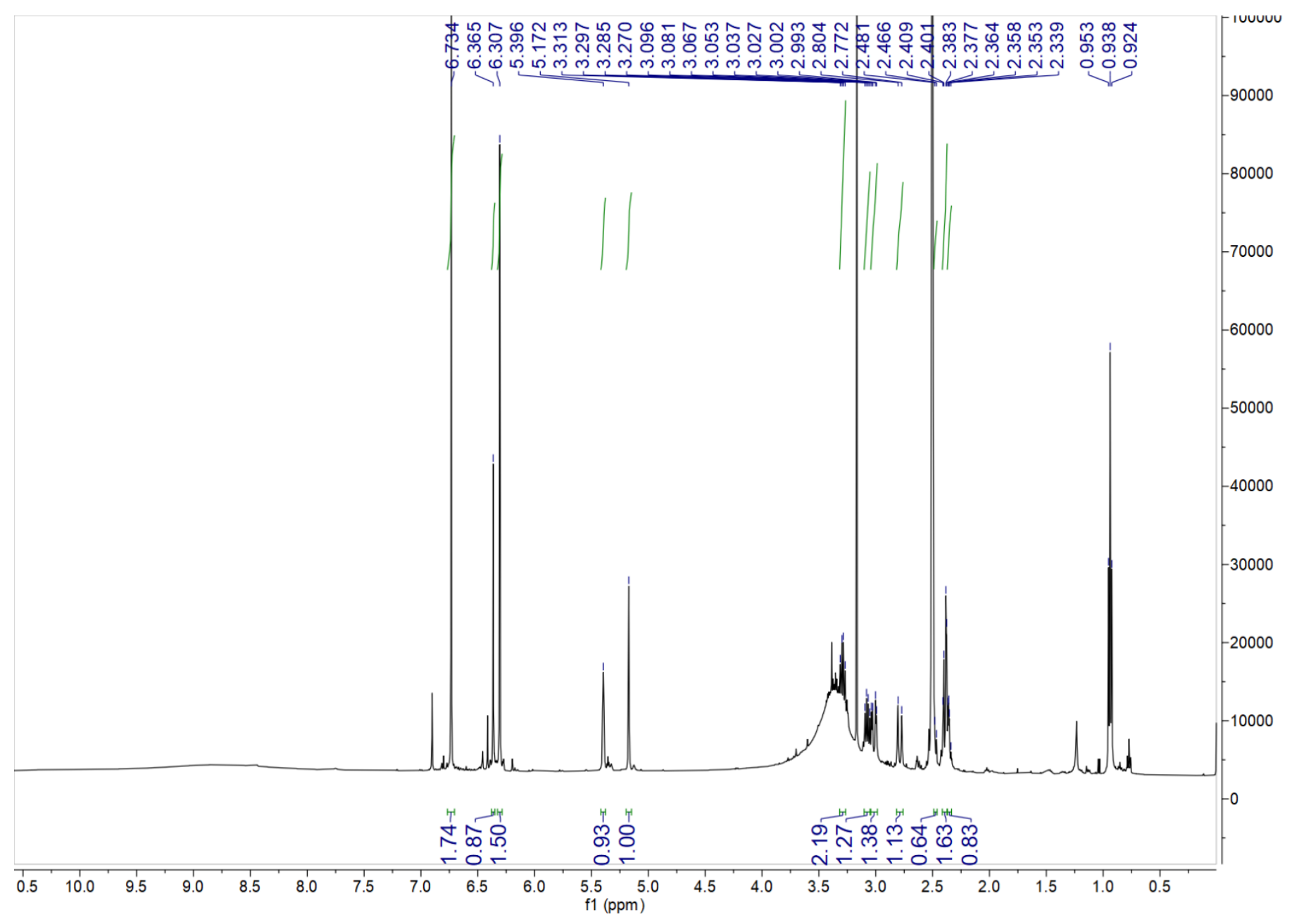


Figure S17. ${ }^{13} \mathrm{C}$ NMR spectra of compound 2 in DMSO- $d_{6}$.

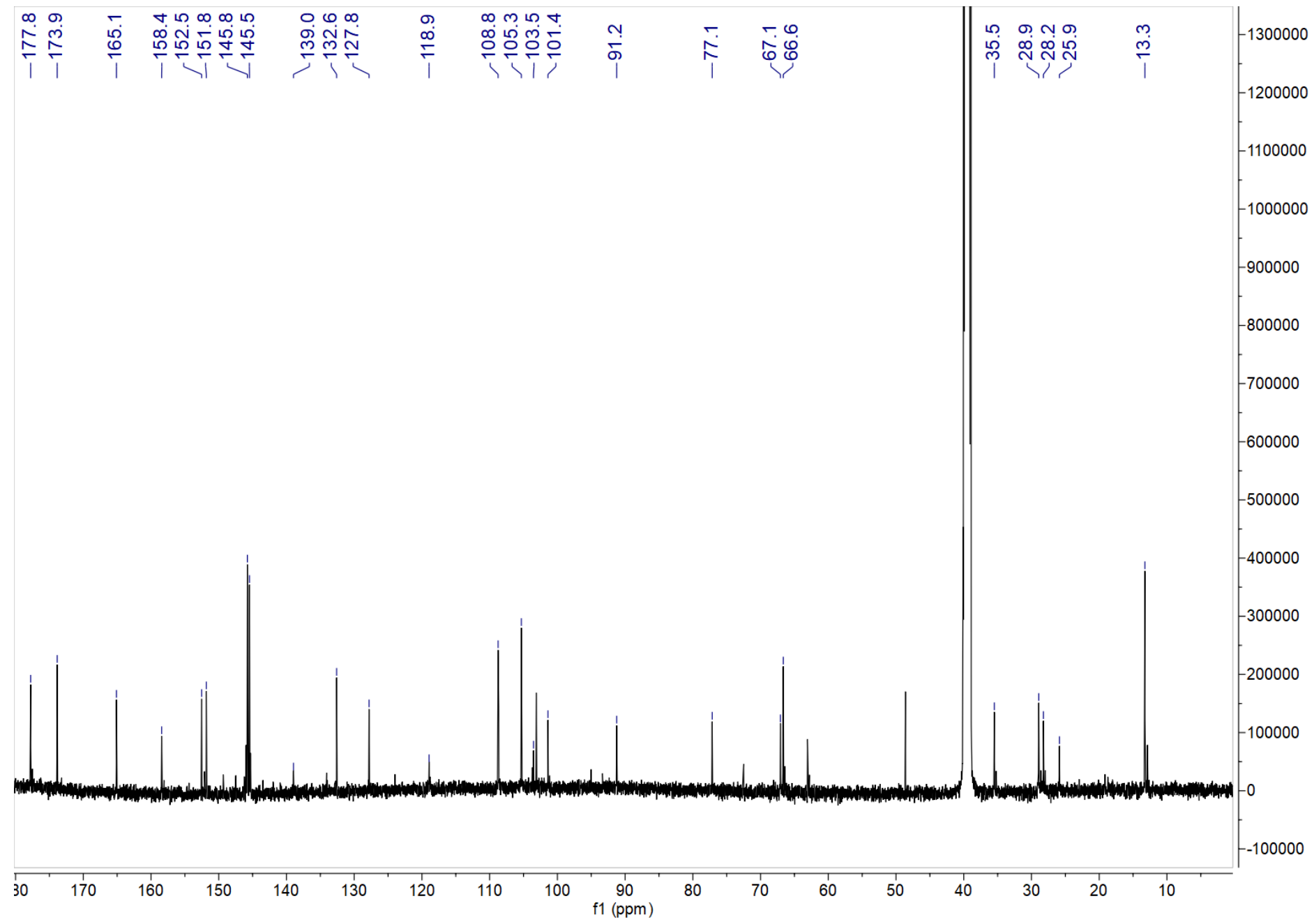


Figure S18. HSQC spectra of compound 2 in DMSO- $d_{6}$.

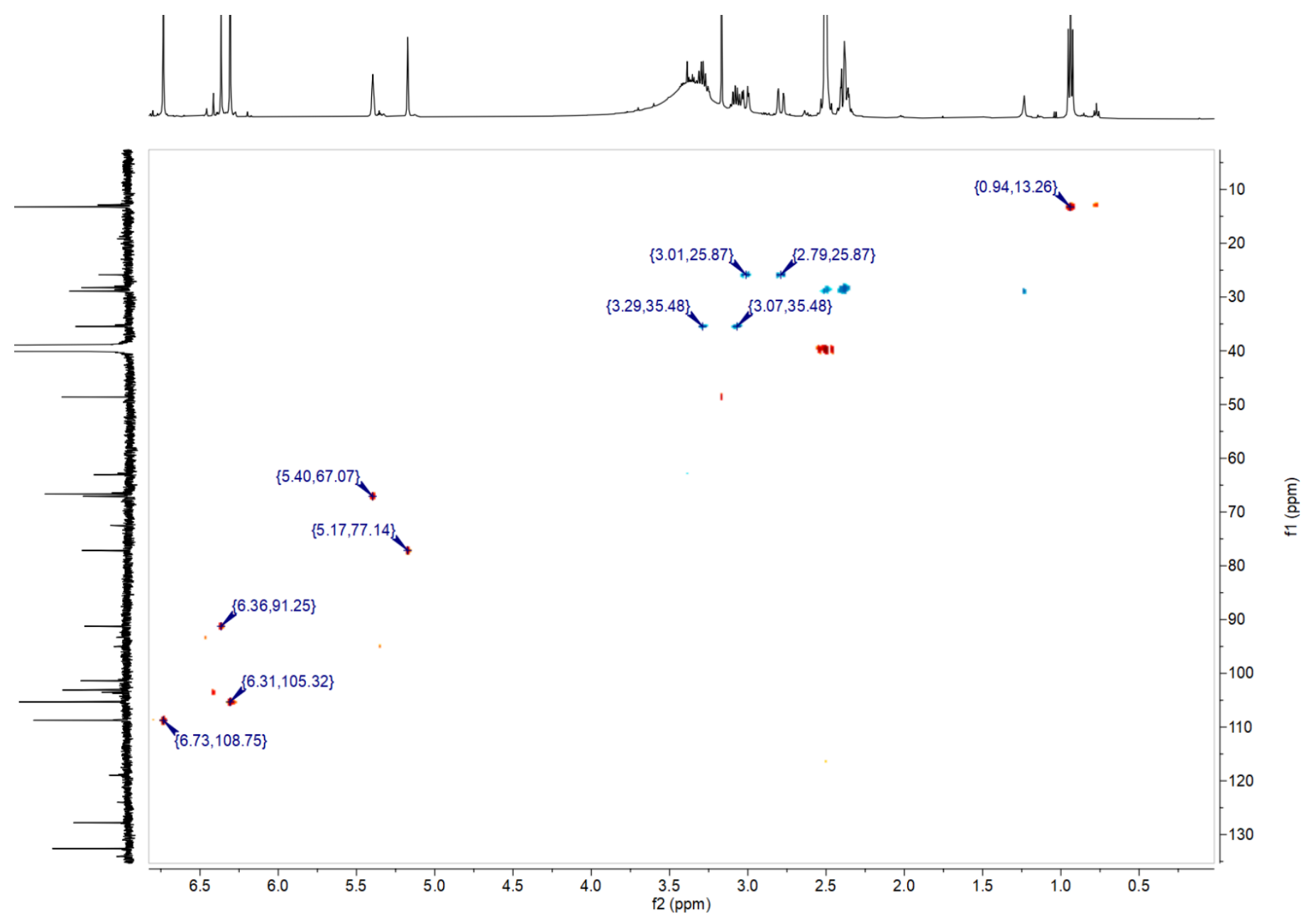


Figure S19. HMBC spectra of compound 2 in DMSO- $d_{6}$.

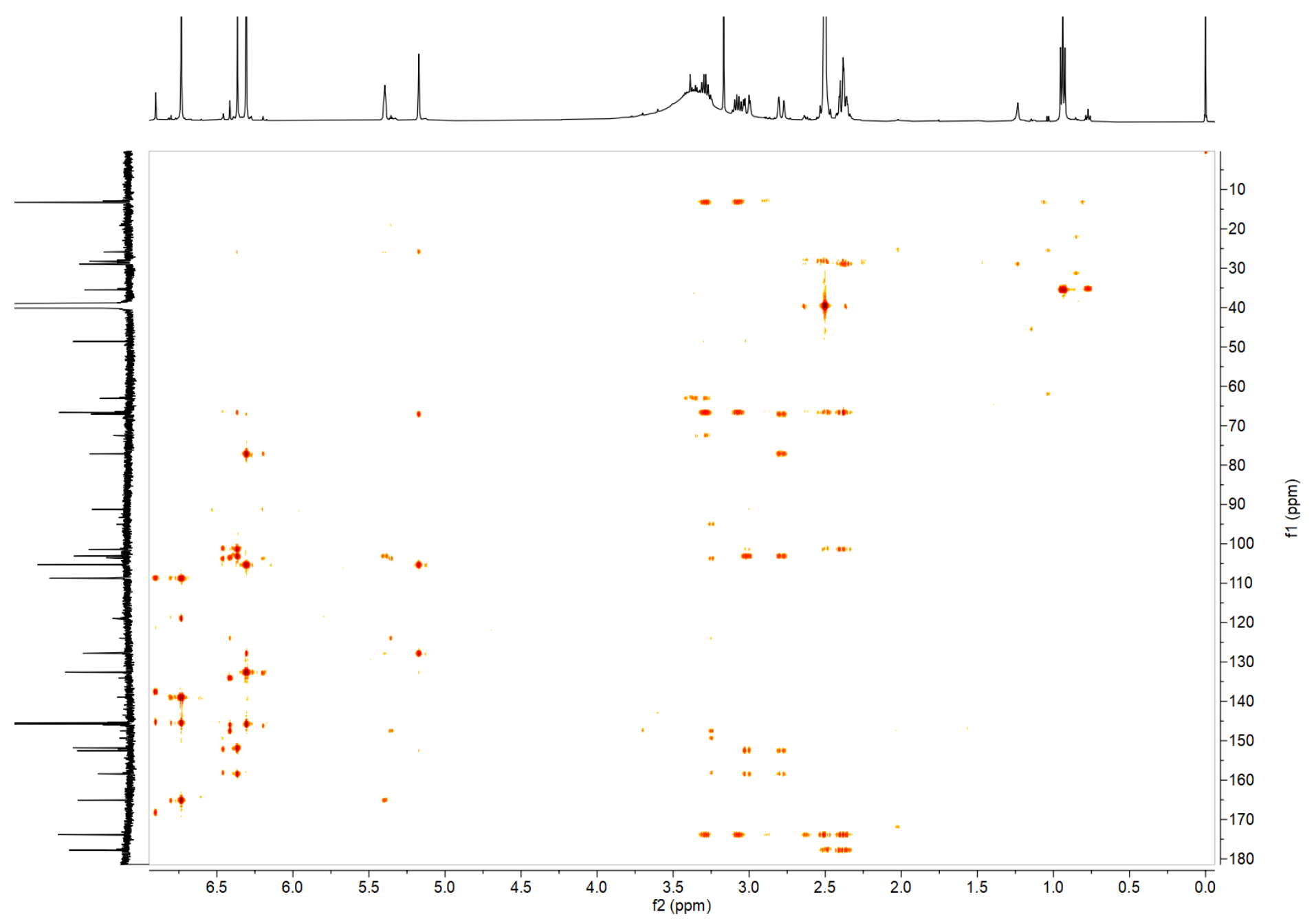


Figure S20. COSY spectra of compound 2 in DMSO- $d_{6}$.

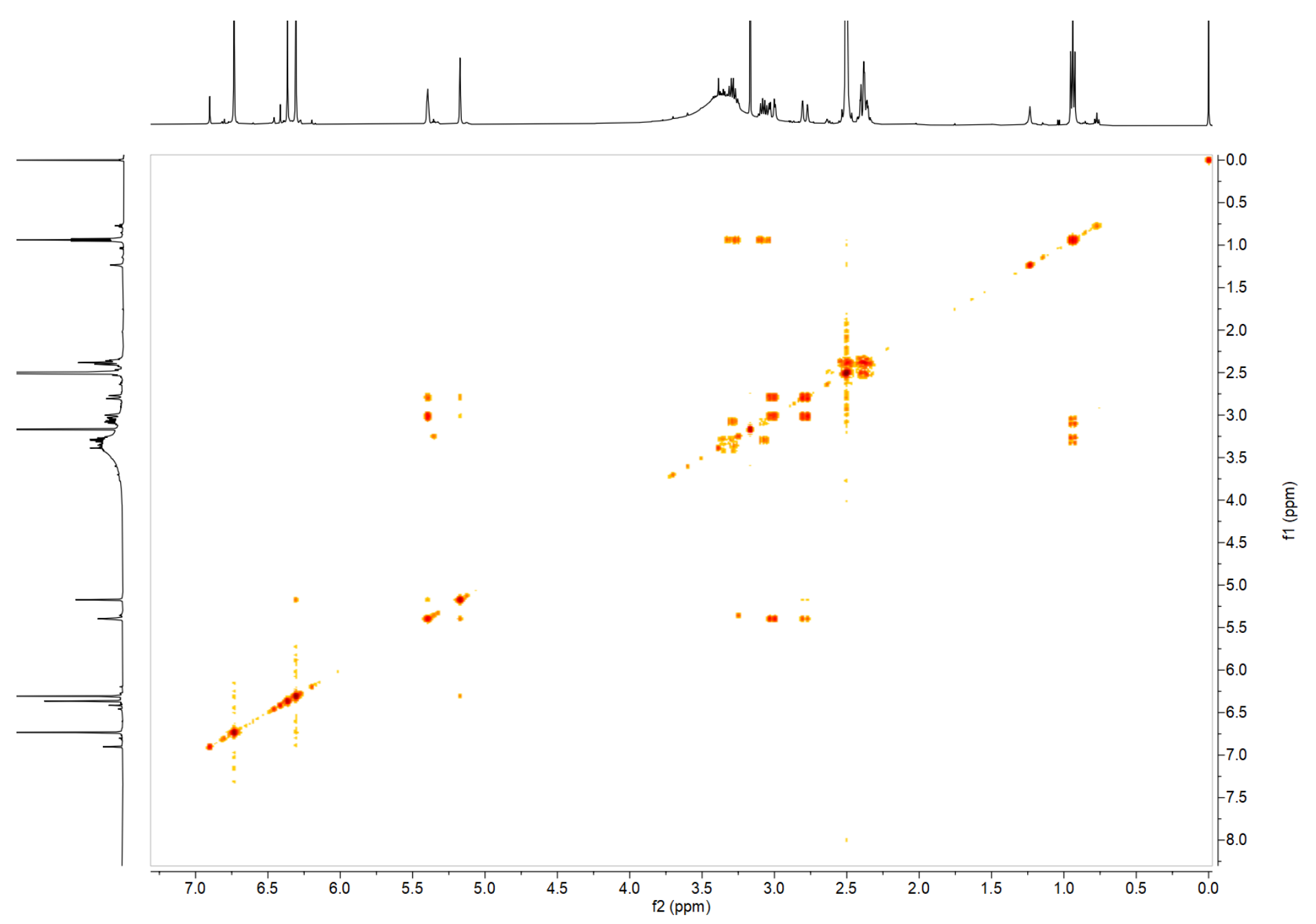


Figure S21. ROESY spectra of compound 2 in DMSO- $d_{6}$.

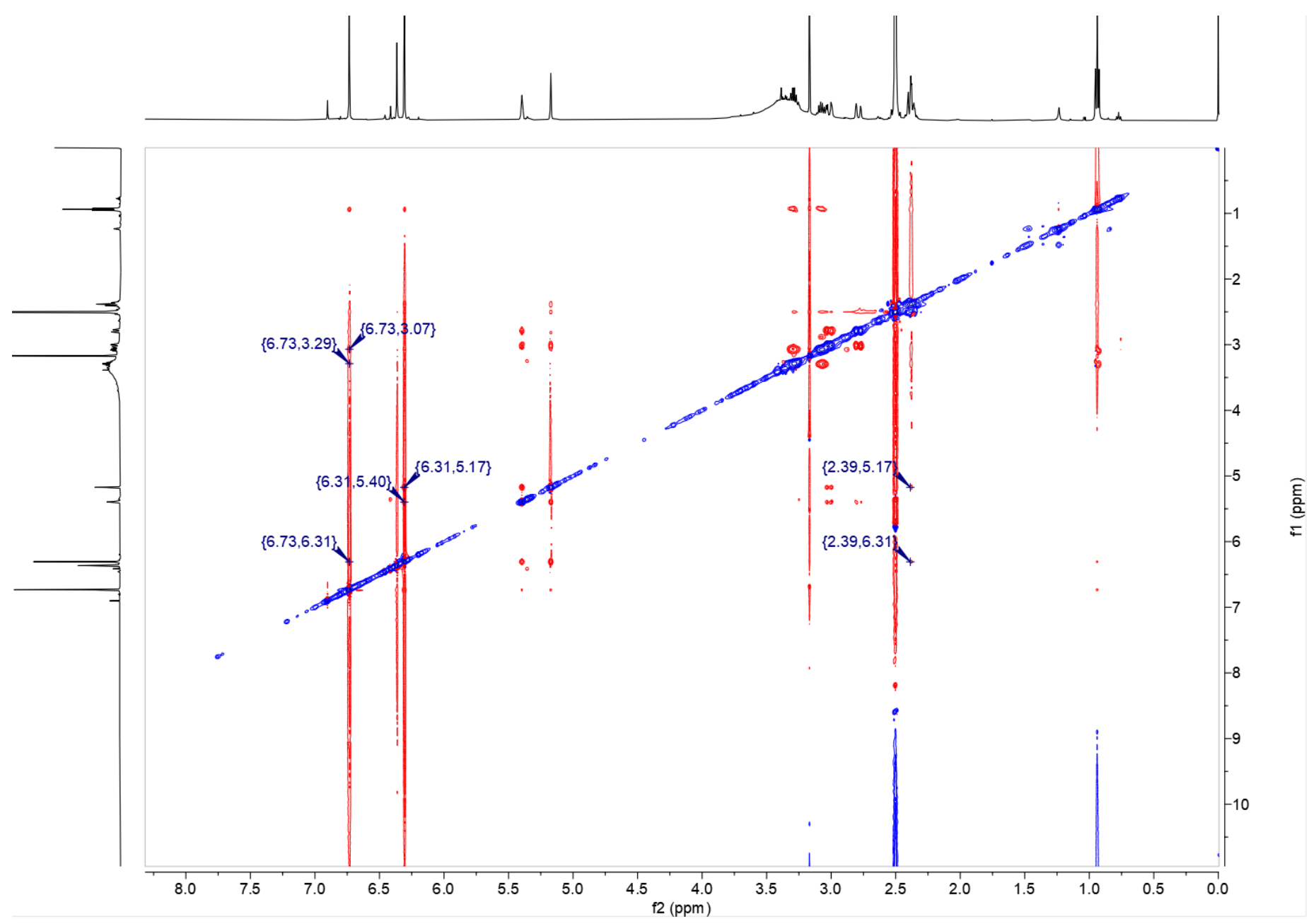


Figure S22. DEPT-135 spectra of compound 2 in DMSO- $d_{6}$.

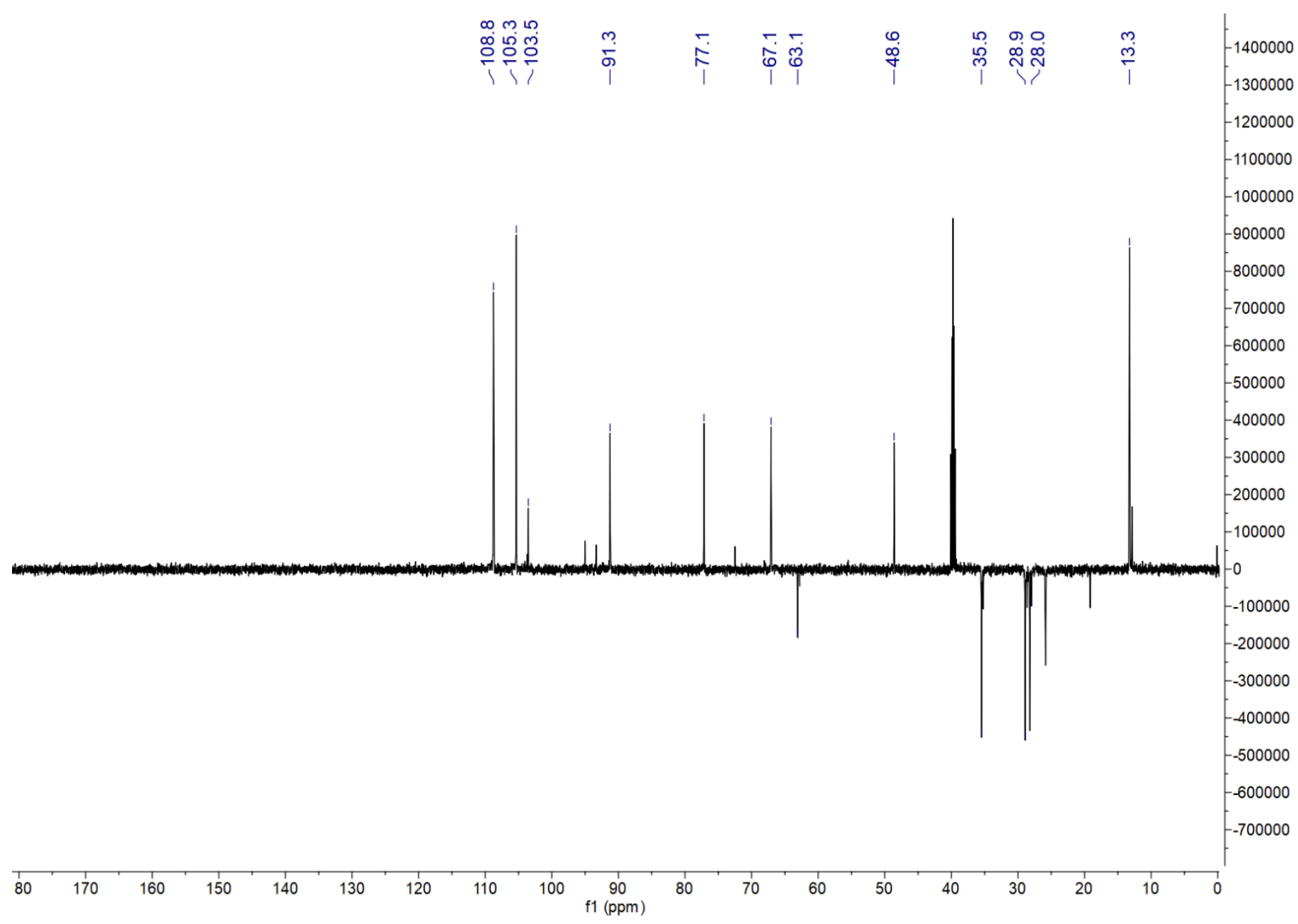


Figure S23. IR spectrum of compound 3.

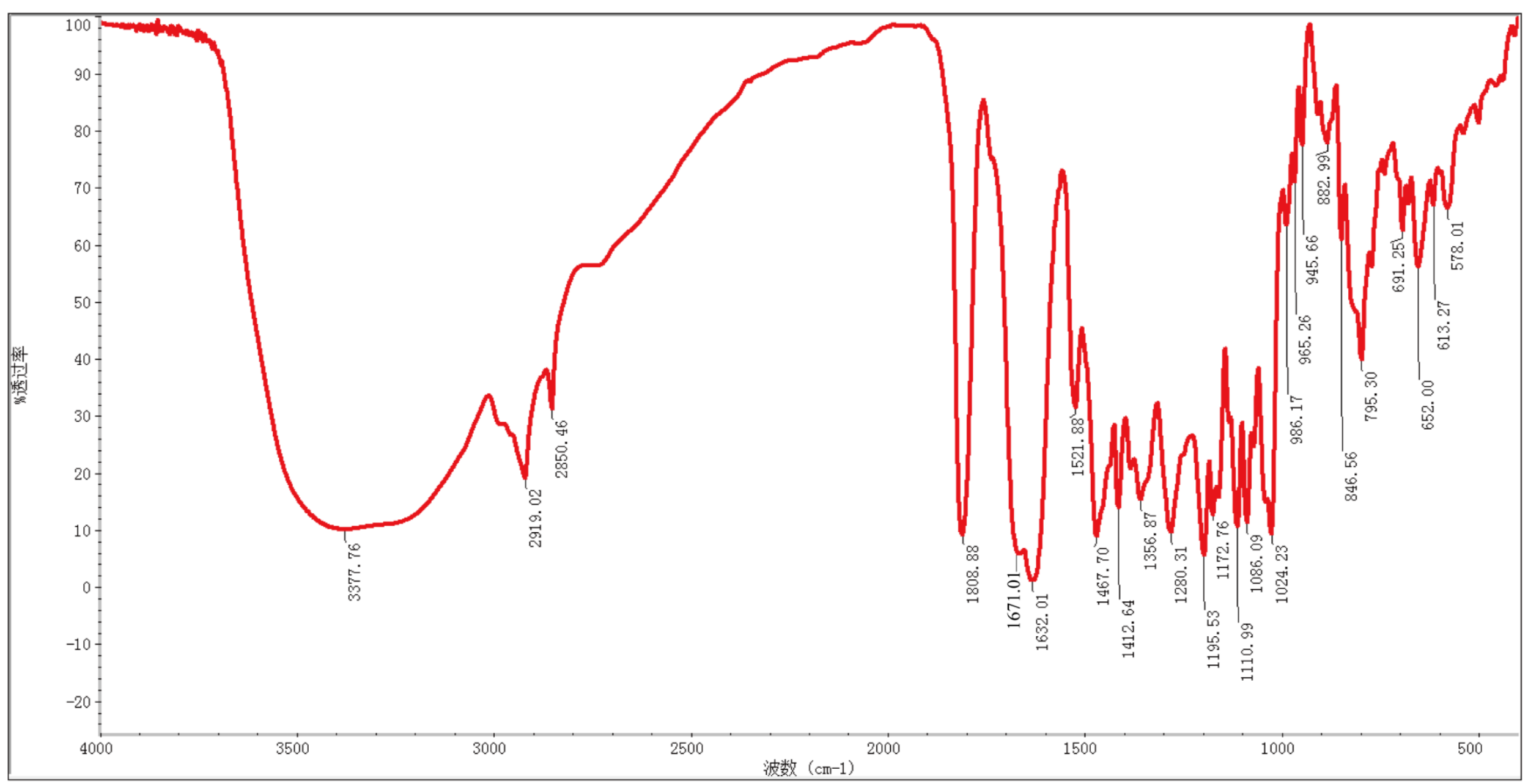


Figure S24. ${ }^{1} \mathrm{H}$ NMR spectra of compound 3 in DMSO- $d_{6}$.

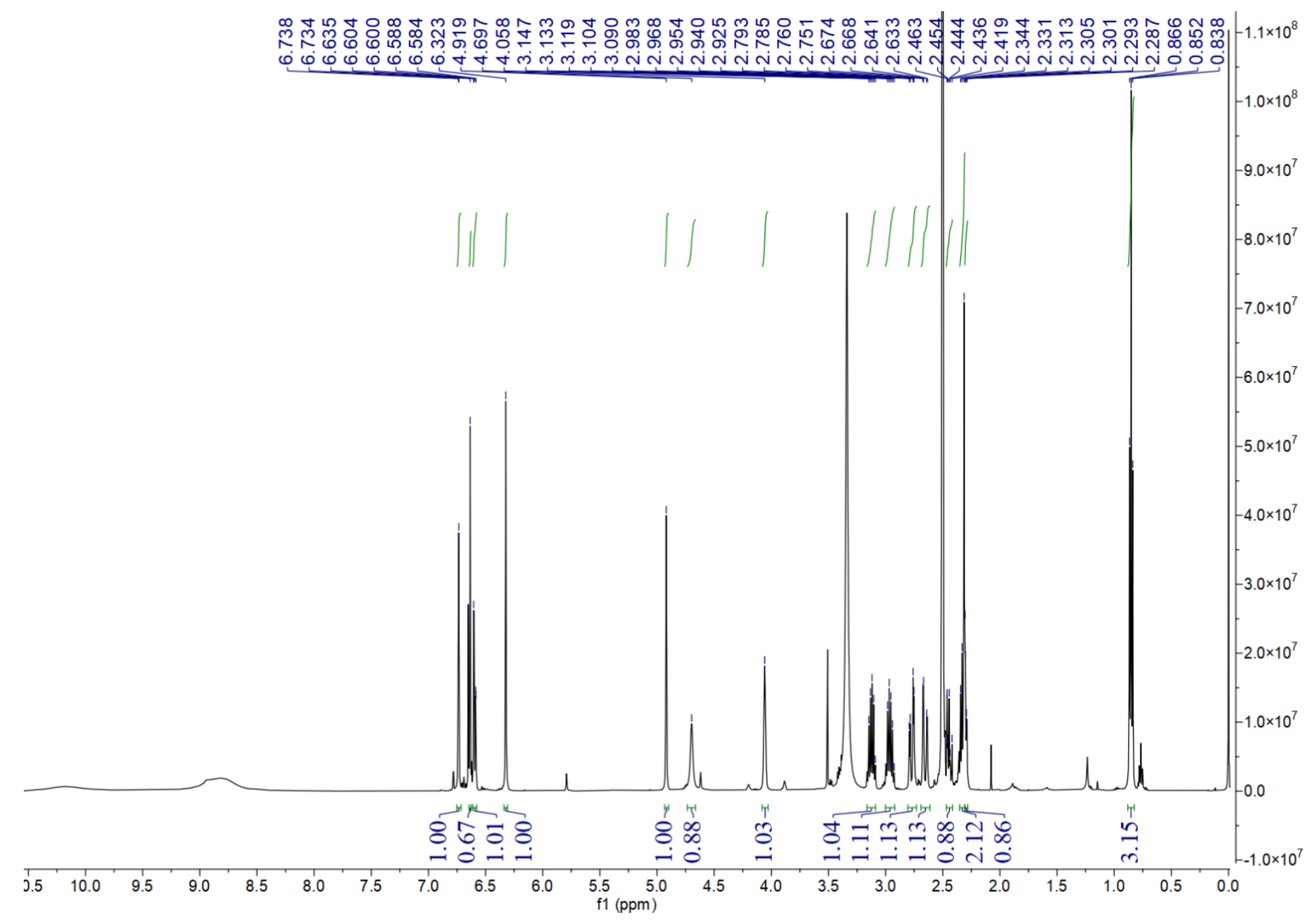


Figure S25. ${ }^{13} \mathrm{C}$ NMR spectra of compound 3 in DMSO- $d_{6}$.

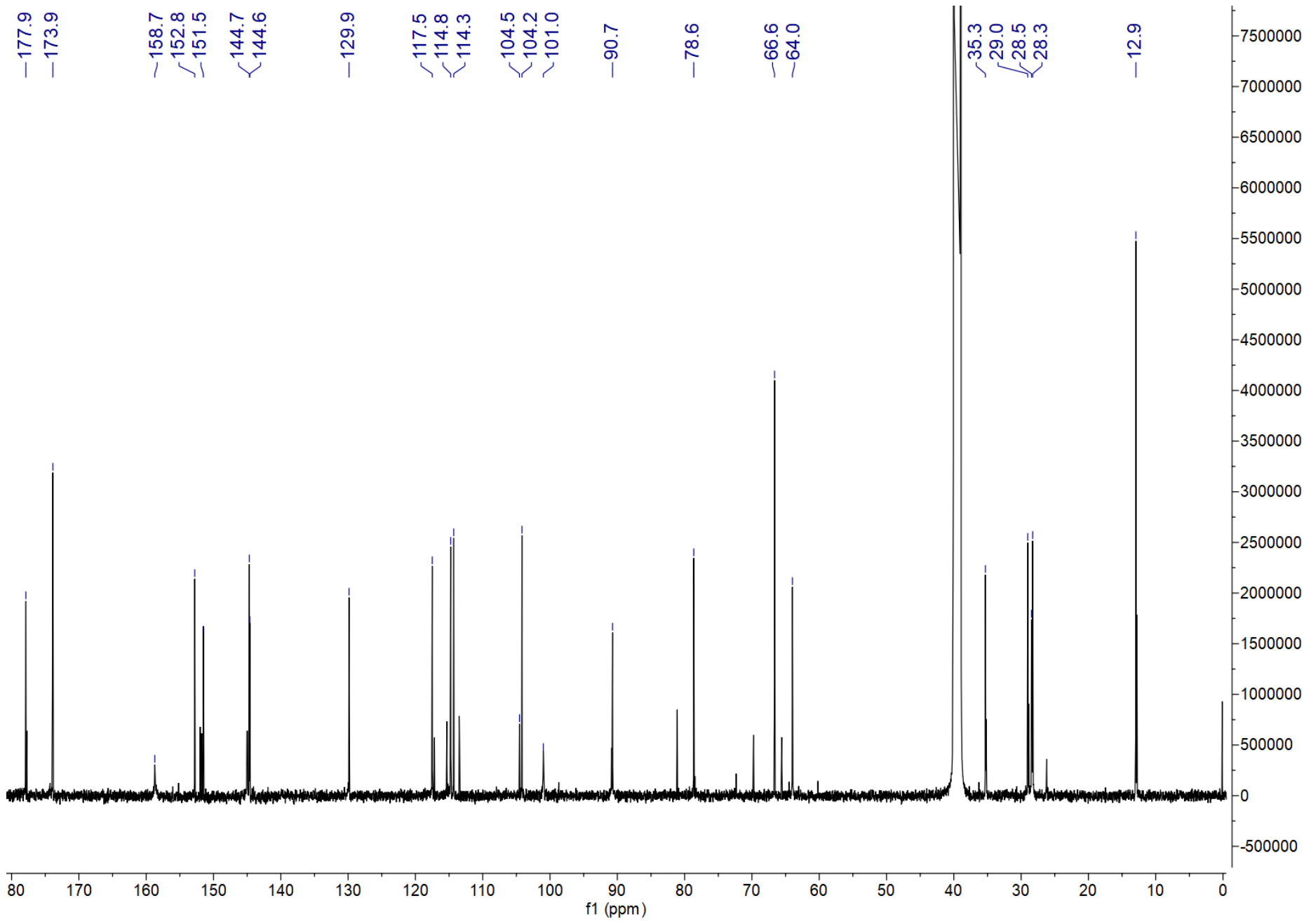


Figure S26. HSQC spectra of compound 3 in DMSO- $d_{6}$.
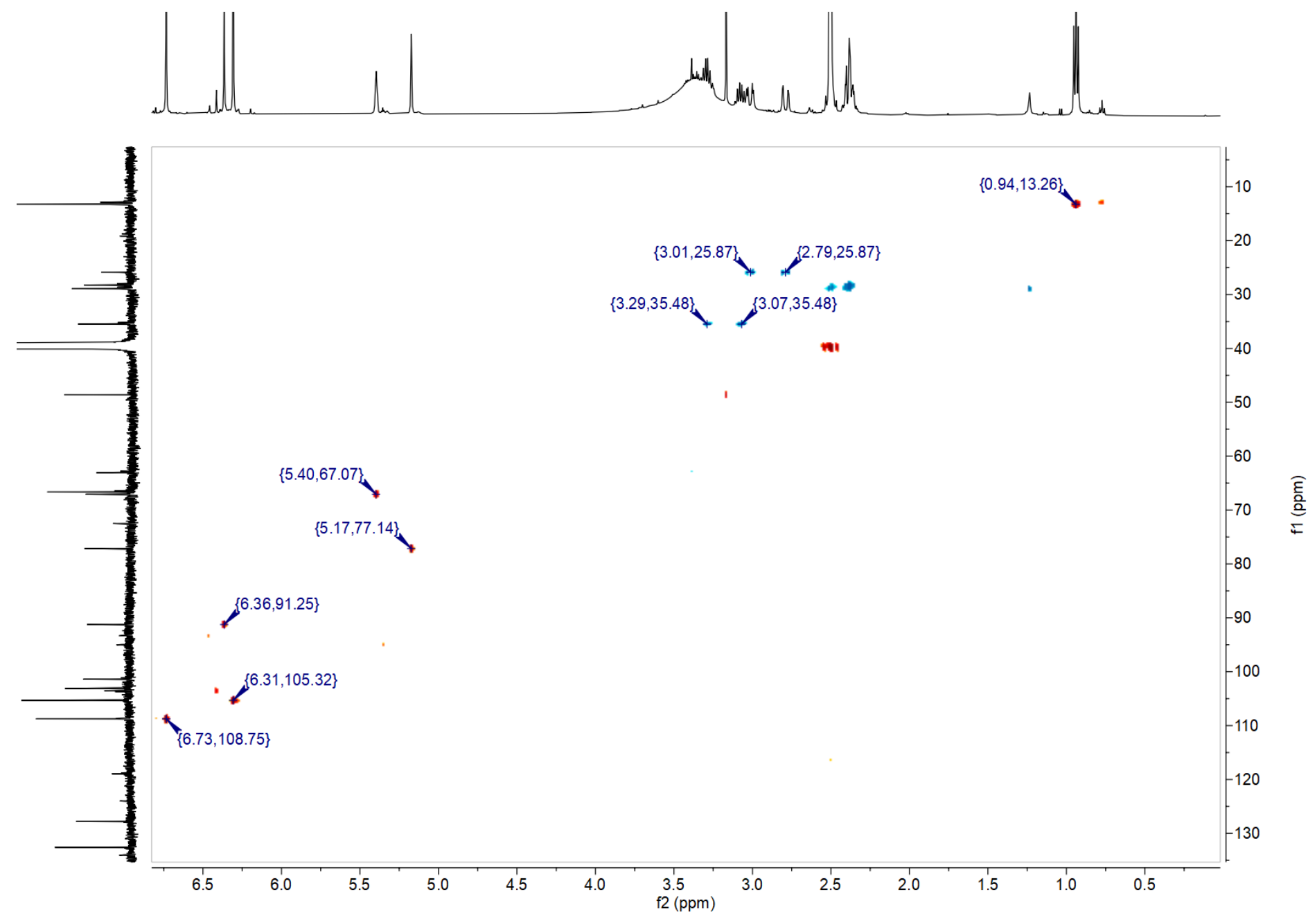


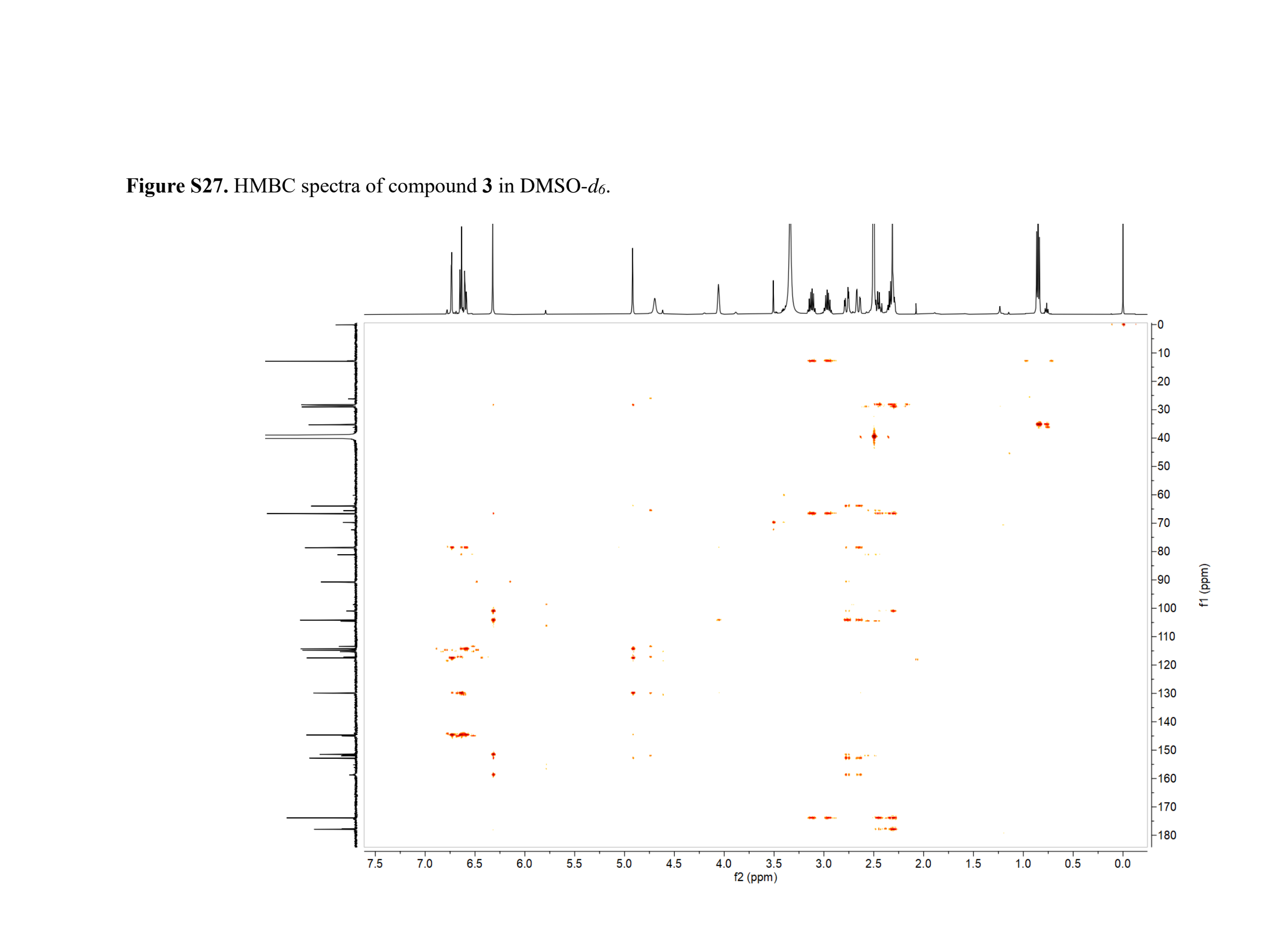


Figure S28. COSY spectra of compound 3 in DMSO- $d_{6}$.

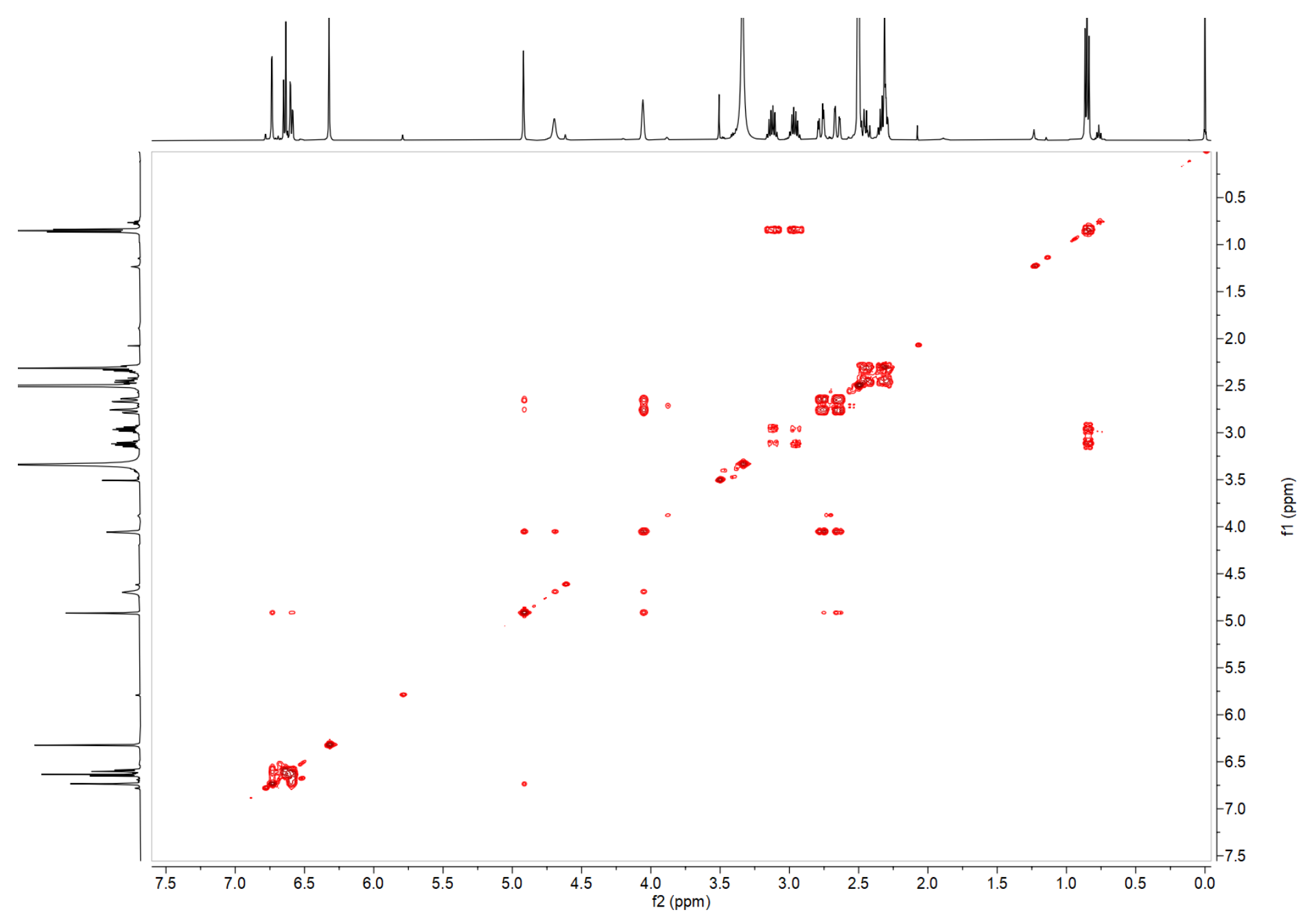


Figure S29. NOESY spectra of compound 3 in DMSO- $d_{6}$.

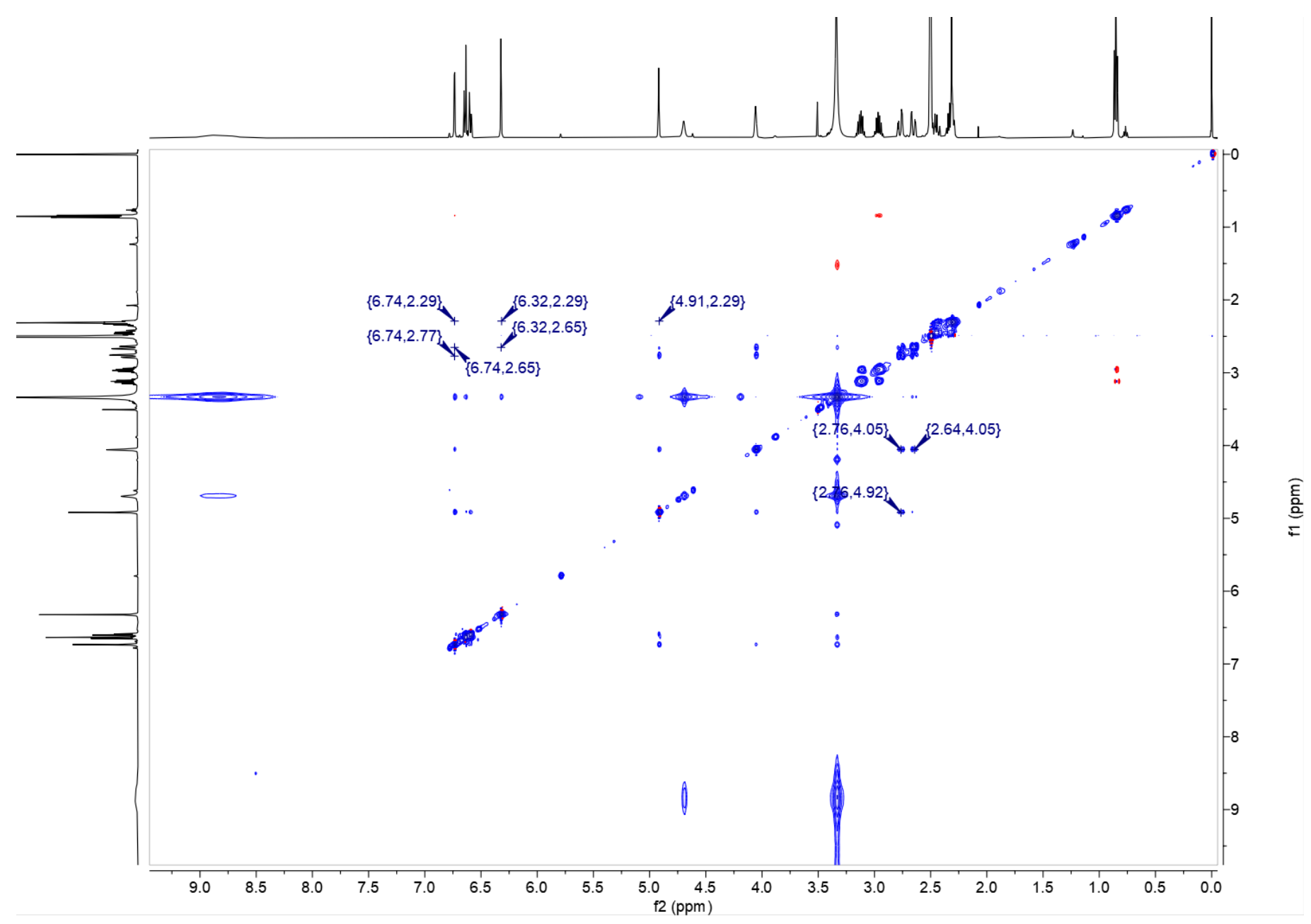


Figure S30. DEPT-135 spectra of compound 3 in DMSO- $d_{6}$.

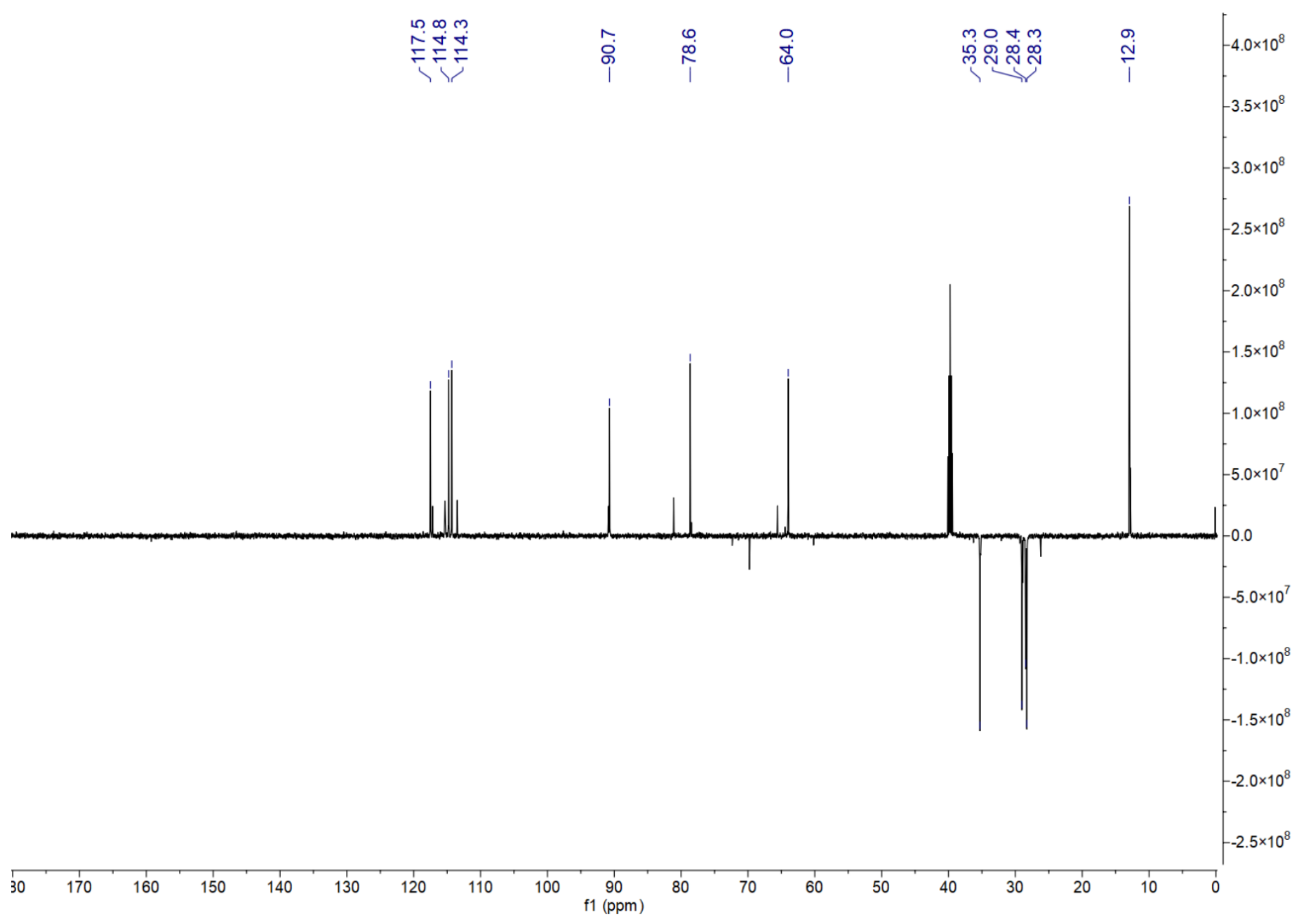


Figure S31. Local NOESY spectra of compound 3 in DMSO- $d_{6}$.

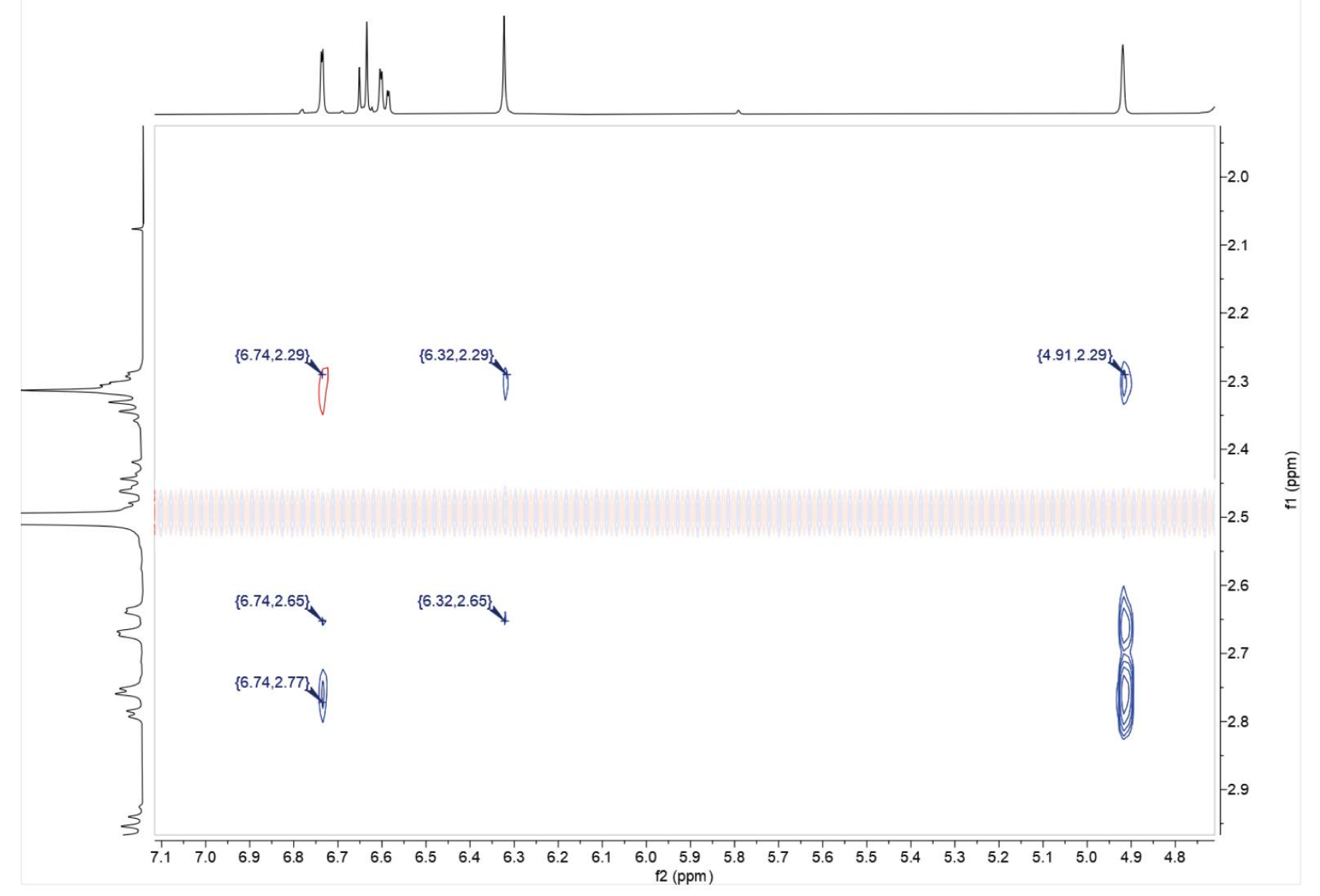


Figure S32. IR spectrum of compound 4.

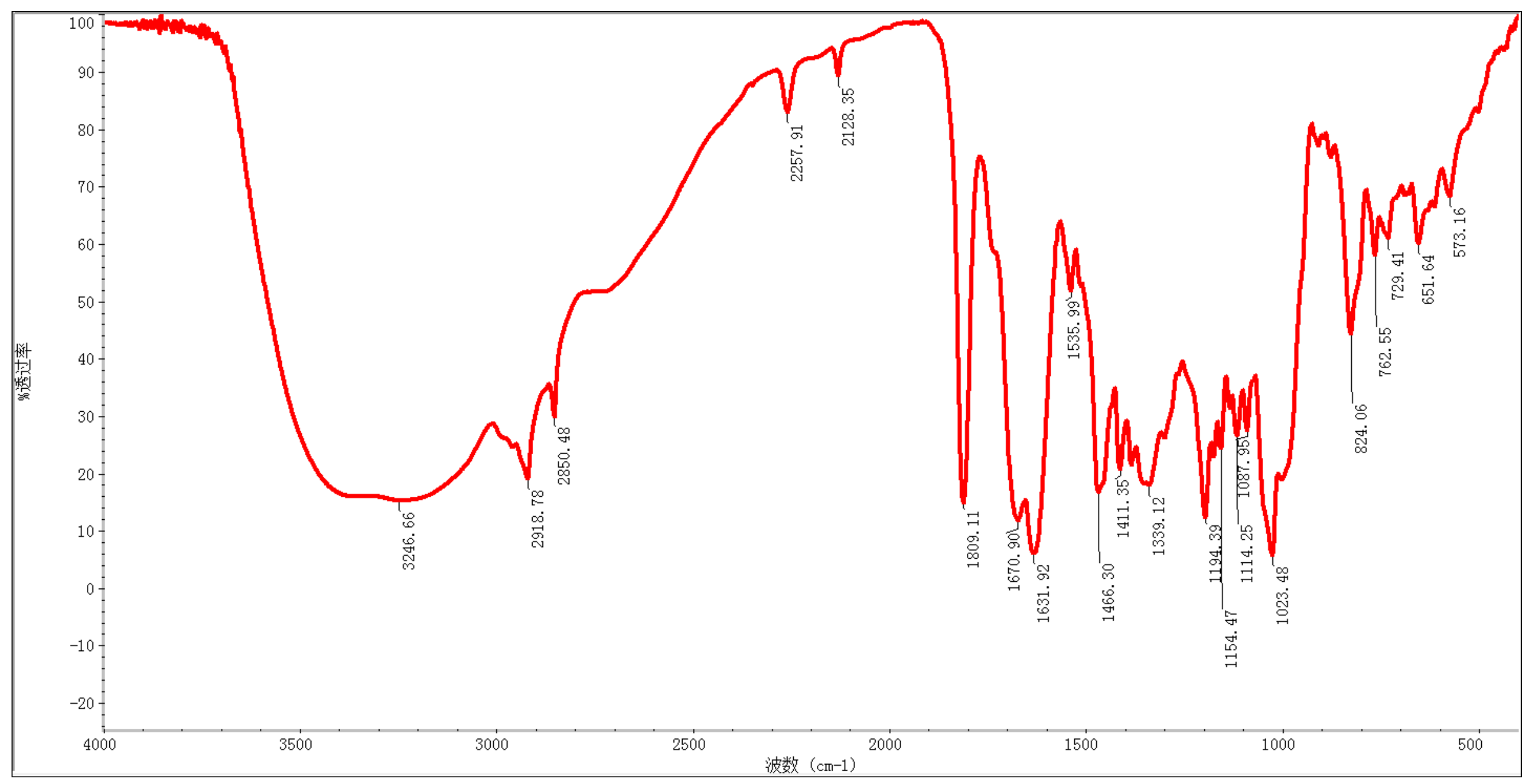


Figure S33. ${ }^{1} \mathrm{H}$ NMR spectra of compound 4 in DMSO- $d_{6}$.

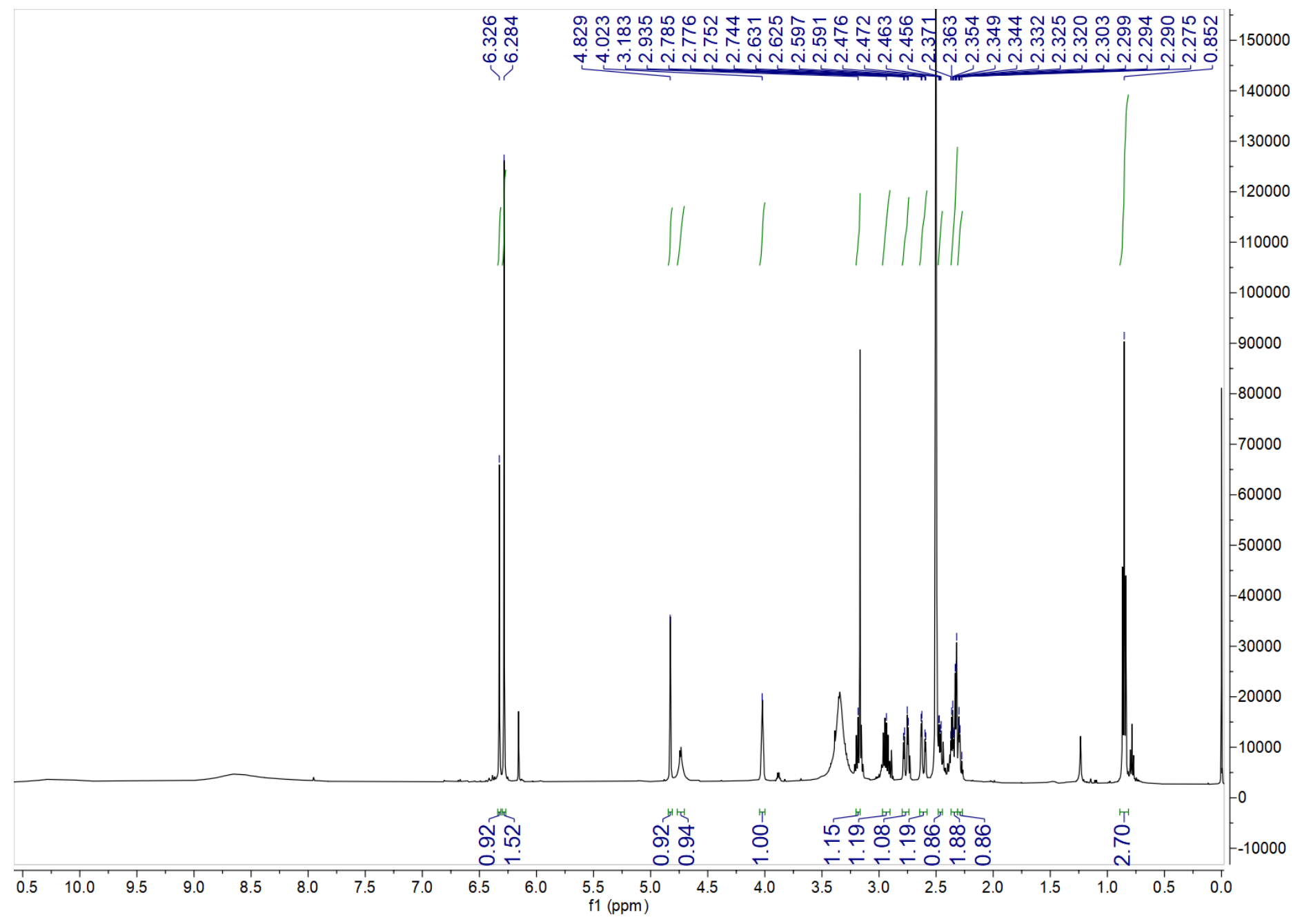


Figure S34. ${ }^{13} \mathrm{C}$ NMR spectra of compound 4 in DMSO- $d_{6}$.

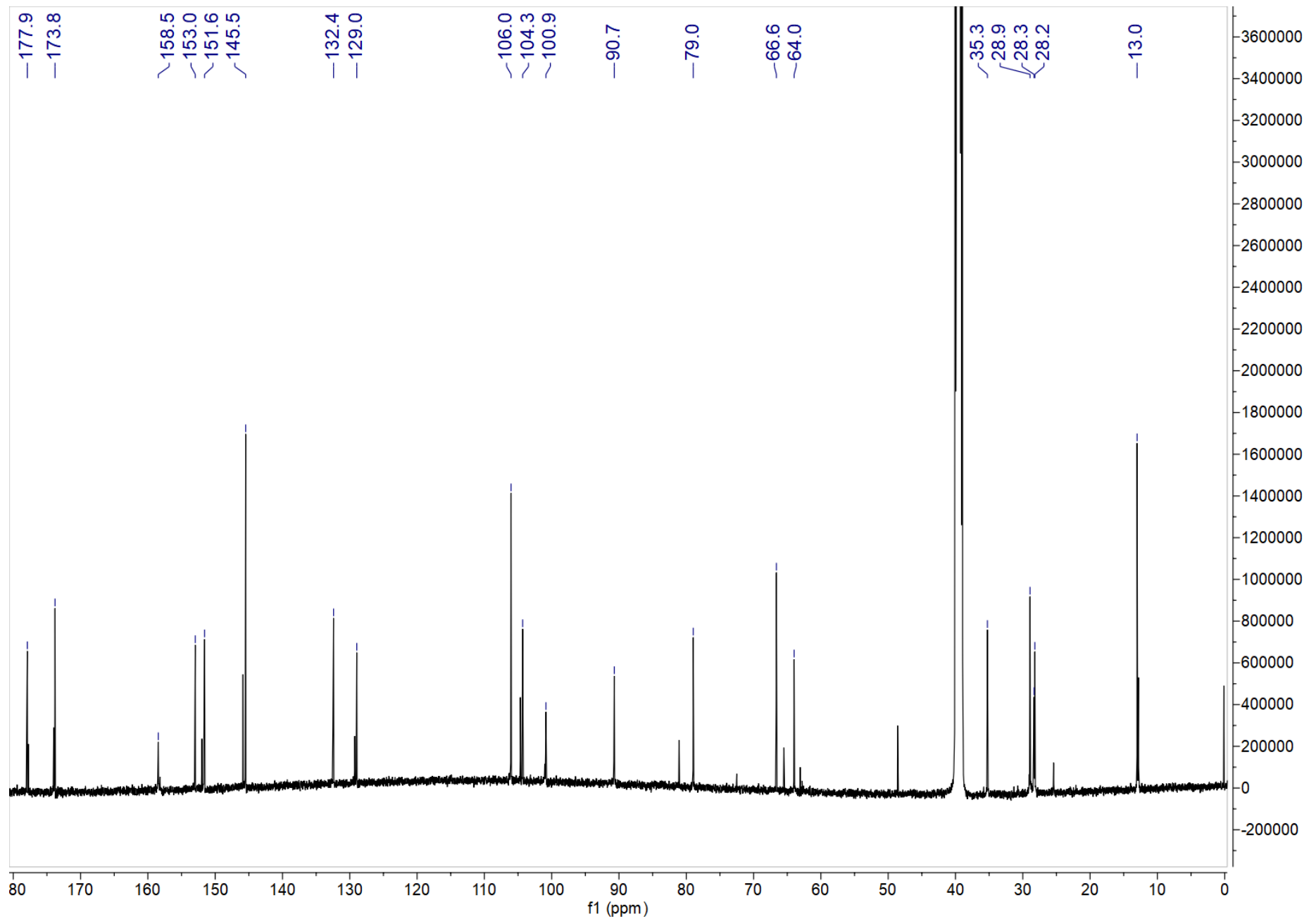


Figure S35. HSQC spectra of compound 4 in DMSO- $d_{6}$.

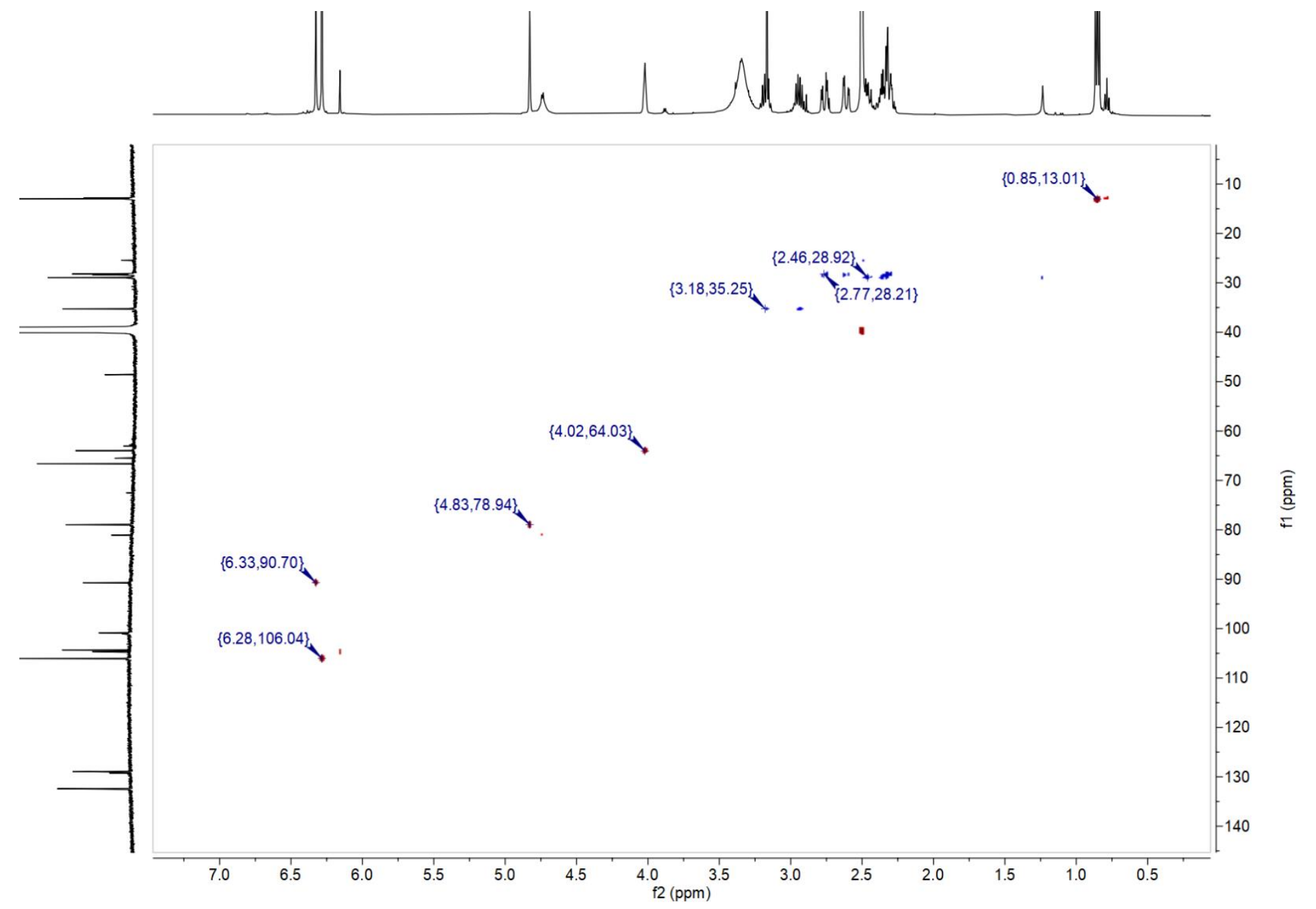


Figure S36. HMBC spectra of compound 4 in DMSO- $d_{6}$.

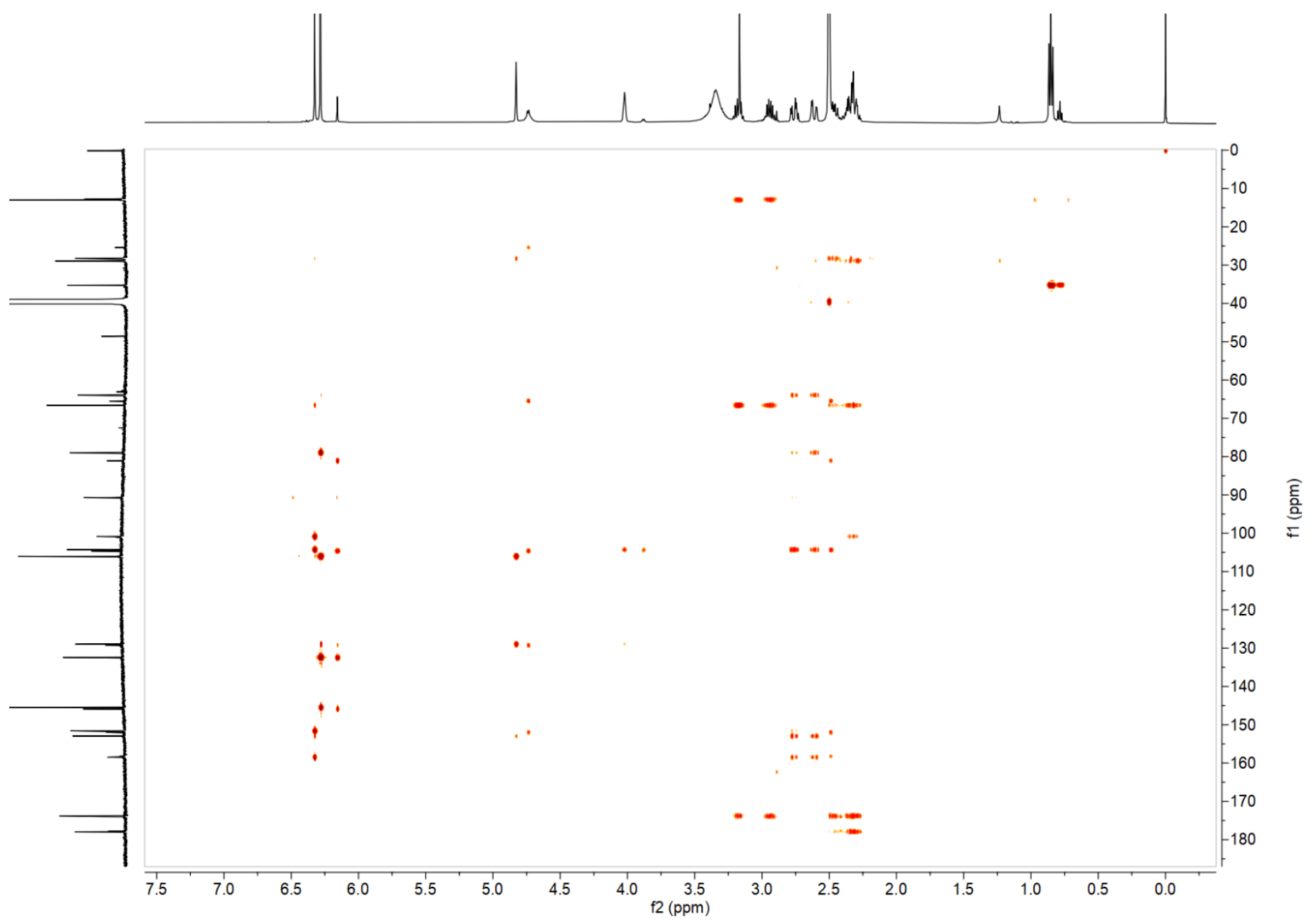


Figure S37. COSY spectra of compound 4 in DMSO- $d_{6}$.

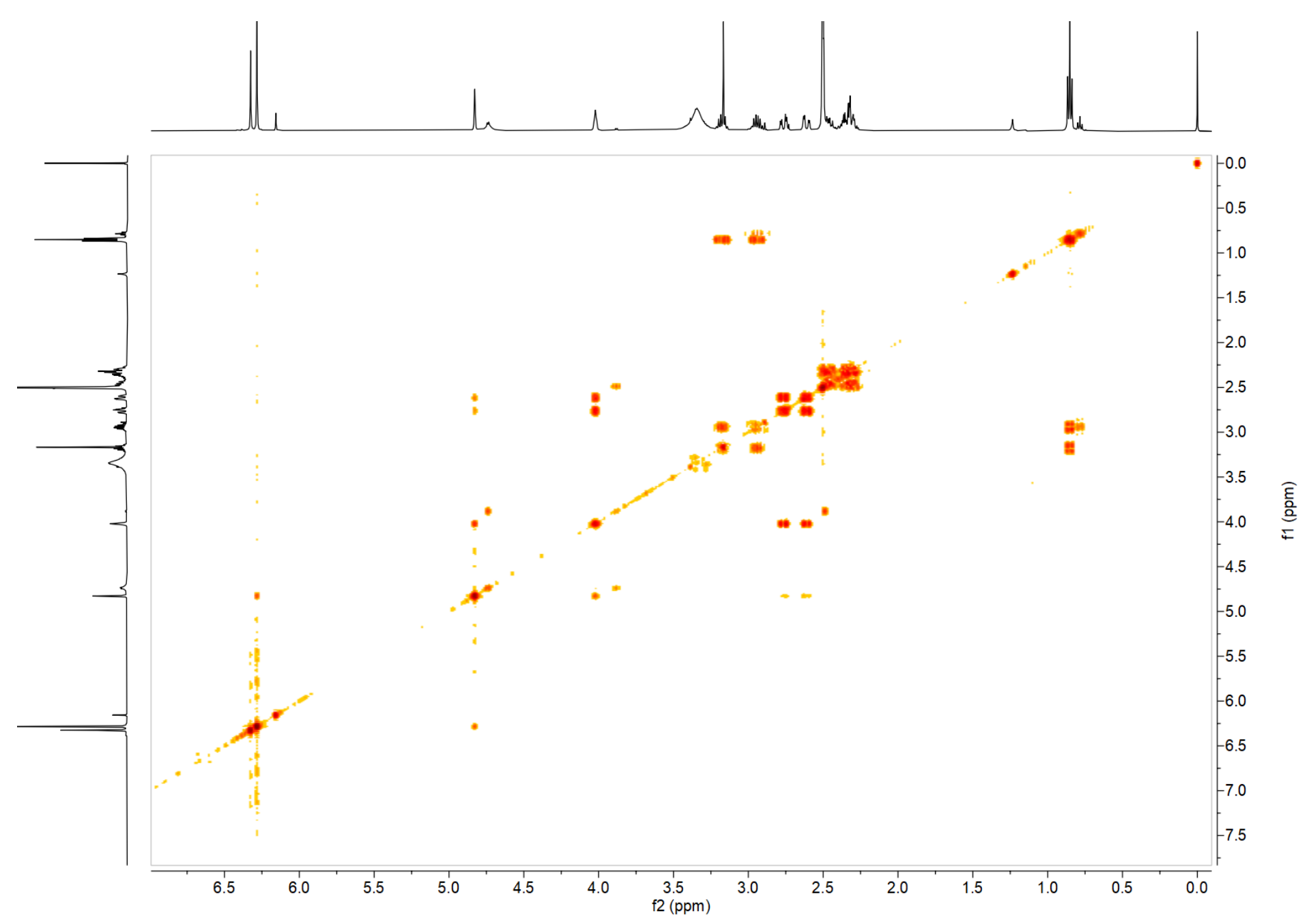




$$
\overline{z^{3}}
$$


Figure S39. DEPT-135 spectra of compound 4 in DMSO- $d_{6}$.

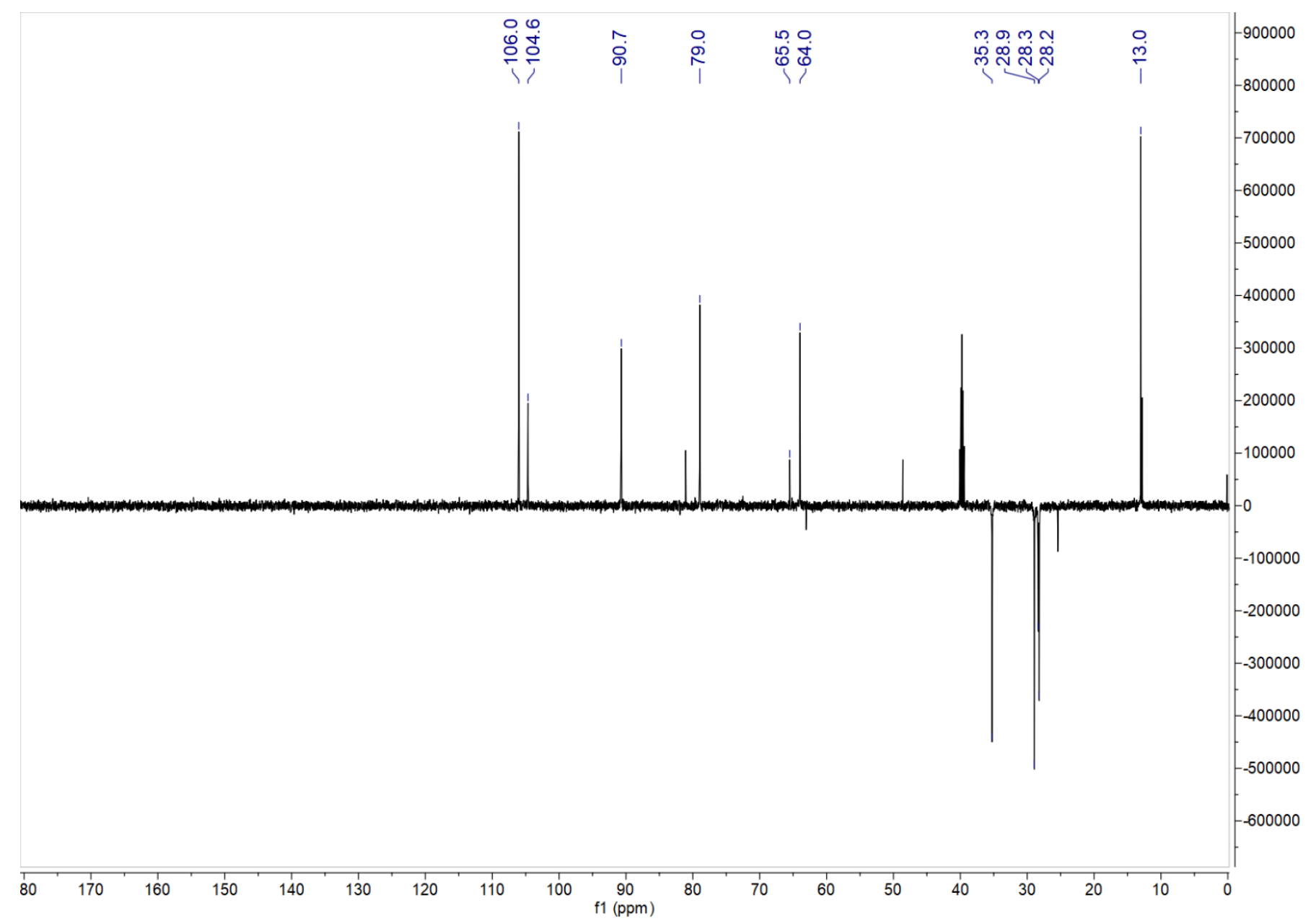


Figure S40. MS/MS spectrum of compound 1, 2, 3 and 4.
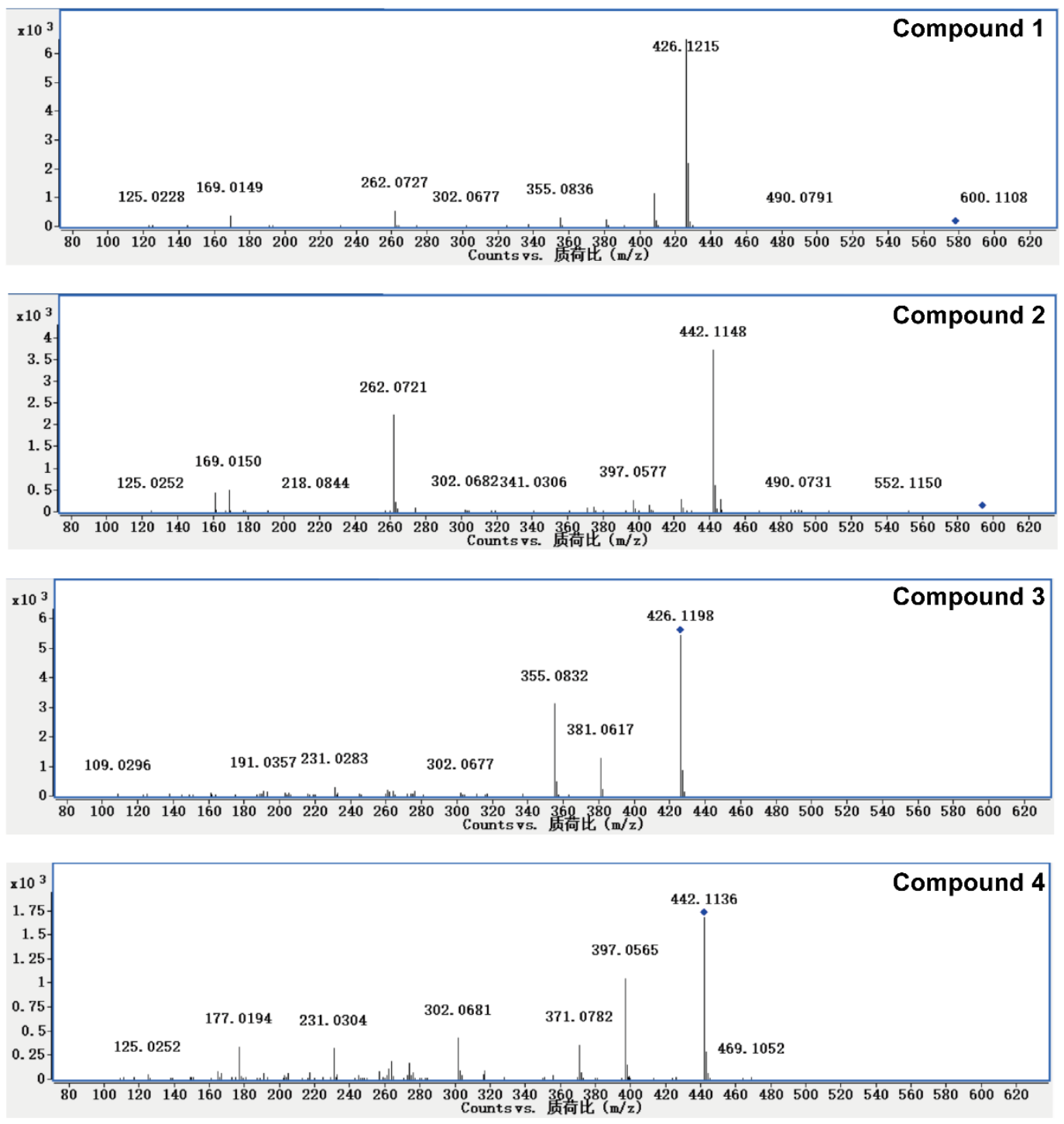
Figure S41. 3D molecular model of compounds 1,2, 3, and $\mathbf{4}$ showing key NOESY (dashed double arrowhead line) correlations.
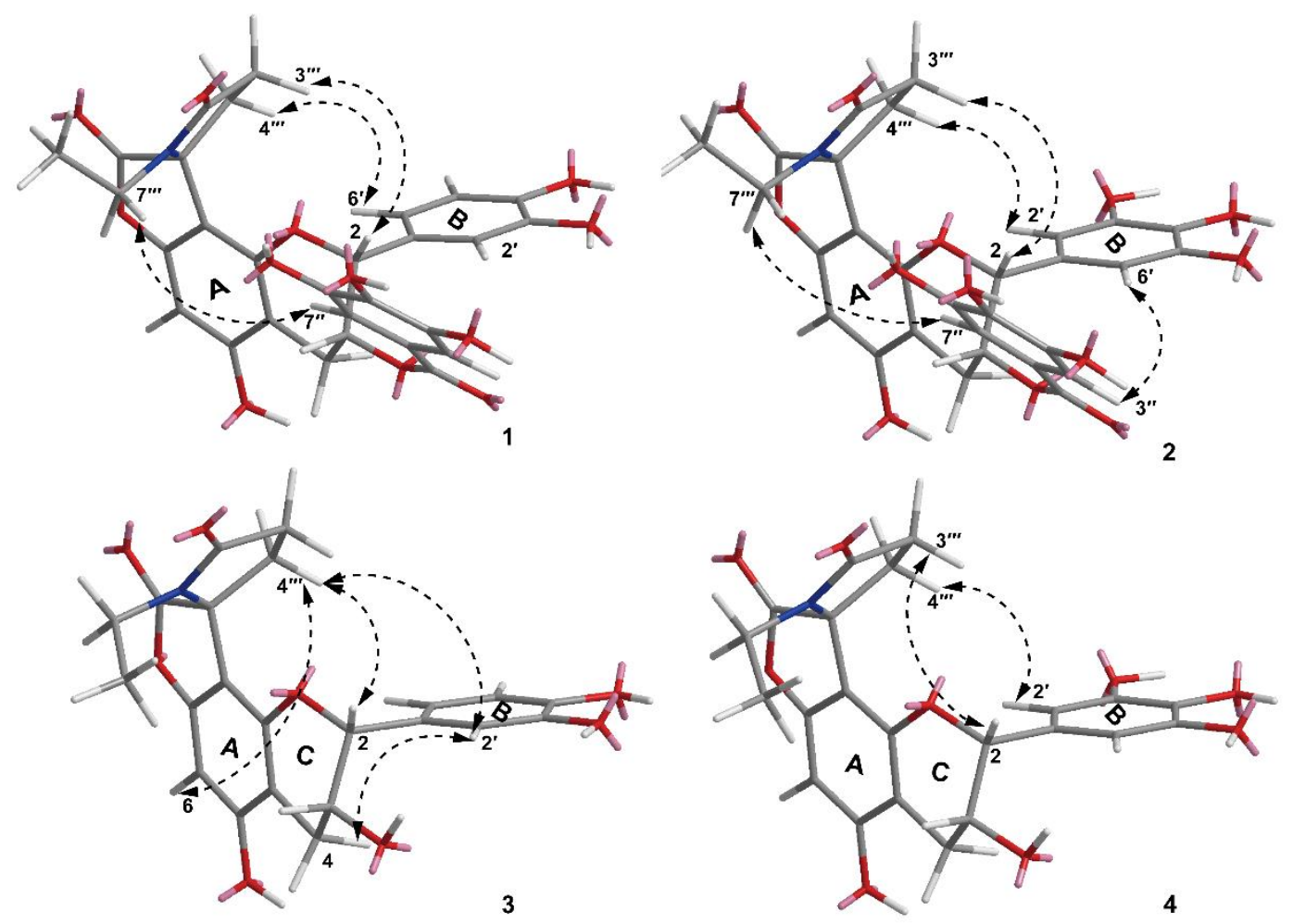
Figure S42. Key NOESY correlations between $\mathrm{H}-2$ and $\mathrm{H}_{2}-4$ for compounds 1-4.

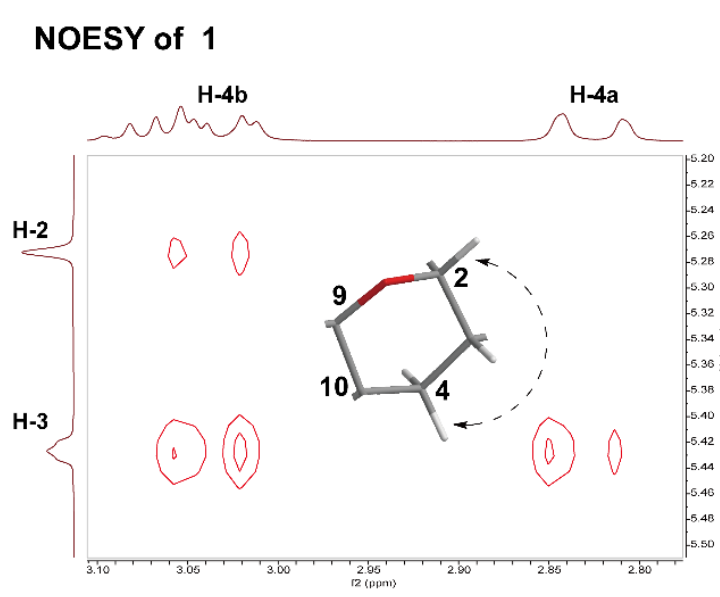

NOESY of 3

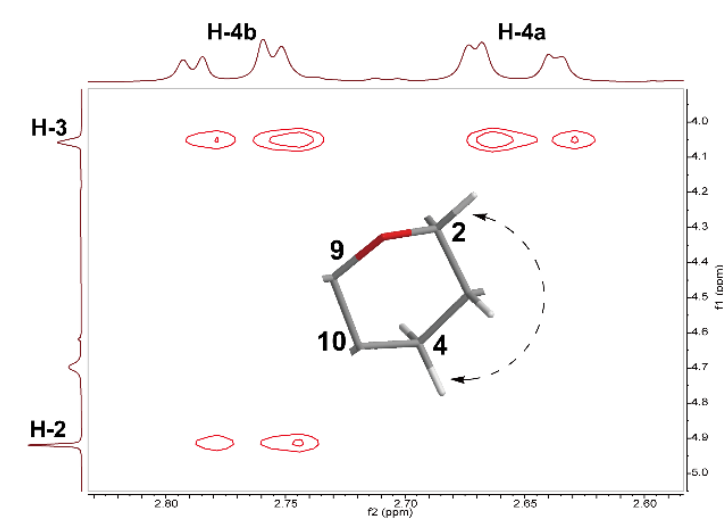

\section{NOESY of 2}

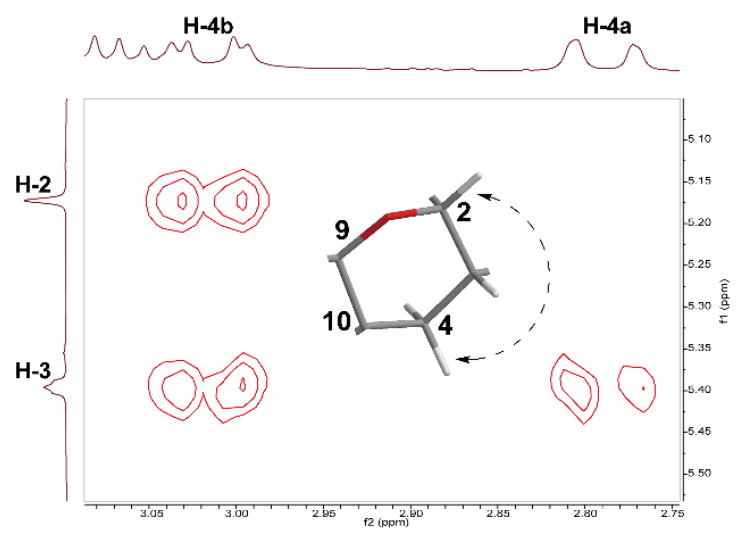

NOESY of 4

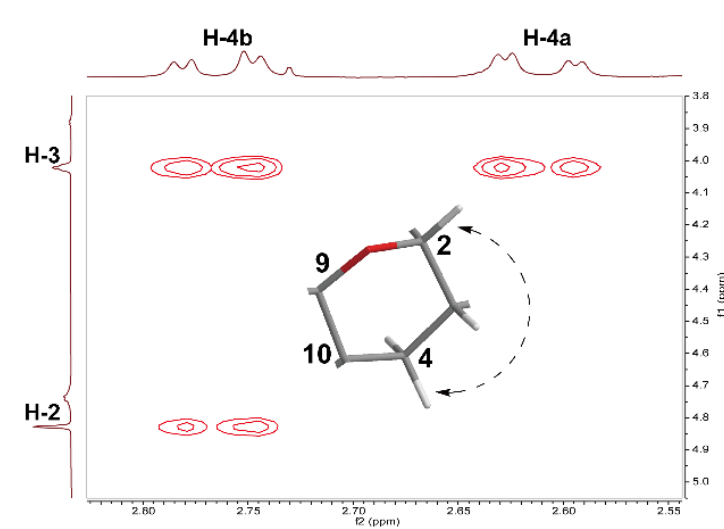


Figure S43. Local ${ }^{1} \mathrm{H}$ NMR spectra of compound 3 before (brown line) and after (green line) the storage in DMSO- $d_{6}$.

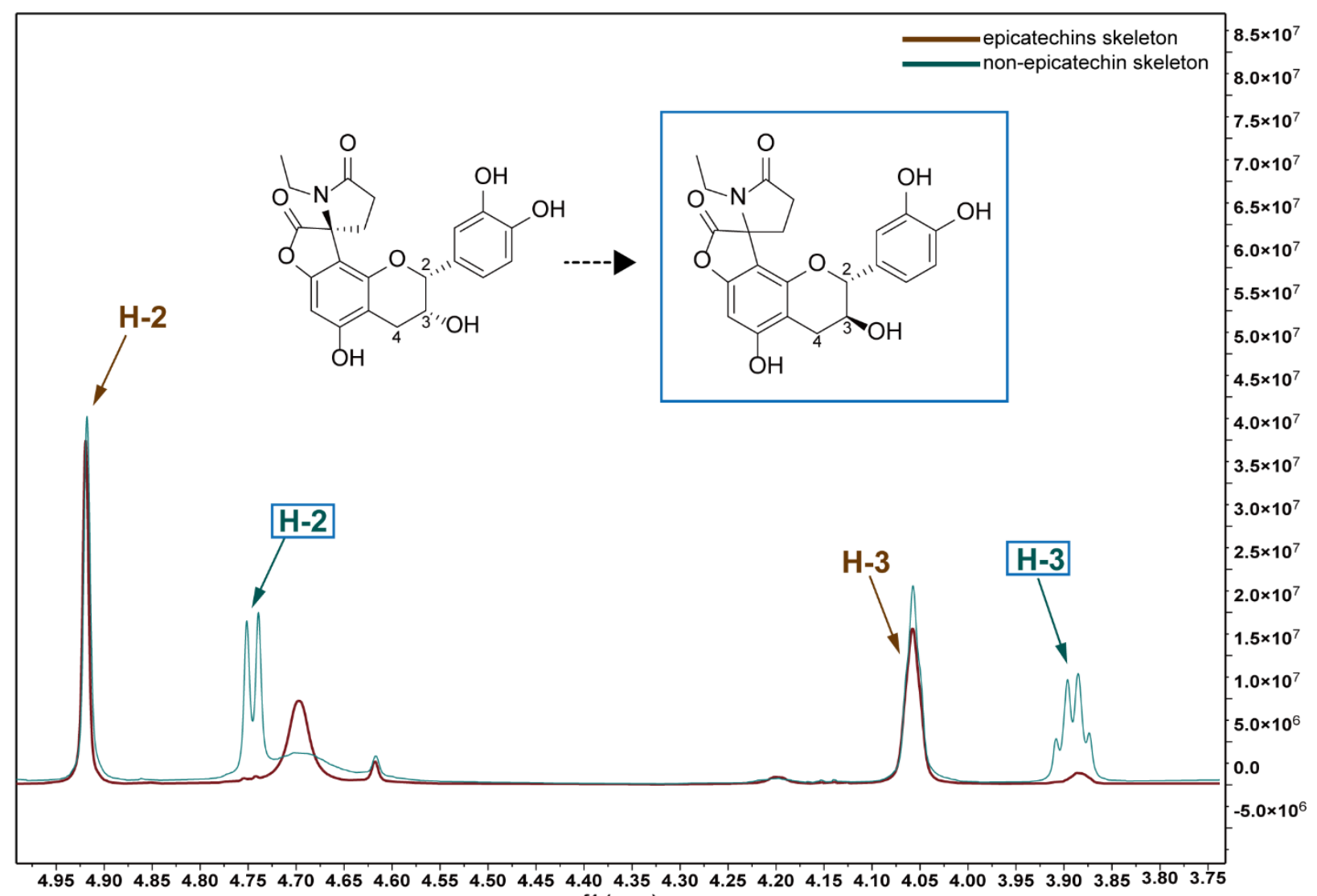

$\mathrm{f1}(\mathrm{ppm})$ 
Figure S44. (A) TIC of the 70\% aqueous MeOH extract of YDGT fresh leaves compared with the TIC of compounds 1, 2, 3 and 4. (B) HPLC chromatogram of the $70 \%$ aqueous $\mathrm{MeOH}$ extract of YDGT fresh leaves compared with the HPLC chromatogram of compounds $\mathbf{1 , 2 , 3}$ and $\mathbf{4}$.
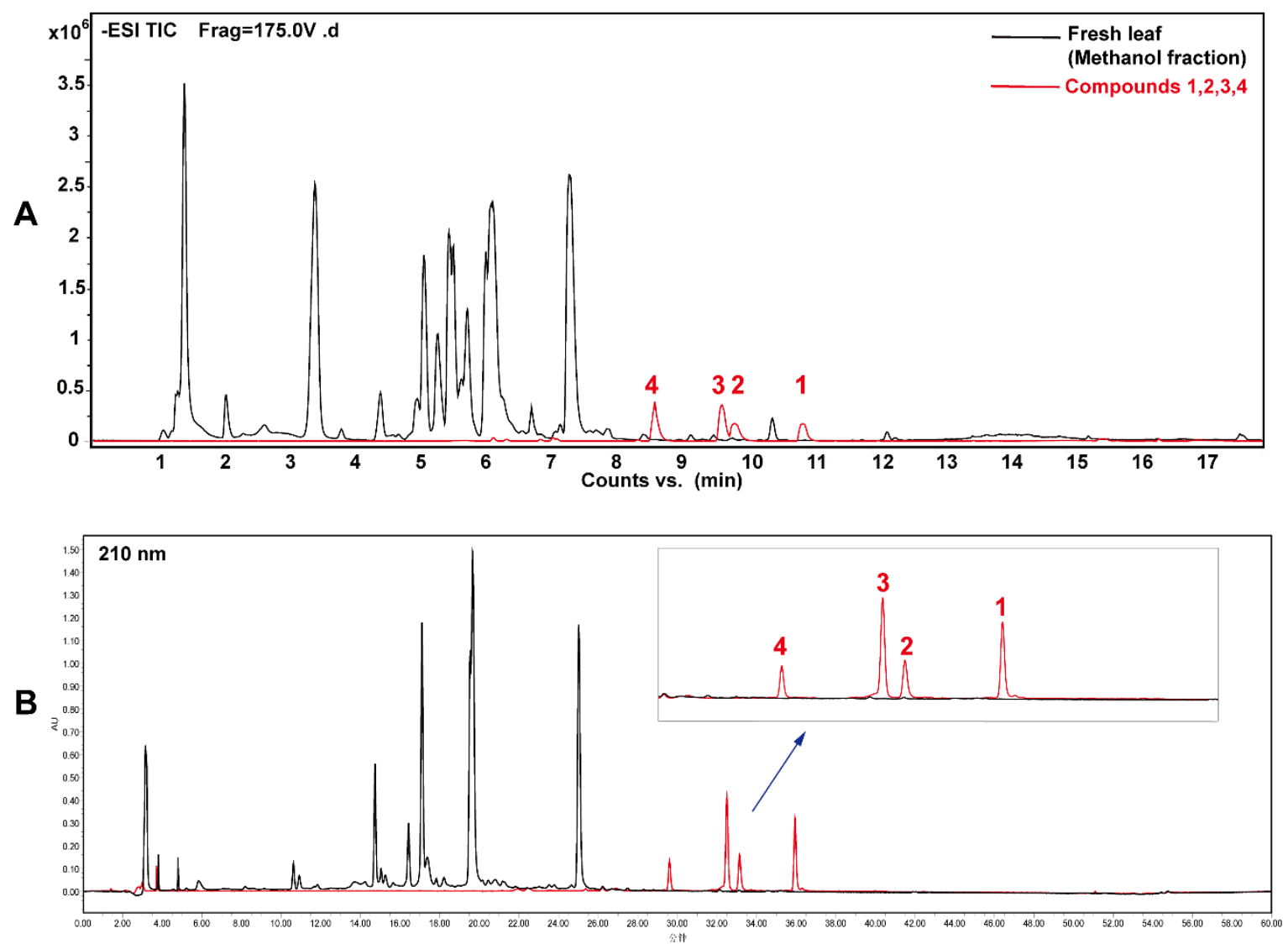
Figure S45. TIC of flavoalkaloids ([M-H] $]^{-}$568.14, $\left.[\mathrm{M}-\mathrm{H}]^{-} 552.15\right)$ and compounds $\mathbf{1 , 2 , 3}$ and 4.

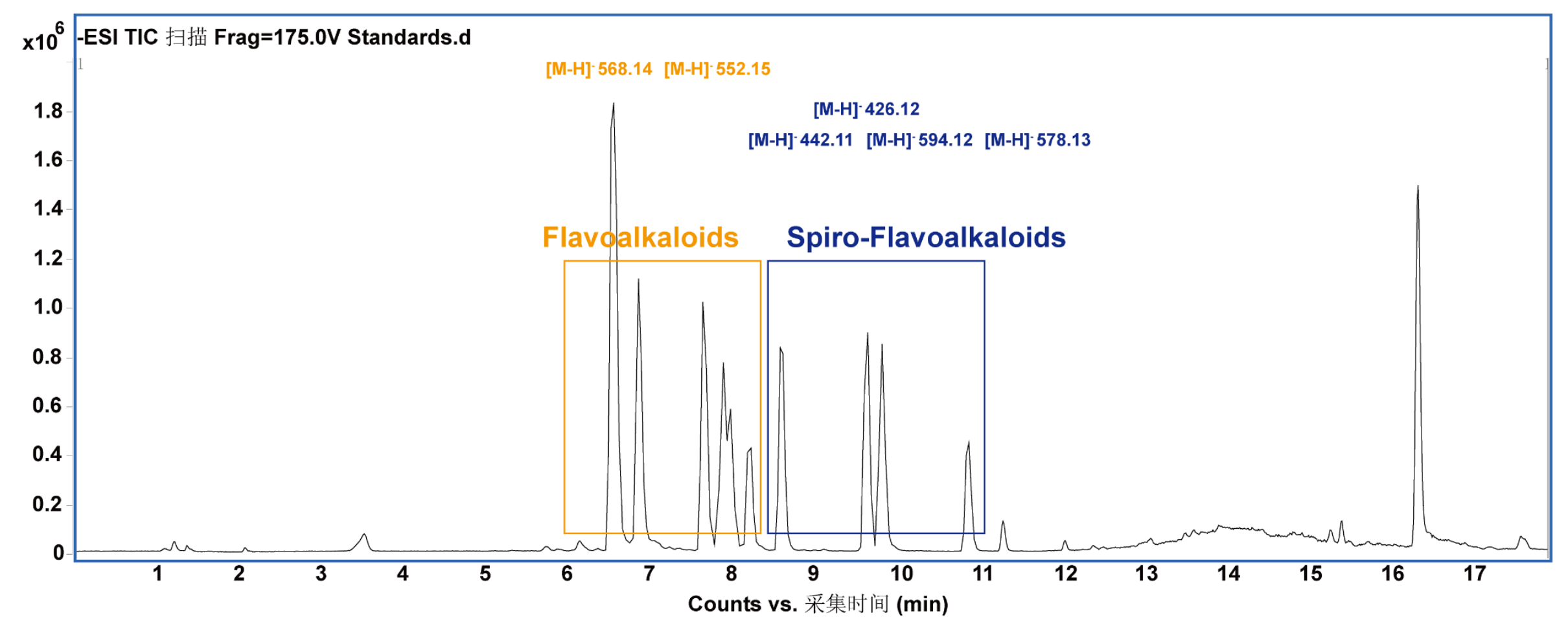


Figure S46. TIC of the MeOH fractions of green tea (Camellia sinensis var. assamica.) after extraction with PE and EtOAc.

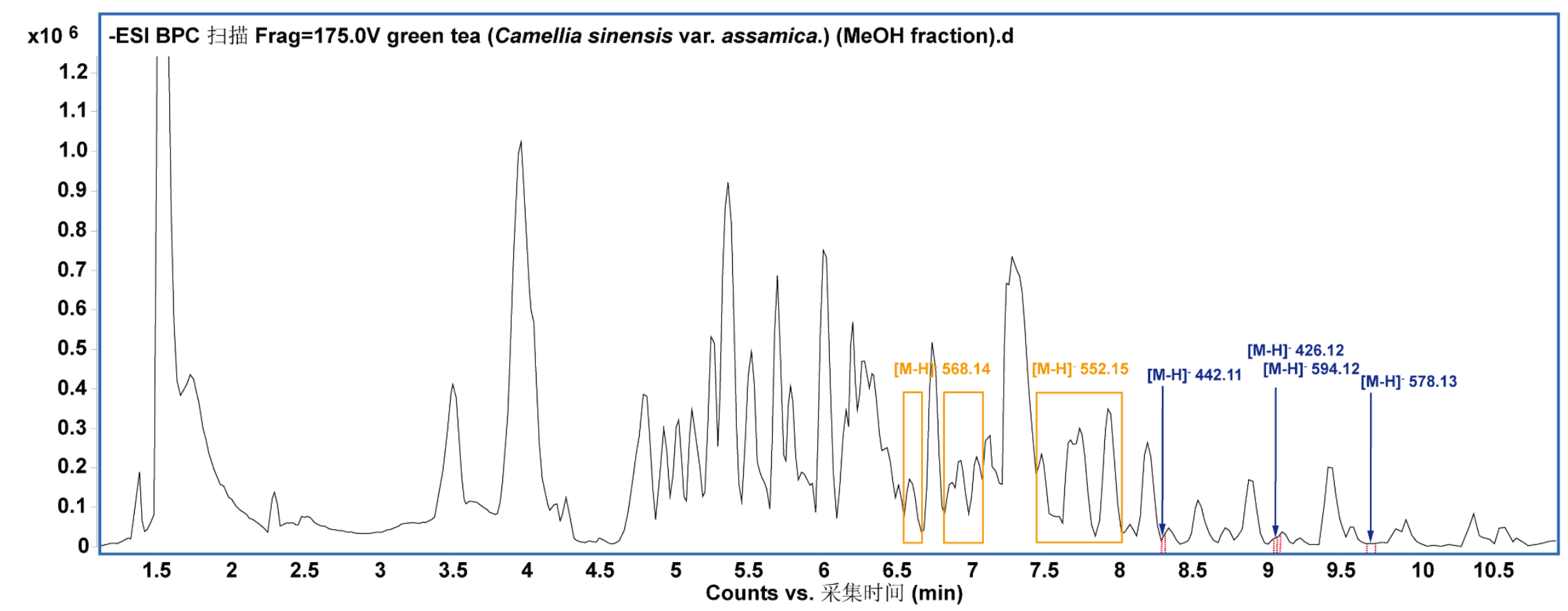


Figure S47. Possible formation route of spiro-flavoalkaloids in tea processing.

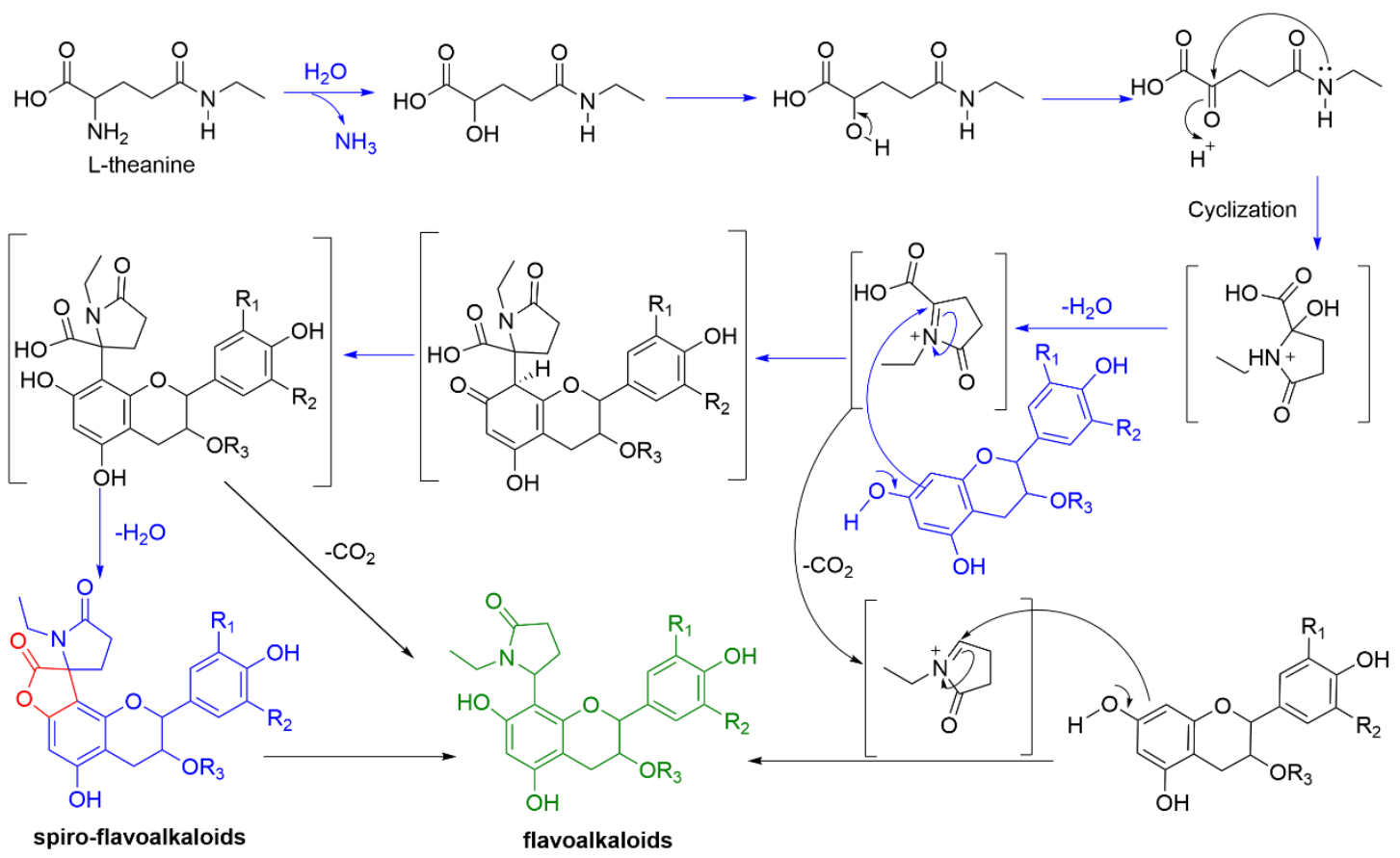

Figure S48. Interactions of acarbose with $\alpha$-glucosidase and the 3D structure of $\alpha$ glucosidase-acarbose complex with the key residues.

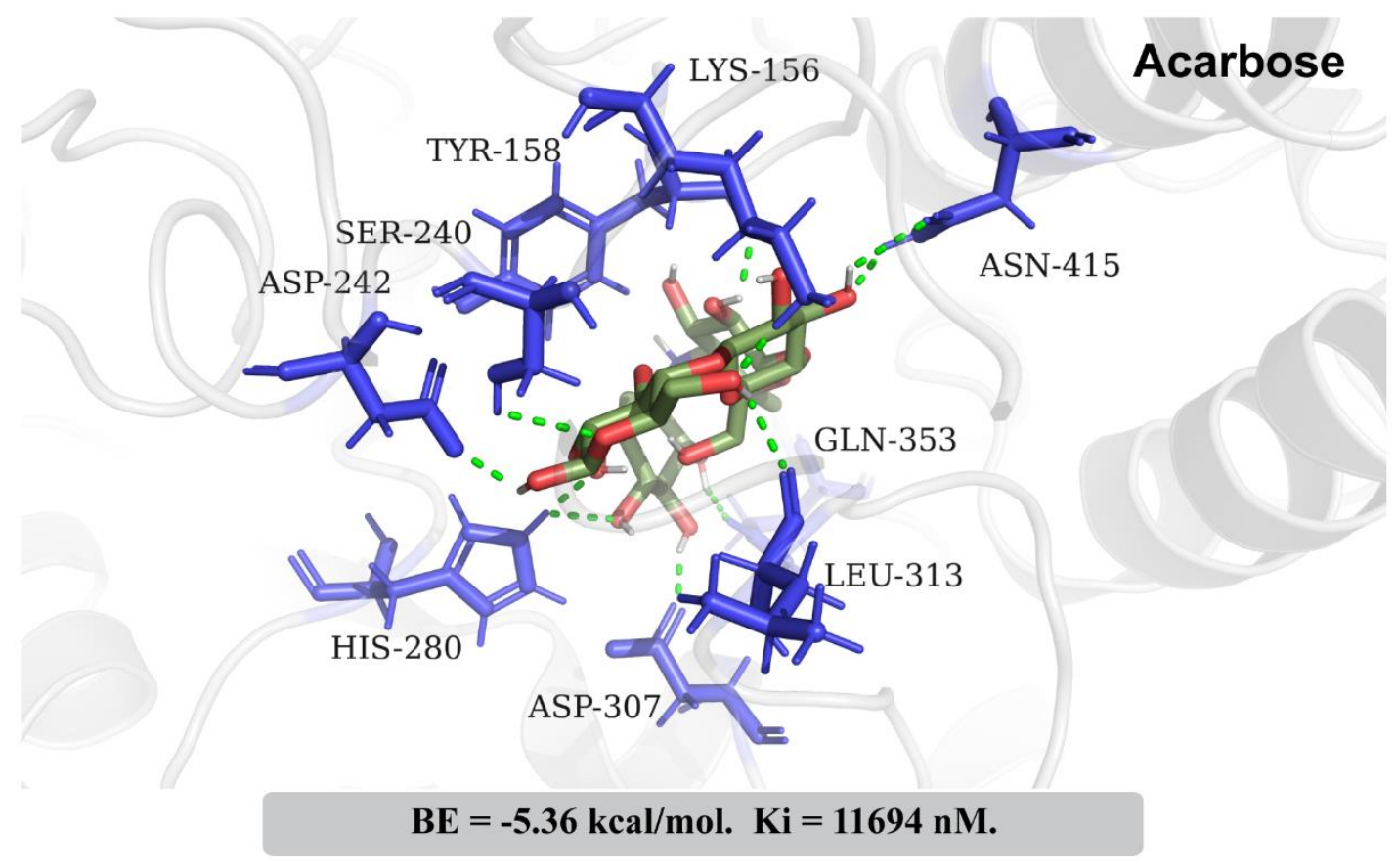




\section{The detailed information of fluorescence quenching experiment.}

Fluorescence quenching experiment of $\alpha$-glucosidase. Cary eclipse fluorescence spectrophotometer (Agilent Inc., Santa Clara, CA) was used to determine $\alpha$-glucosidase fluorescence spectra alone and $\alpha$-glucosidase with a series of concentrations of compounds $\mathbf{1}(0,10,20,30,40,50 \mu \mathrm{M})$ and $\mathbf{2}(0,10,20,30,40,50 \mu \mathrm{M})$, in a normal PBS buffer at $\mathrm{pH}$ 7.4. The flavoalkaloids were titrated at 293, 300 and $310 \mathrm{~K}$, respectively, while the $\alpha$-glucosidase concentration remained at $20 \mu \mathrm{M}$.

Type of fluorescence quenching of $\alpha$-glucosidase by compounds 1 and 2 . According to the fluctuation trends of excited state lifetime with temperature, the quenching types can be identified. Dynamic quenching of $\alpha$-glucosidase and compounds 1 and 2 was calculated by Stern-Volmer Eq. (1).

$$
\mathrm{F}_{0} / \mathrm{F}=1+K_{s v}[\mathrm{C}]=1+K_{q} \tau_{0}[\mathrm{C}]
$$

$\mathrm{F}_{0}$ is $\alpha$-glucosidase fluorescence intensity without adding compounds. $\mathrm{F}$ is $\alpha$ glucosidase fluorescence intensity after adding compounds. $K_{q}$ is the rate constant of the quenching process. $K_{s v}$ is the dynamic quenching constant and $\tau_{0}$ is the average life of $\alpha$-glucosidase in the absence of compounds and the value of $\tau_{0}$ was taken as $10^{-8} \mathrm{~s}$.

Binding constants $(\mathrm{K})$ and binding sites $(\mathrm{n})$. Fluorescence quenching includes static and dynamic types. The binding ratio of small drug molecules to protein molecules in the static quenching is $n: 1$, where the relationship between the fluorescence intensity of $\alpha$-glucosidase and compounds concentration can be derived as Eq. (2). We can also obtain the binding sites (n) from Eq. (2). As static quenching, the combination ratio of $\alpha$-glucosidase and compounds is 1:1. Lineweaver-Burk formula (Eq. (3)) was 
used to calculate binding constant $(\mathrm{K})$.

$$
\begin{aligned}
& \lg \left(F_{0}-F\right) / \mathrm{F}=\lg K+\mathrm{n} \lg [\mathrm{C}] \\
& 1 /\left(F_{0}-F\right)=1 / K F_{0}[\mathrm{C}]+1 / F_{0}
\end{aligned}
$$

Thermodynamics of the $\alpha$-glucosidase- $1 / 2$ complex. Small molecules and protein interact with each other through electrostatic, van der Waals, and hydrophobic forces as well as hydrogen bonds We can regard $\Delta H$ as a constant when temperature changes very slightly. The thermodynamic parameters between compounds and $\alpha$-glucosidase can be calculated by equations (4)-(6).

$$
\begin{aligned}
& \Delta G=-R T \ln K \\
& \Delta S=(\Delta H-\Delta G) / T \\
& \ln \left(K_{2} / K_{1}\right)=\left(1 / T_{1}-1 / T_{2}\right) \Delta H / R
\end{aligned}
$$

$K$ denotes the constant of the binding between $\alpha$-glucosidase and compounds at different temperatures. $\Delta H$ describes the associated change in enthalpy. $\Delta G$ indicates the associated change in free Gibbs energy. $\Delta S$ is the associated change in entropy with reaction. $R=8.314 \mathrm{~J} \mathrm{~mol}^{-1} \mathrm{~K}^{-1}$.

The detailed information of $\mathrm{UV}-\mathrm{Vis}$ spectroscopy measurements. The UV measurements were conducted by the molecular microplate reader (SpectraMax 190, Molecular Devices, Sunnyvale, CA) in 96-well plates at $298 \mathrm{~K}$. The final concentrations of compounds were $0,10,20,30,40,50 \mu \mathrm{M}$, and the final concentration of $\alpha$ glucosidase was $20 \mu \mathrm{M}$. We incubated compounds $\mathbf{1}$ and $\mathbf{2}$ in a normal PBS buffer (pH $7.4,30 \mathrm{~min}$ ) and the recorded spectra range was $200-450 \mathrm{~nm}$. 
Table S1. The inhibitory effect of flavonoids on $\alpha$-glucosidase in previous reports.

\begin{tabular}{lll}
\hline No. & compound & $\mathbf{I C}_{\mathbf{5 0}}(\boldsymbol{\mu M})$ \\
\hline 1 & myricetin & $11.63 \pm 0.36$ \\
2 & luteolin & $82.73 \pm 2.51$ \\
3 & baicalein & $259.56 \pm 3.15$ \\
4 & isorhamnetin & $676.71 \pm 7.42$ \\
5 & quercetin & $115.14 \pm 2.19$ \\
6 & apigenin & $597.56 \pm 5.27$ \\
7 & isorhamnetin-3-O-rutinoside & $539.76 \pm 2.78$ \\
8 & galangin & $415.91 \pm 5.92$ \\
9 & myricitrin & $309.28 \pm 2.40$ \\
10 & vitexin & $356.12 \pm 3.28$ \\
11 & apigenin-7- $O$-glucoside & $22.80 \pm 0.24$ \\
12 & luteoloside & $431.22 \pm 3.03$ \\
13 & vitexin-4'-O-glucoside & $442.50 \pm 3.41$ \\
14 & rutin & $173.58 \pm 1.23$ \\
15 & isoschaftoside & $387.88 \pm 3.68$ \\
16 & kaempferol & $153.39 \pm 1.79$ \\
17 & kaempferol-7- $O$ - $\beta$-glucoside & $204.47 \pm 3.15$ \\
18 & fisetin & $46.39 \pm 0.34$ \\
19 & hesperitin & $1903.27 \pm 5.51$ \\
20 & taxifolin & $2946.97 \pm 4.91$ \\
21 & eriodictyol & $1033.48 \pm 4.74$ \\
22 & genistein & $642.21 \pm 3.66$ \\
23 & formononetin & $747.83 \pm 2.73$ \\
24 & phloretin & $141.10 \pm 2.49$ \\
25 & epigallocatechin gallate & $97.52 \pm 1.35$ \\
26 & cyanidin-3- $O$-glucoside & $63.50 \pm 0.26$ \\
27 & (+)-catechin & $175.1 \pm 9.1$ \\
28 & morin & $4.48 \pm 0.04$ \\
29 & myricetin & $2.25 \pm 0.05$ \\
The data of No.1-26 were obtained from reference $(31)$. & \\
reference (25). The data of No.28-29 were obtained from & \\
\hline & &
\end{tabular}


Table S2. DP4+ probability of ${ }^{13} \mathrm{C}$ NMR data of the isomers (1).

\begin{tabular}{|c|c|c|c|c|c|c|}
\hline Functional & \multicolumn{2}{|c|}{ Solvent? } & \multirow{2}{*}{\multicolumn{2}{|c|}{ Basis Set }} & \multicolumn{2}{|c|}{ Type of Data } \\
\hline mPW1PW91 & \multicolumn{2}{|c|}{ PCM } & & $6-311 G(d, p)$ & \multicolumn{2}{|c|}{ Shielding Tensors } \\
\hline & Isomer 1 & Isomer 2 & Isomer 3 & Isomer 4 & Isomer 5 & Isomer 6 \\
\hline sDP4+ (H data) & - & - & - & - & - & - \\
\hline sDP4+ (C data) & $100.00 \%$ & 메 $0.00 \%$ & - & - & - & - \\
\hline sDP4+ (a11 data) & $100.00 \%$ & ज्ञी $0.00 \%$ & - & - & - & - \\
\hline uDP4+ (H data) & - & - & - & - & - & - \\
\hline uDP4+ (C data) & - $74.63 \%$ & 메 $25.37 \%$ & - & - & - & - \\
\hline uDP4+ (a11 data) & $74.63 \%$ & 메 $25.37 \%$ & - & - & - & - \\
\hline DP4+ (H data) & - & - & - & - & - & - \\
\hline DP4+ (C data) & $100.00 \%$ & 페 $0.00 \%$ & - & - & - & - \\
\hline DP4+ (a11 data) & $\| 100.00 \%$ & 페 $0.00 \%$ & - & - & - & - \\
\hline
\end{tabular}

Table S3. Important thermodynamic parameters (a.u.) and Boltzmann distributions of the optimized $\left(2 R^{*}, 3 R^{*}, 5^{\prime \prime \prime} S^{*}\right)-1$ at B3LYP/6-31G (d) level in methanol.

\begin{tabular}{|c|c|c|}
\hline Conformation & Gibbs free energies (Hartree) & Boltzmann distribution \\
\hline 1 & -2077.151892 & $22.3 \%$ \\
\hline 2 & -2077.150861 & $10.5 \%$ \\
\hline 3 & -2077.150991 & $10.4 \%$ \\
\hline 4 & -2077.151628 & $10.3 \%$ \\
\hline 5 & -2077.152020 & $9.2 \%$ \\
\hline 6 & -2077.152077 & $5.8 \%$ \\
\hline
\end{tabular}

Table S4. Optimized coordinate of $\left(2 R^{*}, 3 R^{*}, 5^{\prime \prime \prime} S^{*}\right)-1$ at B3LYP/6-31G (d) level in methanol.

\begin{tabular}{|c|c|c|c|c|c|c|c|}
\hline \multicolumn{2}{|c|}{ Conformation 1 } & & & & & & \\
\hline Atom & $\mathbf{X}$ & $\mathbf{Y}$ & $\mathbf{Z}$ & $\mathbf{A t o m}$ & $\mathbf{X}$ & $\mathbf{Y}$ & $\mathbf{Z}$ \\
\hline $\mathrm{C}$ & 4.031821 & -0.32055 & 1.974858 & $\mathrm{C}$ & -5.15205 & -2.0862 & -0.94488 \\
\hline $\mathrm{C}$ & 3.398577 & -0.37116 & 3.204635 & $\mathrm{C}$ & -6.52962 & -1.93315 & -0.87976 \\
\hline $\mathrm{C}$ & 1.997135 & -0.37855 & 3.17809 & $\mathrm{C}$ & -7.09574 & -0.98219 & -0.02361 \\
\hline $\mathrm{C}$ & 1.26373 & -0.3359 & 1.978535 & $\mathrm{C}$ & -6.27676 & -0.17589 & 0.776372 \\
\hline $\mathrm{C}$ & 1.966676 & -0.27192 & 0.76307 & $\mathrm{C}$ & -4.89244 & -0.32355 & 0.72079 \\
\hline $\mathrm{C}$ & 3.365619 & -0.27292 & 0.757961 & $\mathrm{O}$ & -6.83317 & 0.749925 & 1.606299 \\
\hline $\mathrm{O}$ & 1.35713 & -0.22811 & -0.45458 & $\mathrm{O}$ & -8.4484 & -0.80052 & 0.068218 \\
\hline $\mathrm{C}$ & -0.24587 & -0.36767 & 1.981058 & $\mathrm{O}$ & -7.44265 & -2.65845 & -1.61037 \\
\hline $\mathrm{C}$ & -0.73883 & -0.84994 & 0.625057 & $\mathrm{H}$ & 3.960833 & -0.39834 & 4.132536 \\
\hline $\mathrm{C}$ & -0.07932 & -0.07757 & -0.54192 & $\mathrm{H}$ & -0.6139 & -1.03312 & 2.76798 \\
\hline $\mathrm{C}$ & -0.49096 & 1.375508 & -0.67744 & $\mathrm{H}$ & -0.66506 & 0.625709 & 2.189266 \\
\hline $\mathrm{O}$ & -2.16796 & -0.68692 & 0.600827 & $\mathrm{H}$ & -0.50778 & -1.90964 & 0.483355 \\
\hline $\mathrm{C}$ & -1.51972 & 1.700855 & -1.5665 & $\mathrm{H}$ & -0.33986 & -0.59963 & -1.46472 \\
\hline $\mathrm{C}$ & -1.95134 & 3.0226 & -1.70499 & $\mathrm{H}$ & -1.98899 & 0.923332 & -2.16243 \\
\hline $\mathrm{C}$ & -1.34525 & 4.029018 & -0.96345 & $\mathrm{H}$ & -2.75274 & 3.268132 & -2.39918 \\
\hline $\mathrm{C}$ & -0.29922 & 3.718836 & -0.07701 & $\mathrm{H}$ & 0.933057 & 2.198201 & 0.750328 \\
\hline $\mathrm{C}$ & 0.119202 & 2.399674 & 0.06263 & $\mathrm{H}$ & -0.12388 & 5.543706 & 0.413067 \\
\hline $\mathrm{O}$ & 0.307465 & 4.703281 & 0.644657 & $\mathrm{H}$ & -2.40989 & 5.490035 & -1.6471 \\
\hline
\end{tabular}




\begin{tabular}{|c|c|c|c|c|c|c|c|}
\hline $\mathrm{O}$ & -1.67936 & 5.36326 & -1.02341 & $\mathrm{H}$ & 3.292795 & 1.435678 & -1.30424 \\
\hline $\mathrm{C}$ & 4.379762 & -0.16678 & -0.34623 & $\mathrm{H}$ & 4.855943 & 1.971106 & -0.66103 \\
\hline $\mathrm{C}$ & 5.703151 & -0.22936 & 0.476042 & $\mathrm{H}$ & 6.053205 & 0.92645 & -2.52754 \\
\hline $\mathrm{O}$ & 5.410393 & -0.29892 & 1.831581 & $\mathrm{H}$ & 4.543222 & 1.29648 & -3.37804 \\
\hline $\mathrm{O}$ & 6.830459 & -0.20393 & 0.071893 & $\mathrm{H}$ & 3.140579 & -2.68635 & -0.57287 \\
\hline $\mathrm{C}$ & 4.34183 & 1.155788 & -1.17652 & $\mathrm{H}$ & 3.905688 & -3.03348 & -2.13128 \\
\hline $\mathrm{C}$ & 4.968931 & 0.77011 & -2.52024 & $\mathrm{H}$ & 4.95324 & -4.41441 & -0.35256 \\
\hline $\mathrm{C}$ & 4.703338 & -0.72836 & -2.63491 & $\mathrm{H}$ & 6.143488 & -3.25759 & -0.9858 \\
\hline $\mathrm{N}$ & 4.34377 & -1.1908 & -1.38505 & $\mathrm{H}$ & 5.372378 & -2.97865 & 0.587827 \\
\hline $\mathrm{C}$ & 4.073928 & -2.60246 & -1.14121 & $\mathrm{H}$ & 1.861287 & -0.47706 & 5.087627 \\
\hline $\mathrm{C}$ & 5.205954 & -3.35062 & -0.42923 & $\mathrm{H}$ & -4.6913 & -2.81691 & -1.60273 \\
\hline $\mathrm{O}$ & 4.79297 & -1.41716 & -3.63712 & $\mathrm{H}$ & -4.26602 & 0.303786 & 1.34195 \\
\hline $\mathrm{O}$ & 1.261036 & -0.42879 & 4.327531 & $\mathrm{H}$ & -7.7994 & 0.69162 & 1.510569 \\
\hline $\mathrm{C}$ & -2.86873 & -1.4865 & -0.24483 & $\mathrm{H}$ & -8.87681 & -1.43414 & -0.53265 \\
\hline $\mathrm{O}$ & -2.3389 & -2.28076 & -1.00105 & $\mathrm{H}$ & -6.97677 & -3.29979 & -2.16794 \\
\hline $\mathrm{C}$ & -4.33413 & -1.27831 & -0.13885 & & & & \\
\hline \multicolumn{8}{|c|}{ Conformation 2} \\
\hline Atom & $\mathbf{X}$ & $\mathbf{Y}$ & $\mathbf{Z}$ & Atom & $\mathbf{X}$ & $\mathbf{Y}$ & $\mathbf{Z}$ \\
\hline $\mathrm{C}$ & 4.025059 & -0.40594 & 1.976093 & $\mathrm{C}$ & -4.8783 & -0.44066 & 0.762715 \\
\hline $\mathrm{C}$ & 3.38478 & -0.52812 & 3.197252 & $\mathrm{C}$ & -6.25831 & -0.30317 & 0.840595 \\
\hline $\mathrm{C}$ & 1.984 & -0.54919 & 3.160587 & $\mathrm{C}$ & -7.09245 & -0.98627 & -0.05047 \\
\hline $\mathrm{C}$ & 1.258384 & -0.45145 & 1.959747 & $\mathrm{C}$ & -6.54357 & -1.81881 & -1.0342 \\
\hline $\mathrm{C}$ & 1.967594 & -0.31682 & 0.753724 & $\mathrm{C}$ & -5.16176 & -1.96346 & -1.12098 \\
\hline $\mathrm{C}$ & 3.366606 & -0.30203 & 0.758295 & $\mathrm{O}$ & -7.35892 & -2.48215 & -1.89921 \\
\hline $\mathrm{O}$ & 1.364356 & -0.21835 & -0.46314 & $\mathrm{O}$ & -8.45478 & -0.87427 & -0.0023 \\
\hline $\mathrm{C}$ & -0.25005 & -0.49724 & 1.950111 & $\mathrm{O}$ & -6.92299 & 0.481521 & 1.756069 \\
\hline $\mathrm{C}$ & -0.73681 & -0.91304 & 0.569634 & $\mathrm{H}$ & 3.941779 & -0.59777 & 4.126115 \\
\hline $\mathrm{C}$ & -0.07392 & -0.08473 & -0.55706 & $\mathrm{H}$ & -0.61687 & -1.20419 & 2.700662 \\
\hline $\mathrm{C}$ & -0.50294 & 1.367218 & -0.63904 & $\mathrm{H}$ & -0.67859 & 0.481373 & 2.205006 \\
\hline $\mathrm{O}$ & -2.16585 & -0.74975 & 0.557111 & $\mathrm{H}$ & -0.50535 & -1.96485 & 0.376984 \\
\hline $\mathrm{C}$ & -1.50773 & 1.718143 & -1.54577 & $\mathrm{H}$ & -0.31812 & -0.57108 & -1.50289 \\
\hline $\mathrm{C}$ & -1.95026 & 3.04003 & -1.64332 & $\mathrm{H}$ & -1.94845 & 0.960869 & -2.18779 \\
\hline $\mathrm{C}$ & -1.37849 & 4.021187 & -0.84293 & $\mathrm{H}$ & -2.73222 & 3.305569 & -2.35223 \\
\hline $\mathrm{C}$ & -0.35737 & 3.685458 & 0.063146 & $\mathrm{H}$ & 0.870043 & 2.145025 & 0.862039 \\
\hline $\mathrm{C}$ & 0.071892 & 2.365962 & 0.161776 & $\mathrm{H}$ & -0.20033 & 5.494949 & 0.612869 \\
\hline $\mathrm{O}$ & 0.215298 & 4.646158 & 0.842791 & $\mathrm{H}$ & -2.38771 & 5.510689 & -1.54925 \\
\hline $\mathrm{O}$ & -1.72752 & 5.353377 & -0.85769 & $\mathrm{H}$ & 3.290273 & 1.510759 & -1.21647 \\
\hline $\mathrm{C}$ & 4.387572 & -0.12594 & -0.33084 & $\mathrm{H}$ & 4.837852 & 2.032063 & -0.52507 \\
\hline $\mathrm{C}$ & 5.705728 & -0.21651 & 0.497389 & $\mathrm{H}$ & 6.07357 & 1.100052 & -2.42589 \\
\hline $\mathrm{O}$ & 5.403834 & -0.36092 & 1.84527 & $\mathrm{H}$ & 4.571635 & 1.499647 & -3.27759 \\
\hline $\mathrm{O}$ & 6.835622 & -0.15633 & 0.104575 & $\mathrm{H}$ & 3.177759 & -2.6426 & -0.70546 \\
\hline $\mathrm{C}$ & 4.341535 & 1.238627 & -1.08985 & $\mathrm{H}$ & 3.96234 & -2.89773 & -2.27175 \\
\hline $\mathrm{C}$ & 4.991188 & 0.932455 & -2.44312 & $\mathrm{H}$ & 5.005856 & -4.36048 & -0.5566 \\
\hline $\mathrm{C}$ & 4.744241 & -0.56044 & -2.64165 & $\mathrm{H}$ & 6.190398 & -3.16151 & -1.11743 \\
\hline $\mathrm{N}$ & 4.372183 & -1.09271 & -1.42334 & $\mathrm{H}$ & 5.402334 & -2.97183 & 0.461257 \\
\hline $\mathrm{C}$ & 4.115848 & -2.51845 & -1.25839 & $\mathrm{H}$ & 1.837919 & -0.7554 & 5.060429 \\
\hline $\mathrm{C}$ & 5.248785 & -3.29184 & -0.5754 & $\mathrm{H}$ & -4.22815 & 0.089311 & 1.450521 \\
\hline
\end{tabular}




\begin{tabular}{|c|c|c|c|c|c|c|c|}
\hline $\mathrm{O}$ & 4.855776 & -1.19367 & -3.67747 & $\mathrm{H}$ & -4.72998 & -2.60766 & -1.87768 \\
\hline $\mathrm{O}$ & 1.241017 & -0.66655 & 4.301332 & $\mathrm{H}$ & -8.27808 & -2.25856 & -1.67283 \\
\hline $\mathrm{C}$ & -2.86753 & -1.46927 & -0.36157 & $\mathrm{H}$ & -8.68077 & -0.27847 & 0.732462 \\
\hline $\mathrm{O}$ & -2.33429 & -2.17986 & -1.19246 & $\mathrm{H}$ & -6.28229 & 0.904244 & 2.347499 \\
\hline $\mathrm{C}$ & -4.3325 & -1.2769 & -0.22524 & & & & \\
\hline \multicolumn{8}{|c|}{ Conformation 3} \\
\hline Atom & $\mathbf{X}$ & $\mathbf{Y}$ & $\mathbf{Z}$ & Atom & $\mathbf{X}$ & $\mathbf{Y}$ & $\mathbf{Z}$ \\
\hline $\mathrm{C}$ & -4.15389 & -0.84002 & -1.83177 & $\mathrm{C}$ & 4.787316 & -0.72461 & -0.72344 \\
\hline $\mathrm{C}$ & -3.53725 & -1.30593 & -2.98013 & $\mathrm{C}$ & 6.174404 & -0.657 & -0.83325 \\
\hline $\mathrm{C}$ & -2.1357 & -1.33103 & -2.96196 & $\mathrm{C}$ & 6.979078 & -1.12021 & 0.214894 \\
\hline $\mathrm{C}$ & -1.38668 & -0.9066 & -1.84972 & $\mathrm{C}$ & 6.395906 & -1.64918 & 1.371507 \\
\hline $\mathrm{C}$ & -2.07283 & -0.43099 & -0.71866 & $\mathrm{C}$ & 5.015525 & -1.721 & 1.490956 \\
\hline $\mathrm{C}$ & -3.47166 & -0.40448 & -0.70389 & $\mathrm{O}$ & 7.296229 & -2.068 & 2.324151 \\
\hline $\mathrm{O}$ & -1.44698 & 0.002507 & 0.410172 & $\mathrm{O}$ & 8.335178 & -1.03235 & 0.059857 \\
\hline $\mathrm{C}$ & 0.121833 & -0.96914 & -1.84828 & $\mathrm{O}$ & 6.747759 & -0.14218 & -1.95656 \\
\hline $\mathrm{C}$ & 0.620691 & -0.99627 & -0.41108 & $\mathrm{H}$ & -4.11174 & -1.62929 & -3.84229 \\
\hline $\mathrm{C}$ & -0.00565 & 0.13437 & 0.439415 & $\mathrm{H}$ & 0.469177 & -1.86086 & -2.37885 \\
\hline $\mathrm{C}$ & 0.441591 & 1.53974 & 0.083948 & $\mathrm{H}$ & 0.557679 & -0.10513 & -2.36732 \\
\hline $\mathrm{O}$ & 2.052845 & -0.87742 & -0.45048 & $\mathrm{H}$ & 0.363092 & -1.9442 & 0.070478 \\
\hline $\mathrm{C}$ & -0.17032 & 2.297765 & -0.91987 & $\mathrm{H}$ & 0.25129 & -0.06058 & 1.482232 \\
\hline $\mathrm{C}$ & 0.292373 & 3.584155 & -1.21398 & $\mathrm{H}$ & -1.01565 & 1.904091 & -1.47319 \\
\hline $\mathrm{C}$ & 1.367178 & 4.116273 & -0.51252 & $\mathrm{H}$ & -0.18985 & 4.173588 & -1.99169 \\
\hline $\mathrm{C}$ & 1.987845 & 3.366665 & 0.501711 & $\mathrm{H}$ & 2.000435 & 1.539031 & 1.595345 \\
\hline $\mathrm{C}$ & 1.516965 & 2.091753 & 0.79514 & $\mathrm{H}$ & 3.201495 & 4.780357 & 0.860666 \\
\hline $\mathrm{O}$ & 3.032954 & 3.886251 & 1.204298 & $\mathrm{H}$ & 1.410173 & 5.821465 & -1.42141 \\
\hline $\mathrm{O}$ & 1.900078 & 5.36893 & -0.71865 & $\mathrm{H}$ & -3.34966 & 1.876855 & 0.699388 \\
\hline $\mathrm{C}$ & -4.47027 & 0.071077 & 0.313718 & $\mathrm{H}$ & -4.91427 & 2.204068 & -0.07074 \\
\hline $\mathrm{C}$ & -5.80473 & -0.23135 & -0.43428 & $\mathrm{H}$ & -6.10138 & 1.839953 & 2.041523 \\
\hline $\mathrm{O}$ & -5.53016 & -0.74804 & -1.69383 & $\mathrm{H}$ & -4.57362 & 2.430361 & 2.717296 \\
\hline $\mathrm{O}$ & -6.9263 & -0.05276 & -0.05367 & $\mathrm{H}$ & -3.26334 & -2.26391 & 1.333381 \\
\hline $\mathrm{C}$ & -4.40417 & 1.589073 & 0.675348 & $\mathrm{H}$ & -4.02061 & -2.07928 & 2.922625 \\
\hline $\mathrm{C}$ & -5.0203 & 1.666367 & 2.076245 & $\mathrm{H}$ & -5.10685 & -3.93833 & 1.686382 \\
\hline $\mathrm{C}$ & -4.77874 & 0.278787 & 2.663377 & $\mathrm{H}$ & -6.27205 & -2.617 & 1.915121 \\
\hline $\mathrm{N}$ & -4.43615 & -0.5655 & 1.626345 & $\mathrm{H}$ & -5.50887 & -2.87497 & 0.334076 \\
\hline $\mathrm{C}$ & -4.19059 & -1.98557 & 1.847167 & $\mathrm{H}$ & -2.02633 & -2.07167 & -4.72674 \\
\hline $\mathrm{C}$ & -5.34066 & -2.9021 & 1.41645 & $\mathrm{H}$ & 4.172469 & -0.36085 & -1.53668 \\
\hline $\mathrm{O}$ & -4.87209 & -0.05134 & 3.83324 & $\mathrm{H}$ & 4.542023 & -2.12785 & 2.379334 \\
\hline $\mathrm{O}$ & -1.41548 & -1.77215 & -4.03569 & $\mathrm{H}$ & 6.818029 & -2.40339 & 3.097473 \\
\hline $\mathrm{C}$ & 2.743268 & -1.35702 & 0.618194 & $\mathrm{H}$ & 8.75144 & -1.38255 & 0.866178 \\
\hline $\mathrm{O}$ & 2.198923 & -1.81333 & 1.607609 & $\mathrm{H}$ & 7.712678 & -0.17669 & -1.83883 \\
\hline $\mathrm{C}$ & 4.211717 & -1.25614 & 0.437803 & & & & \\
\hline \multicolumn{8}{|c|}{ Conformation 4} \\
\hline Atom & $\mathbf{X}$ & $\mathbf{Y}$ & $\mathbf{Z}$ & Atom & $\mathbf{X}$ & $\mathbf{Y}$ & $\mathbf{Z}$ \\
\hline $\mathrm{C}$ & 4.070309 & -0.16105 & 2.049908 & $\mathrm{C}$ & -4.85259 & -0.20256 & 0.768034 \\
\hline $\mathrm{C}$ & 3.430792 & -0.08167 & 3.274837 & $\mathrm{C}$ & -6.23559 & -0.0366 & 0.800659 \\
\hline $\mathrm{C}$ & 2.029776 & -0.0817 & 3.241422 & $\mathrm{C}$ & -7.05737 & -0.89046 & 0.054705 \\
\hline $\mathrm{C}$ & 1.303279 & -0.154 & 2.039092 & $\mathrm{C}$ & -6.49519 & -1.90766 & -0.72443 \\
\hline
\end{tabular}




\begin{tabular}{|c|c|c|c|c|c|c|c|}
\hline $\mathrm{C}$ & 2.012507 & -0.22005 & 0.827466 & $\mathrm{C}$ & -5.11899 & -2.07993 & -0.76579 \\
\hline $\mathrm{C}$ & 3.412133 & -0.23793 & 0.829502 & $\mathrm{O}$ & -7.41028 & -2.6747 & -1.40834 \\
\hline $\mathrm{O}$ & 1.4058 & -0.28947 & -0.39099 & $\mathrm{O}$ & -8.40853 & -0.68798 & 0.121017 \\
\hline $\mathrm{C}$ & -0.20687 & -0.1656 & 2.036861 & $\mathrm{O}$ & -6.78815 & 0.95324 & 1.555824 \\
\hline $\mathrm{C}$ & -0.70207 & -0.77374 & 0.733768 & $\mathrm{H}$ & 3.988705 & -0.01482 & 4.203335 \\
\hline $\mathrm{C}$ & -0.02835 & -0.12767 & -0.49983 & $\mathrm{H}$ & -0.58707 & -0.74335 & 2.884861 \\
\hline $\mathrm{C}$ & -0.41947 & 1.310343 & -0.7793 & $\mathrm{H}$ & -0.61248 & 0.849537 & 2.141693 \\
\hline $\mathrm{O}$ & -2.12921 & -0.59745 & 0.689323 & $\mathrm{H}$ & -0.48382 & -1.84513 & 0.697784 \\
\hline $\mathrm{C}$ & -1.44202 & 1.558799 & -1.69993 & $\mathrm{H}$ & -0.29201 & -0.73373 & -1.36855 \\
\hline $\mathrm{C}$ & -1.85708 & 2.865541 & -1.96938 & $\mathrm{H}$ & -1.91921 & 0.731702 & -2.2177 \\
\hline $\mathrm{C}$ & -1.24064 & 3.932678 & -1.32801 & $\mathrm{H}$ & -2.65375 & 3.051292 & -2.68717 \\
\hline $\mathrm{C}$ & -0.20062 & 3.698875 & -0.41154 & $\mathrm{H}$ & 1.010323 & 2.252424 & 0.566645 \\
\hline $\mathrm{C}$ & 0.201188 & 2.3949 & -0.14107 & $\mathrm{H}$ & -0.00365 & 5.560948 & -0.1032 \\
\hline $\mathrm{O}$ & 0.416116 & 4.742207 & 0.212167 & $\mathrm{H}$ & -2.2863 & 5.331819 & -2.15529 \\
\hline $\mathrm{O}$ & -1.55824 & 5.258348 & -1.52025 & $\mathrm{H}$ & 3.330741 & 1.206938 & -1.41973 \\
\hline $\mathrm{C}$ & 4.43564 & -0.24984 & -0.27239 & $\mathrm{H}$ & 4.88149 & 1.839153 & -0.84037 \\
\hline $\mathrm{C}$ & 5.752195 & -0.21665 & 0.56336 & $\mathrm{H}$ & 6.099569 & 0.631063 & -2.5845 \\
\hline $\mathrm{O}$ & 5.449853 & -0.15426 & 1.915568 & $\mathrm{H}$ & 4.572674 & 0.82467 & -3.45949 \\
\hline $\mathrm{O}$ & 6.882168 & -0.23913 & 0.166267 & $\mathrm{H}$ & 5.2072 & -3.3224 & -1.17744 \\
\hline $\mathrm{C}$ & 4.38292 & 0.960252 & -1.25711 & $\mathrm{H}$ & 4.475269 & -2.80292 & 0.348899 \\
\hline $\mathrm{C}$ & 5.023318 & 0.430301 & -2.54513 & $\mathrm{H}$ & 3.027672 & -4.49706 & -0.7883 \\
\hline $\mathrm{C}$ & 4.83061 & -1.08148 & -2.46276 & $\mathrm{H}$ & 2.184574 & -2.93009 & -0.74202 \\
\hline $\mathrm{N}$ & 4.43902 & -1.39328 & -1.17946 & $\mathrm{H}$ & 2.964001 & -3.47716 & -2.24071 \\
\hline $\mathrm{C}$ & 4.358264 & -2.7864 & -0.7397 & $\mathrm{H}$ & 1.883393 & 0.017855 & 5.150082 \\
\hline $\mathrm{C}$ & 3.048537 & -3.46265 & -1.15069 & $\mathrm{H}$ & -4.22452 & 0.462051 & 1.347451 \\
\hline $\mathrm{O}$ & 5.00687 & -1.9004 & -3.34982 & $\mathrm{H}$ & -4.66147 & -2.86195 & -1.36424 \\
\hline $\mathrm{O}$ & 1.28749 & -0.00893 & 4.385504 & $\mathrm{H}$ & -6.9468 & -3.35861 & -1.91499 \\
\hline $\mathrm{C}$ & -2.83465 & -1.45489 & -0.09327 & $\mathrm{H}$ & -8.83933 & -1.35925 & -0.43563 \\
\hline $\mathrm{O}$ & -2.30949 & -2.31281 & -0.78018 & $\mathrm{H}$ & -7.75419 & 0.897909 & 1.456459 \\
\hline $\mathrm{C}$ & -4.2982 & -1.22372 & -0.01436 & & & & \\
\hline \multicolumn{8}{|c|}{ Conformation 5} \\
\hline Atom & $\mathbf{X}$ & $\mathbf{Y}$ & $\mathbf{Z}$ & Atom & $\mathbf{X}$ & $\mathbf{Y}$ & $\mathbf{Z}$ \\
\hline $\mathrm{C}$ & 4.259349 & -0.85157 & 1.661702 & $\mathrm{C}$ & -4.49266 & -0.23579 & -0.01813 \\
\hline $\mathrm{C}$ & 3.693382 & -1.46732 & 2.764362 & $\mathrm{C}$ & -5.77807 & 0.200661 & -0.3023 \\
\hline $\mathrm{C}$ & 2.294919 & -1.56447 & 2.768721 & $\mathrm{C}$ & -6.75655 & -0.72034 & -0.69041 \\
\hline $\mathrm{C}$ & 1.499529 & -1.06392 & 1.721499 & $\mathrm{C}$ & -6.43982 & -2.08201 & -0.81016 \\
\hline $\mathrm{C}$ & 2.13551 & -0.43644 & 0.638041 & $\mathrm{C}$ & -5.13868 & -2.51833 & -0.56704 \\
\hline $\mathrm{C}$ & 3.530142 & -0.33432 & 0.599264 & $\mathrm{O}$ & -7.39912 & -2.97031 & -1.19493 \\
\hline $\mathrm{O}$ & 1.462523 & 0.086358 & -0.4285 & $\mathrm{O}$ & -8.03644 & -0.33501 & -0.98132 \\
\hline $\mathrm{C}$ & -0.00302 & -1.20986 & 1.737464 & $\mathrm{O}$ & -6.17594 & 1.512945 & -0.23676 \\
\hline $\mathrm{C}$ & -0.5365 & -1.13201 & 0.311908 & $\mathrm{H}$ & 4.303362 & -1.85078 & 3.575991 \\
\hline $\mathrm{C}$ & 0.022794 & 0.101779 & -0.4448 & $\mathrm{H}$ & -0.29168 & -2.16541 & 2.185022 \\
\hline $\mathrm{C}$ & -0.54206 & 1.429322 & 0.038434 & $\mathrm{H}$ & -0.47424 & -0.42139 & 2.339582 \\
\hline $\mathrm{O}$ & -1.95742 & -0.98243 & 0.316829 & $\mathrm{H}$ & -0.27311 & -2.03899 & -0.23973 \\
\hline $\mathrm{C}$ & 0.081499 & 2.214898 & 1.01039 & $\mathrm{H}$ & -0.2313 & -0.01504 & -1.50275 \\
\hline $\mathrm{C}$ & -0.53222 & 3.381857 & 1.480324 & $\mathrm{H}$ & 1.046848 & 1.929132 & 1.413445 \\
\hline $\mathrm{C}$ & -1.78478 & 3.752282 & 1.004442 & $\mathrm{H}$ & -0.03875 & 3.992918 & 2.233381 \\
\hline
\end{tabular}




\begin{tabular}{|c|c|c|c|c|c|c|c|}
\hline $\mathrm{C}$ & -2.41648 & 2.969876 & 0.024853 & $\mathrm{H}$ & -2.27603 & 1.271143 & -1.25241 \\
\hline $\mathrm{C}$ & -1.77905 & 1.845669 & -0.47851 & $\mathrm{H}$ & -3.90026 & 4.168224 & -0.01523 \\
\hline $\mathrm{O}$ & -3.67863 & 3.30849 & -0.41618 & $\mathrm{H}$ & -1.98305 & 5.357372 & 2.066407 \\
\hline $\mathrm{O}$ & -2.49489 & 4.856565 & 1.413471 & $\mathrm{H}$ & 3.273799 & 2.069929 & -0.56956 \\
\hline $\mathrm{C}$ & 4.481211 & 0.288158 & -0.38411 & $\mathrm{H}$ & 4.835729 & 2.389706 & 0.20972 \\
\hline $\mathrm{C}$ & 5.84599 & -0.02344 & 0.30367 & $\mathrm{H}$ & 6.000693 & 2.287057 & -1.94398 \\
\hline $\mathrm{O}$ & 5.625858 & -0.67829 & 1.507977 & $\mathrm{H}$ & 4.440028 & 2.883869 & -2.53461 \\
\hline $\mathrm{O}$ & 6.948107 & 0.249089 & -0.07788 & $\mathrm{H}$ & 3.369705 & -1.99334 & -1.60281 \\
\hline $\mathrm{C}$ & 4.340486 & 1.830037 & -0.58818 & $\mathrm{H}$ & 4.058448 & -1.60766 & -3.1877 \\
\hline $\mathrm{C}$ & 4.926463 & 2.076293 & -1.98207 & $\mathrm{H}$ & 5.27902 & -3.52205 & -2.18371 \\
\hline $\mathrm{C}$ & 4.727771 & 0.74661 & -2.70493 & $\mathrm{H}$ & 6.368436 & -2.12489 & -2.31243 \\
\hline $\mathrm{N}$ & 4.443225 & -0.21316 & -1.75411 & $\mathrm{H}$ & 5.677681 & -2.58124 & -0.74271 \\
\hline $\mathrm{C}$ & 4.262113 & -1.61475 & -2.114 & $\mathrm{H}$ & 2.262376 & -2.50712 & 4.43791 \\
\hline $\mathrm{C}$ & 5.471366 & -2.50761 & -1.81623 & $\mathrm{H}$ & -3.74714 & 0.470654 & 0.316443 \\
\hline $\mathrm{O}$ & 4.806893 & 0.542327 & -3.90404 & $\mathrm{H}$ & -4.88392 & -3.5637 & -0.69735 \\
\hline $\mathrm{O}$ & 1.62275 & -2.1521 & 3.80124 & $\mathrm{H}$ & -8.22181 & -2.47219 & -1.33995 \\
\hline $\mathrm{C}$ & -2.75964 & -2.04149 & -0.0149 & $\mathrm{H}$ & -8.06935 & 0.635375 & -0.91185 \\
\hline $\mathrm{O}$ & -2.34084 & -3.16794 & -0.18025 & $\mathrm{H}$ & -5.38399 & 2.086863 & -0.2546 \\
\hline $\mathrm{C}$ & -4.16413 & -1.5911 & -0.17679 & & & & \\
\hline \multicolumn{8}{|c|}{ Conformation 6} \\
\hline Atom & $\mathbf{X}$ & $\mathbf{Y}$ & $\mathbf{Z}$ & Atom & $\mathbf{X}$ & $\mathbf{Y}$ & $\mathbf{Z}$ \\
\hline $\mathrm{C}$ & -4.17248 & -0.77657 & -1.84568 & $\mathrm{C}$ & 4.999299 & -1.82293 & 1.412982 \\
\hline $\mathrm{C}$ & -3.56341 & -1.21404 & -3.00916 & $\mathrm{C}$ & 6.379896 & -1.73166 & 1.306801 \\
\hline $\mathrm{C}$ & -2.16191 & -1.24554 & -2.99915 & $\mathrm{C}$ & 6.964173 & -1.10305 & 0.201518 \\
\hline $\mathrm{C}$ & -1.40562 & -0.85579 & -1.87906 & $\mathrm{C}$ & 6.16028 & -0.56135 & -0.8094 \\
\hline $\mathrm{C}$ & -2.08443 & -0.40881 & -0.73235 & $\mathrm{C}$ & 4.772859 & -0.65091 & -0.71418 \\
\hline $\mathrm{C}$ & -3.48259 & -0.37484 & -0.70991 & $\mathrm{O}$ & 6.733349 & 0.050507 & -1.88201 \\
\hline $\mathrm{O}$ & -1.45094 & -0.01125 & 0.406636 & $\mathrm{O}$ & 8.319687 & -0.9913 & 0.061217 \\
\hline $\mathrm{C}$ & 0.102915 & -0.92372 & -1.88711 & $\mathrm{O}$ & 7.27898 & -2.21913 & 2.227052 \\
\hline $\mathrm{C}$ & 0.604309 & -1.00072 & -0.4531 & $\mathrm{H}$ & -4.14352 & -1.51098 & -3.87703 \\
\hline $\mathrm{C}$ & -0.01031 & 0.110052 & 0.43186 & $\mathrm{H}$ & 0.444824 & -1.79818 & -2.44888 \\
\hline $\mathrm{C}$ & 0.446537 & 1.520139 & 0.111792 & $\mathrm{H}$ & 0.538945 & -0.04328 & -2.37758 \\
\hline $\mathrm{O}$ & 2.03886 & -0.88947 & -0.48846 & $\mathrm{H}$ & 0.341838 & -1.96136 & -0.00066 \\
\hline $\mathrm{C}$ & -0.17867 & 2.327021 & -0.84442 & $\mathrm{H}$ & 0.250737 & -0.11752 & 1.467603 \\
\hline $\mathrm{C}$ & 0.292213 & 3.614849 & -1.11228 & $\mathrm{H}$ & -1.04611 & 1.96355 & -1.38466 \\
\hline $\mathrm{C}$ & 1.399369 & 4.114242 & -0.4337 & $\mathrm{H}$ & -0.19229 & 4.246944 & -1.84976 \\
\hline $\mathrm{C}$ & 2.0303 & 3.309094 & 0.530724 & $\mathrm{H}$ & 2.043377 & 1.43431 & 1.570627 \\
\hline $\mathrm{C}$ & 1.55373 & 2.034507 & 0.805366 & $\mathrm{H}$ & 3.52189 & 3.256588 & 1.759518 \\
\hline $\mathrm{O}$ & 3.10714 & 3.893764 & 1.158828 & $\mathrm{H}$ & 2.633575 & 5.520723 & -0.12596 \\
\hline $\mathrm{O}$ & 1.859443 & 5.369007 & -0.69515 & $\mathrm{H}$ & -3.34277 & 1.875875 & 0.736502 \\
\hline $\mathrm{C}$ & -4.4735 & 0.081947 & 0.323595 & $\mathrm{H}$ & -4.91218 & 2.224458 & -0.01304 \\
\hline $\mathrm{C}$ & -5.81321 & -0.19896 & -0.42314 & $\mathrm{H}$ & -6.08277 & 1.820892 & 2.10147 \\
\hline $\mathrm{O}$ & -5.54738 & -0.68274 & -1.69774 & $\mathrm{H}$ & -4.54733 & 2.392877 & 2.775221 \\
\hline $\mathrm{O}$ & -6.9319 & -0.02906 & -0.03056 & $\mathrm{H}$ & -3.27219 & -2.27953 & 1.283474 \\
\hline $\mathrm{C}$ & -4.39842 & 1.592097 & 0.715688 & $\mathrm{H}$ & -4.01314 & -2.1235 & 2.883621 \\
\hline $\mathrm{C}$ & -5.002 & 1.643057 & 2.123175 & $\mathrm{H}$ & -5.12001 & -3.95216 & 1.62054 \\
\hline $\mathrm{C}$ & -4.76006 & 0.243136 & 2.679825 & $\mathrm{H}$ & -6.27656 & -2.63057 & 1.888427 \\
\hline
\end{tabular}




\begin{tabular}{|l|c|c|c|c|c|c|c|}
\hline $\mathrm{N}$ & -4.43363 & -0.58192 & 1.62234 & $\mathrm{H}$ & -5.53115 & -2.85941 & 0.294629 \\
\hline $\mathrm{C}$ & -4.19303 & -2.00723 & 1.811958 & $\mathrm{H}$ & -2.06412 & -1.9284 & -4.78777 \\
\hline $\mathrm{C}$ & -5.35175 & -2.90954 & 1.374342 & $\mathrm{H}$ & 4.524906 & -2.30514 & 2.262401 \\
\hline $\mathrm{O}$ & -4.84093 & -0.11014 & 3.844 & $\mathrm{H}$ & 4.159167 & -0.22999 & -1.50049 \\
\hline $\mathrm{O}$ & -1.44887 & -1.66044 & -4.08773 & $\mathrm{H}$ & 7.698619 & 0.003499 & -1.77123 \\
\hline $\mathrm{C}$ & 2.726854 & -1.4075 & 0.561354 & $\mathrm{H}$ & 8.737322 & -1.42601 & 0.824585 \\
\hline $\mathrm{O}$ & 2.184567 & -1.91025 & 1.529468 & $\mathrm{H}$ & 6.802211 & -2.66706 & 2.942133 \\
\hline $\mathrm{C}$ & 4.196288 & -1.28185 & 0.395898 & & & & \\
\hline
\end{tabular}

Table S5. Optimized coordinate of $\left(2 R^{*}, 3 R^{*}, 5^{\prime \prime \prime} R^{*}\right)-1$ at B3LYP/6-31G (d) level in methanol.

\begin{tabular}{|c|c|c|c|c|c|c|c|}
\hline \multicolumn{8}{|c|}{ Conformation 1} \\
\hline Atom & $\mathbf{X}$ & $\mathbf{Y}$ & $\mathbf{Z}$ & Atom & $\mathbf{X}$ & $\mathbf{Y}$ & $\mathbf{Z}$ \\
\hline $\mathrm{C}$ & -3.70816 & -2.37303 & -0.74562 & $\mathrm{C}$ & 5.105366 & -0.48897 & -0.669 \\
\hline $\mathrm{C}$ & -3.05176 & -3.13192 & -1.69666 & $\mathrm{C}$ & 6.433508 & -0.2039 & -0.97838 \\
\hline $\mathrm{C}$ & -1.65868 & -2.98167 & -1.74713 & $\mathrm{C}$ & 7.352665 & 0.033368 & 0.050955 \\
\hline $\mathrm{C}$ & -0.95739 & -2.14028 & -0.86532 & $\mathrm{C}$ & 6.942259 & -0.01642 & 1.387691 \\
\hline $\mathrm{C}$ & -1.68166 & -1.41305 & 0.093764 & $\mathrm{C}$ & 5.622217 & -0.30135 & 1.706206 \\
\hline $\mathrm{C}$ & -3.07887 & -1.50751 & 0.138711 & $\mathrm{O}$ & 7.940447 & 0.234422 & 2.300768 \\
\hline $\mathrm{O}$ & -1.07027 & -0.64232 & 1.037444 & $\mathrm{O}$ & 8.643988 & 0.310127 & -0.30375 \\
\hline $\mathrm{C}$ & 0.546003 & -2.01677 & -0.96197 & $\mathrm{O}$ & 6.837126 & -0.15718 & -2.27811 \\
\hline $\mathrm{C}$ & 1.108681 & -1.33936 & 0.282759 & $\mathrm{H}$ & -3.58968 & -3.79208 & -2.36943 \\
\hline $\mathrm{C}$ & 0.26838 & -0.13178 & 0.733595 & $\mathrm{H}$ & 1.005677 & -3.00345 & -1.08322 \\
\hline $\mathrm{C}$ & 0.14125 & 1.087361 & -0.16629 & $\mathrm{H}$ & 0.815022 & -1.43941 & -1.85421 \\
\hline $\mathrm{O}$ & 2.475606 & -0.97929 & 0.002656 & $\mathrm{H}$ & 1.108834 & -2.03578 & 1.126007 \\
\hline $\mathrm{C}$ & 0.88772 & 1.34968 & -1.31744 & $\mathrm{H}$ & 0.631657 & 0.1721 & 1.718288 \\
\hline $\mathrm{C}$ & 0.644282 & 2.506585 & -2.07033 & $\mathrm{H}$ & 1.67441 & 0.675466 & -1.6336 \\
\hline $\mathrm{C}$ & -0.35575 & 3.398543 & -1.6974 & $\mathrm{H}$ & 1.218805 & 2.716052 & -2.96737 \\
\hline $\mathrm{C}$ & -1.10365 & 3.146624 & -0.52989 & $\mathrm{H}$ & -1.38711 & 1.867579 & 1.144431 \\
\hline $\mathrm{C}$ & -0.82719 & 2.02364 & 0.232277 & $\mathrm{H}$ & -2.68292 & 3.684378 & 0.455439 \\
\hline $\mathrm{O}$ & -2.0879 & 4.052367 & -0.23811 & $\mathrm{H}$ & -1.36231 & 4.963981 & -2.01224 \\
\hline $\mathrm{O}$ & -0.62133 & 4.504681 & -2.44695 & $\mathrm{H}$ & -3.42064 & -1.64672 & 2.826923 \\
\hline $\mathrm{C}$ & -4.13988 & -0.78662 & 0.936097 & $\mathrm{H}$ & -5.07514 & -1.00251 & 2.875644 \\
\hline $\mathrm{C}$ & -5.42199 & -1.50326 & 0.414555 & $\mathrm{H}$ & -4.05399 & 0.957847 & 3.795863 \\
\hline $\mathrm{O}$ & -5.08963 & -2.38684 & -0.58907 & $\mathrm{H}$ & -2.48094 & 0.566765 & 3.090444 \\
\hline $\mathrm{O}$ & -6.55169 & -1.34925 & 0.783932 & $\mathrm{H}$ & -3.785 & 2.015037 & -0.85463 \\
\hline $\mathrm{C}$ & -4.06504 & -0.83229 & 2.491725 & $\mathrm{H}$ & -4.31956 & 0.431175 & -1.40413 \\
\hline $\mathrm{C}$ & -3.56057 & 0.556528 & 2.906772 & $\mathrm{H}$ & -6.09707 & 2.158974 & -1.79114 \\
\hline $\mathrm{C}$ & -3.84204 & 1.442383 & 1.704107 & $\mathrm{H}$ & -6.67836 & 0.984947 & -0.58931 \\
\hline $\mathrm{N}$ & -4.2218 & 0.658132 & 0.660207 & $\mathrm{H}$ & -6.08588 & 2.574988 & -0.06679 \\
\hline $\mathrm{C}$ & -4.50732 & 1.216975 & -0.66625 & $\mathrm{H}$ & -1.47506 & -4.21585 & -3.20354 \\
\hline $\mathrm{C}$ & -5.93362 & 1.762053 & -0.78292 & $\mathrm{H}$ & 4.404622 & -0.67406 & -1.47301 \\
\hline $\mathrm{O}$ & -3.70628 & 2.668202 & 1.653925 & $\mathrm{H}$ & 5.283301 & -0.34664 & 2.736705 \\
\hline $\mathrm{O}$ & -0.89899 & -3.66071 & -2.65542 & $\mathrm{H}$ & 7.57584 & 0.200464 & 3.19803 \\
\hline $\mathrm{C}$ & 3.306177 & -0.83695 & 1.07141 & $\mathrm{H}$ & 9.153281 & 0.459132 & 0.511398 \\
\hline $\mathrm{O}$ & 2.927232 & -0.94881 & 2.222976 & $\mathrm{H}$ & 7.7851 & 0.060575 & -2.28729 \\
\hline $\mathrm{C}$ & 4.702251 & -0.53583 & 0.671809 & & & & \\
\hline Conforr & on 2 & & & & & & \\
\hline
\end{tabular}




\begin{tabular}{|c|c|c|c|c|c|c|c|}
\hline Atom & $\mathbf{X}$ & $\mathbf{Y}$ & $\mathbf{Z}$ & Atom & $\mathbf{X}$ & $\mathbf{Y}$ & $\mathbf{Z}$ \\
\hline $\mathrm{C}$ & -3.82375 & -2.34337 & -0.64405 & $\mathrm{C}$ & 5.014527 & -0.57046 & -0.66556 \\
\hline $\mathrm{C}$ & -3.18206 & -3.15114 & -1.56468 & $\mathrm{C}$ & 6.344083 & -0.30842 & -0.98889 \\
\hline $\mathrm{C}$ & -1.78732 & -3.01857 & -1.63106 & $\mathrm{C}$ & 7.273825 & -0.06722 & 0.029989 \\
\hline $\mathrm{C}$ & -1.07035 & -2.15005 & -0.78919 & $\mathrm{C}$ & 6.87248 & -0.09 & 1.370212 \\
\hline $\mathrm{C}$ & -1.78028 & -1.37755 & 0.144784 & $\mathrm{C}$ & 5.551086 & -0.3519 & 1.702604 \\
\hline $\mathrm{C}$ & -3.17822 & -1.44639 & 0.194562 & $\mathrm{O}$ & 7.880505 & 0.162125 & 2.272077 \\
\hline $\mathrm{O}$ & -1.15621 & -0.58407 & 1.060278 & $\mathrm{O}$ & 8.56608 & 0.186688 & -0.3381 \\
\hline $\mathrm{C}$ & 0.432988 & -2.04285 & -0.90039 & $\mathrm{O}$ & 6.738677 & -0.28761 & -2.29205 \\
\hline $\mathrm{C}$ & 1.013477 & -1.34917 & 0.327414 & $\mathrm{H}$ & -3.73135 & -3.83293 & -2.20603 \\
\hline $\mathrm{C}$ & 0.200828 & -0.11795 & 0.764915 & $\mathrm{H}$ & 0.882555 & -3.0358 & -1.0065 \\
\hline $\mathrm{C}$ & 0.119394 & 1.108022 & -0.1298 & $\mathrm{H}$ & 0.700653 & -1.48534 & -1.80568 \\
\hline $\mathrm{O}$ & 2.384036 & -1.01687 & 0.032016 & $\mathrm{H}$ & 1.007766 & -2.03123 & 1.18243 \\
\hline $\mathrm{C}$ & 0.832371 & 1.324736 & -1.31134 & $\mathrm{H}$ & 0.564418 & 0.177004 & 1.752141 \\
\hline $\mathrm{C}$ & 0.629992 & 2.494407 & -2.05651 & $\mathrm{H}$ & 1.563491 & 0.60441 & -1.65783 \\
\hline $\mathrm{C}$ & -0.29956 & 3.444697 & -1.64594 & $\mathrm{H}$ & 1.179469 & 2.668693 & -2.97644 \\
\hline $\mathrm{C}$ & -1.0175 & 3.237167 & -0.45044 & $\mathrm{H}$ & -1.30632 & 1.98025 & 1.240115 \\
\hline $\mathrm{C}$ & -0.77336 & 2.102173 & 0.305954 & $\mathrm{H}$ & -2.58165 & 3.825038 & 0.518175 \\
\hline $\mathrm{O}$ & -1.93253 & 4.198869 & -0.12065 & $\mathrm{H}$ & -1.23364 & 5.058197 & -1.92767 \\
\hline $\mathrm{O}$ & -0.52977 & 4.564166 & -2.38613 & $\mathrm{H}$ & -3.55544 & -1.52291 & 2.868673 \\
\hline $\mathrm{C}$ & -4.22649 & -0.66795 & 0.955986 & $\mathrm{H}$ & -5.15993 & -0.75852 & 2.905768 \\
\hline $\mathrm{C}$ & -5.52229 & -1.37075 & 0.457289 & $\mathrm{H}$ & -3.96462 & 1.137358 & 3.771605 \\
\hline $\mathrm{O}$ & -5.20553 & -2.31992 & -0.49004 & $\mathrm{H}$ & -2.4533 & 0.635357 & 2.999544 \\
\hline $\mathrm{O}$ & -6.65059 & -1.15644 & 0.799479 & $\mathrm{H}$ & -5.77747 & 0.954111 & -0.79176 \\
\hline $\mathrm{C}$ & -4.14264 & -0.67513 & 2.511163 & $\mathrm{H}$ & -4.84928 & 2.409613 & -0.43055 \\
\hline $\mathrm{C}$ & -3.53922 & 0.687464 & 2.870799 & $\mathrm{H}$ & -4.29911 & 1.64737 & -2.69494 \\
\hline $\mathrm{C}$ & -3.83964 & 1.563606 & 1.665363 & $\mathrm{H}$ & -2.86002 & 1.438886 & -1.67881 \\
\hline $\mathrm{N}$ & -4.27996 & 0.77253 & 0.645272 & $\mathrm{H}$ & -3.84519 & 0.035604 & -2.12907 \\
\hline $\mathrm{C}$ & -4.76421 & 1.336797 & -0.6191 & $\mathrm{H}$ & -1.6289 & -4.31498 & -3.03528 \\
\hline $\mathrm{C}$ & -3.88246 & 1.091276 & -1.84734 & $\mathrm{H}$ & 4.305341 & -0.75804 & -1.46151 \\
\hline $\mathrm{O}$ & -3.69158 & 2.786902 & 1.607439 & $\mathrm{H}$ & 5.219087 & -0.37585 & 2.73607 \\
\hline $\mathrm{O}$ & -1.04224 & -3.7432 & -2.51632 & $\mathrm{H}$ & 7.5219 & 0.147951 & 3.172283 \\
\hline $\mathrm{C}$ & 3.223411 & -0.86572 & 1.092584 & $\mathrm{H}$ & 9.082826 & 0.342993 & 0.470972 \\
\hline $\mathrm{O}$ & 2.850497 & -0.95115 & 2.248412 & $\mathrm{H}$ & 7.689241 & -0.08217 & -2.31159 \\
\hline $\mathrm{C}$ & 4.620595 & -0.59054 & 0.678618 & & & & \\
\hline \multicolumn{8}{|c|}{ Conformation 3} \\
\hline Atom & $\mathbf{X}$ & $\mathbf{Y}$ & $\mathbf{Z}$ & Atom & $\mathbf{X}$ & $\mathbf{Y}$ & $\mathbf{Z}$ \\
\hline $\mathrm{C}$ & -3.70558 & -2.35655 & -0.79255 & $\mathrm{C}$ & 5.636938 & -0.41876 & 1.714923 \\
\hline $\mathrm{C}$ & -3.05095 & -3.09251 & -1.76278 & $\mathrm{C}$ & 6.961632 & -0.11342 & 1.41639 \\
\hline $\mathrm{C}$ & -1.65855 & -2.93796 & -1.81481 & $\mathrm{C}$ & 7.3515 & 0.045327 & 0.08043 \\
\hline $\mathrm{C}$ & -0.95574 & -2.11496 & -0.91711 & $\mathrm{C}$ & 6.416572 & -0.10391 & -0.94912 \\
\hline $\mathrm{C}$ & -1.67804 & -1.41119 & 0.061183 & $\mathrm{C}$ & 5.092418 & -0.41018 & -0.66179 \\
\hline $\mathrm{C}$ & -3.07485 & -1.50968 & 0.108639 & $\mathrm{O}$ & 6.929566 & 0.073768 & -2.2141 \\
\hline $\mathrm{O}$ & -1.06576 & -0.66046 & 1.020085 & $\mathrm{O}$ & 8.662147 & 0.347186 & -0.16604 \\
\hline $\mathrm{C}$ & 0.547413 & -1.98721 & -1.01568 & $\mathrm{O}$ & 7.874344 & 0.032595 & 2.415453 \\
\hline $\mathrm{C}$ & 1.111204 & -1.34162 & 0.245045 & $\mathrm{H}$ & -3.59007 & -3.73849 & -2.44824 \\
\hline $\mathrm{C}$ & 0.272483 & -0.14471 & 0.725794 & $\mathrm{H}$ & 1.007185 & -2.97043 & -1.16244 \\
\hline
\end{tabular}




\begin{tabular}{|c|c|c|c|c|c|c|c|}
\hline $\mathrm{C}$ & 0.142988 & 1.094724 & -0.14603 & $\mathrm{H}$ & 0.812255 & -1.38742 & -1.89436 \\
\hline $\mathrm{O}$ & 2.478782 & -0.97411 & -0.01947 & $\mathrm{H}$ & 1.108137 & -2.05914 & 1.070095 \\
\hline $\mathrm{C}$ & 0.887295 & 1.382832 & -1.29221 & $\mathrm{H}$ & 0.638919 & 0.136439 & 1.716201 \\
\hline $\mathrm{C}$ & 0.641511 & 2.554948 & -2.02037 & $\mathrm{H}$ & 1.673738 & 0.715113 & -1.62245 \\
\hline $\mathrm{C}$ & -0.35901 & 3.437606 & -1.62741 & $\mathrm{H}$ & 1.213523 & 2.785057 & -2.9141 \\
\hline $\mathrm{C}$ & -1.10523 & 3.159622 & -0.46471 & $\mathrm{H}$ & -1.38536 & 1.844899 & 1.181979 \\
\hline $\mathrm{C}$ & -0.8262 & 2.020977 & 0.273089 & $\mathrm{H}$ & -2.68267 & 3.674804 & 0.535229 \\
\hline $\mathrm{O}$ & -2.09033 & 4.056965 & -0.15323 & $\mathrm{H}$ & -1.36619 & 5.009296 & -1.90682 \\
\hline $\mathrm{O}$ & -0.62669 & 4.558764 & -2.35321 & $\mathrm{H}$ & -3.40992 & -1.70643 & 2.795697 \\
\hline $\mathrm{C}$ & -4.13466 & -0.80783 & 0.924757 & $\mathrm{H}$ & -5.06402 & -1.06268 & 2.861909 \\
\hline $\mathrm{C}$ & -5.41706 & -1.51572 & 0.392261 & $\mathrm{H}$ & -4.03863 & 0.877384 & 3.819598 \\
\hline $\mathrm{O}$ & -5.08616 & -2.37655 & -0.63198 & $\mathrm{H}$ & -2.46856 & 0.501426 & 3.099776 \\
\hline $\mathrm{O}$ & -6.54579 & -1.37252 & 0.76847 & $\mathrm{H}$ & -3.79227 & 2.032685 & -0.80775 \\
\hline $\mathrm{C}$ & -4.05491 & -0.88523 & 2.478691 & $\mathrm{H}$ & -4.32285 & 0.458796 & -1.38934 \\
\hline $\mathrm{C}$ & -3.54892 & 0.494847 & 2.920274 & $\mathrm{H}$ & -6.10727 & 2.18848 & -1.73443 \\
\hline $\mathrm{C}$ & -3.83571 & 1.405114 & 1.737385 & $\mathrm{H}$ & -6.6812 & 0.986916 & -0.55657 \\
\hline $\mathrm{N}$ & -4.21947 & 0.642308 & 0.678918 & $\mathrm{H}$ & -6.09282 & 2.567376 & -0.00159 \\
\hline $\mathrm{C}$ & -4.51113 & 1.228099 & -0.63436 & $\mathrm{H}$ & -1.47804 & -4.14414 & -3.29461 \\
\hline $\mathrm{C}$ & -5.93965 & 1.770554 & -0.73542 & $\mathrm{H}$ & 5.327656 & -0.541 & 2.746019 \\
\hline $\mathrm{O}$ & -3.70057 & 2.631763 & 1.711341 & $\mathrm{H}$ & 4.368102 & -0.53247 & -1.4599 \\
\hline $\mathrm{O}$ & -0.90098 & -3.59446 & -2.74201 & $\mathrm{H}$ & 6.218227 & -0.00058 & -2.86774 \\
\hline $\mathrm{C}$ & 3.309254 & -0.88966 & 1.061285 & $\mathrm{H}$ & 8.773055 & 0.440018 & -1.12784 \\
\hline $\mathrm{O}$ & 2.924526 & -1.06676 & 2.200842 & $\mathrm{H}$ & 8.730757 & 0.247019 & 2.007175 \\
\hline $\mathrm{C}$ & 4.704952 & -0.56498 & 0.679671 & & & & \\
\hline \multicolumn{8}{|c|}{ Conformation 4} \\
\hline Atom & $\mathbf{X}$ & $\mathbf{Y}$ & $\mathbf{Z}$ & Atom & $\mathbf{X}$ & $\mathbf{Y}$ & $\mathbf{Z}$ \\
\hline $\mathrm{C}$ & -3.71378 & -2.39911 & -0.67394 & $\mathrm{C}$ & 5.104592 & -0.47584 & -0.6728 \\
\hline $\mathrm{C}$ & -3.0718 & -3.16288 & -1.62727 & $\mathrm{C}$ & 6.433481 & -0.1955 & -0.98266 \\
\hline $\mathrm{C}$ & -1.68052 & -2.99881 & -1.71305 & $\mathrm{C}$ & 7.361296 & 0.003233 & 0.04724 \\
\hline $\mathrm{C}$ & -0.96228 & -2.14844 & -0.84784 & $\mathrm{C}$ & 6.958513 & -0.07989 & 1.384897 \\
\hline $\mathrm{C}$ & -1.67394 & -1.42368 & 0.124934 & $\mathrm{C}$ & 5.637583 & -0.3605 & 1.70369 \\
\hline $\mathrm{C}$ & -3.065 & -1.52445 & 0.19045 & $\mathrm{O}$ & 7.964729 & 0.134966 & 2.297359 \\
\hline $\mathrm{O}$ & -1.05877 & -0.64701 & 1.063224 & $\mathrm{O}$ & 8.652436 & 0.276464 & -0.30804 \\
\hline $\mathrm{C}$ & 0.538016 & -1.98187 & -0.97023 & $\mathrm{O}$ & 6.828932 & -0.11666 & -2.28299 \\
\hline $\mathrm{C}$ & 1.109123 & -1.3222 & 0.282465 & $\mathrm{H}$ & -3.59423 & -3.83255 & -2.29868 \\
\hline $\mathrm{C}$ & 0.267178 & -0.12408 & 0.757988 & $\mathrm{H}$ & 1.043214 & -2.95042 & -1.09842 \\
\hline $\mathrm{C}$ & 0.129329 & 1.115632 & -0.11364 & $\mathrm{H}$ & 0.787674 & -1.3676 & -1.84572 \\
\hline $\mathrm{O}$ & 2.474672 & -0.96786 & -0.00066 & $\mathrm{H}$ & 1.107763 & -2.03396 & 1.112562 \\
\hline $\mathrm{C}$ & 0.933788 & 1.439542 & -1.20859 & $\mathrm{H}$ & 0.642858 & 0.161091 & 1.744098 \\
\hline $\mathrm{C}$ & 0.714828 & 2.625756 & -1.9221 & $\mathrm{H}$ & 1.748451 & 0.791232 & -1.50838 \\
\hline $\mathrm{C}$ & -0.31237 & 3.490273 & -1.56049 & $\mathrm{H}$ & 1.333713 & 2.882846 & -2.77621 \\
\hline $\mathrm{C}$ & -1.12284 & 3.175852 & -0.45013 & $\mathrm{H}$ & -1.49756 & 1.798236 & 1.13255 \\
\hline $\mathrm{C}$ & -0.88058 & 2.016614 & 0.269938 & $\mathrm{H}$ & -2.76817 & 3.683401 & 0.464303 \\
\hline $\mathrm{O}$ & -2.12399 & 4.066854 & -0.17741 & $\mathrm{H}$ & -1.31552 & 5.061551 & -1.85189 \\
\hline $\mathrm{O}$ & -0.54742 & 4.629656 & -2.26771 & $\mathrm{H}$ & -2.94177 & -0.9394 & 2.771164 \\
\hline $\mathrm{C}$ & -4.09868 & -0.74269 & 0.95098 & $\mathrm{H}$ & -4.63556 & -1.41555 & 2.975507 \\
\hline $\mathrm{C}$ & -5.39655 & -1.47385 & 0.49855 & $\mathrm{H}$ & -5.33227 & 0.878532 & 3.225804 \\
\hline
\end{tabular}




\begin{tabular}{|c|c|c|c|c|c|c|c|}
\hline $\mathrm{O}$ & -5.09247 & -2.39171 & -0.49172 & $\mathrm{H}$ & -3.64739 & 1.164948 & 3.677533 \\
\hline $\mathrm{O}$ & -6.51268 & -1.31097 & 0.901583 & $\mathrm{H}$ & -3.77262 & 2.160478 & -0.81979 \\
\hline $\mathrm{C}$ & -3.97003 & -0.6892 & 2.504577 & $\mathrm{H}$ & -3.43909 & 0.533094 & -1.39993 \\
\hline $\mathrm{C}$ & -4.29383 & 0.760698 & 2.894865 & $\mathrm{H}$ & -5.46416 & 1.549403 & -2.49586 \\
\hline $\mathrm{C}$ & -4.12154 & 1.549054 & 1.608321 & $\mathrm{H}$ & -5.93279 & 0.100631 & -1.59997 \\
\hline $\mathrm{N}$ & -4.14819 & 0.680885 & 0.558493 & $\mathrm{H}$ & -6.25403 & 1.705137 & -0.91459 \\
\hline $\mathrm{C}$ & -4.15272 & 1.138969 & -0.83431 & $\mathrm{H}$ & -0.10832 & -3.52015 & -2.69033 \\
\hline $\mathrm{C}$ & -5.53664 & 1.114463 & -1.49271 & $\mathrm{H}$ & 4.397628 & -0.63013 & -1.47781 \\
\hline $\mathrm{O}$ & -3.98235 & 2.771782 & 1.519604 & $\mathrm{H}$ & 5.304831 & -0.43128 & 2.734724 \\
\hline $\mathrm{O}$ & -1.05579 & -3.72402 & -2.68326 & $\mathrm{H}$ & 7.606728 & 0.08062 & 3.196326 \\
\hline $\mathrm{C}$ & 3.313904 & -0.85609 & 1.068729 & $\mathrm{H}$ & 9.169315 & 0.398033 & 0.506988 \\
\hline $\mathrm{O}$ & 2.937022 & -0.99321 & 2.217497 & $\mathrm{H}$ & 7.778844 & 0.092719 & -2.29336 \\
\hline $\mathrm{C}$ & 4.708989 & -0.55659 & 0.668821 & & & & \\
\hline \multicolumn{8}{|c|}{ Conformation 5} \\
\hline Atom & $\mathbf{X}$ & $\mathbf{Y}$ & $\mathbf{Z}$ & Atom & $\mathbf{X}$ & $\mathbf{Y}$ & $\mathbf{Z}$ \\
\hline $\mathrm{C}$ & -3.5393 & -2.42052 & -0.89419 & $\mathrm{C}$ & 4.950608 & 0.611998 & 0.151806 \\
\hline $\mathrm{C}$ & -2.79457 & -3.07607 & -1.8567 & $\mathrm{C}$ & 6.218993 & 1.181991 & 0.061446 \\
\hline $\mathrm{C}$ & -1.41418 & -2.83115 & -1.83725 & $\mathrm{C}$ & 7.356118 & 0.380745 & 0.223382 \\
\hline $\mathrm{C}$ & -0.80807 & -1.99814 & -0.87956 & $\mathrm{C}$ & 7.223615 & -0.98883 & 0.476628 \\
\hline $\mathrm{C}$ & -1.61932 & -1.37854 & 0.08475 & $\mathrm{C}$ & 5.965447 & -1.56644 & 0.5686 \\
\hline $\mathrm{C}$ & -3.00742 & -1.5681 & 0.06398 & $\mathrm{O}$ & 8.415815 & -1.66209 & 0.615504 \\
\hline $\mathrm{O}$ & -1.10057 & -0.62288 & 1.096818 & $\mathrm{O}$ & 8.577937 & 0.987283 & 0.125029 \\
\hline $\mathrm{C}$ & 0.687443 & -1.77544 & -0.89956 & $\mathrm{O}$ & 6.353051 & 2.514472 & -0.18281 \\
\hline $\mathrm{C}$ & 1.146292 & -1.1271 & 0.405825 & $\mathrm{H}$ & -3.25855 & -3.72931 & -2.58869 \\
\hline $\mathrm{C}$ & 0.207897 & -0.0085 & 0.875862 & $\mathrm{H}$ & 1.216336 & -2.72348 & -1.036 \\
\hline $\mathrm{C}$ & 0.031739 & 1.24646 & 0.034851 & $\mathrm{H}$ & 0.957292 & -1.13747 & -1.74928 \\
\hline $\mathrm{O}$ & 2.471676 & -0.57706 & 0.292652 & $\mathrm{H}$ & 1.16118 & -1.88662 & 1.192101 \\
\hline $\mathrm{C}$ & 0.805747 & 1.619716 & -1.06641 & $\mathrm{H}$ & 0.508642 & 0.275826 & 1.889511 \\
\hline $\mathrm{C}$ & 0.516332 & 2.798096 & -1.7679 & $\mathrm{H}$ & 1.645195 & 1.013851 & -1.38534 \\
\hline $\mathrm{C}$ & -0.55572 & 3.601425 & -1.39362 & $\mathrm{H}$ & 1.111869 & 3.093666 & -2.62611 \\
\hline $\mathrm{C}$ & -1.33373 & 3.236527 & -0.27653 & $\mathrm{H}$ & -1.60053 & 1.850279 & 1.313219 \\
\hline $\mathrm{C}$ & -1.01407 & 2.093231 & 0.437361 & $\mathrm{H}$ & -2.98715 & 3.613845 & 0.660342 \\
\hline $\mathrm{O}$ & -2.38801 & 4.058639 & 0.017077 & $\mathrm{H}$ & -1.64812 & 5.116008 & -1.66765 \\
\hline $\mathrm{O}$ & -0.86301 & 4.728007 & -2.09434 & $\mathrm{H}$ & -3.45971 & -1.87389 & 2.726532 \\
\hline $\mathrm{C}$ & -4.148 & -0.9631 & 0.847929 & $\mathrm{H}$ & -5.15251 & -1.33598 & 2.72673 \\
\hline $\mathrm{C}$ & -5.35523 & -1.7331 & 0.232575 & $\mathrm{H}$ & -4.29829 & 0.631047 & 3.792616 \\
\hline $\mathrm{O}$ & -4.92221 & -2.53441 & -0.80077 & $\mathrm{H}$ & -2.67651 & 0.380707 & 3.134584 \\
\hline $\mathrm{O}$ & -6.50691 & -1.67498 & 0.559327 & $\mathrm{H}$ & -3.90881 & 1.94123 & -0.78963 \\
\hline $\mathrm{C}$ & -4.13907 & -1.0844 & 2.400713 & $\mathrm{H}$ & -4.32187 & 0.356956 & -1.43459 \\
\hline $\mathrm{C}$ & -3.7453 & 0.310061 & 2.905996 & $\mathrm{H}$ & -6.18801 & 1.993964 & -1.80997 \\
\hline $\mathrm{C}$ & -4.03829 & 1.237661 & 1.73779 & $\mathrm{H}$ & -6.73931 & 0.722904 & -0.69589 \\
\hline $\mathrm{N}$ & -4.31699 & 0.485854 & 0.639863 & $\mathrm{H}$ & -6.27035 & 2.316069 & -0.0675 \\
\hline $\mathrm{C}$ & -4.58643 & 1.092409 & -0.66889 & $\mathrm{H}$ & -1.08637 & -3.97008 & -3.34472 \\
\hline $\mathrm{C}$ & -6.03892 & 1.55403 & -0.81753 & $\mathrm{H}$ & 4.078566 & 1.242235 & 0.02931 \\
\hline $\mathrm{O}$ & -3.98658 & 2.470859 & 1.756015 & $\mathrm{H}$ & 5.839912 & -2.62694 & 0.765021 \\
\hline $\mathrm{O}$ & -0.57413 & -3.40263 & -2.74799 & $\mathrm{H}$ & 8.240455 & -2.59884 & 0.791206 \\
\hline $\mathrm{C}$ & 3.509174 & -1.43193 & 0.512707 & $\mathrm{H}$ & 9.261222 & 0.308459 & 0.260325 \\
\hline
\end{tabular}




\begin{tabular}{|c|c|c|c|c|c|c|c|}
\hline $\mathrm{O}$ & 3.352392 & -2.61113 & 0.76769 & $\mathrm{H}$ & 7.303755 & 2.718757 & -0.20589 \\
\hline $\mathrm{C}$ & 4.827335 & -0.7608 & 0.403778 & & & & \\
\hline \multicolumn{8}{|c|}{ Conformation 6} \\
\hline Atom & $\mathbf{X}$ & $\mathbf{Y}$ & $\mathbf{Z}$ & Atom & $\mathbf{X}$ & $\mathbf{Y}$ & $\mathbf{Z}$ \\
\hline $\mathrm{C}$ & -3.57382 & -2.40739 & -0.87144 & $\mathrm{C}$ & 4.908155 & 0.574103 & 0.162967 \\
\hline $\mathrm{C}$ & -2.83938 & -3.09259 & -1.82119 & $\mathrm{C}$ & 6.165244 & 1.158559 & 0.075538 \\
\hline $\mathrm{C}$ & -1.45401 & -2.87728 & -1.79941 & $\mathrm{C}$ & 7.321084 & 0.384997 & 0.225513 \\
\hline $\mathrm{C}$ & -0.83379 & -2.04328 & -0.85124 & $\mathrm{C}$ & 7.223708 & -0.99125 & 0.467587 \\
\hline $\mathrm{C}$ & -1.63574 & -1.39352 & 0.100457 & $\mathrm{C}$ & 5.968886 & -1.58687 & 0.558732 \\
\hline $\mathrm{C}$ & -3.02725 & -1.55357 & 0.077072 & $\mathrm{O}$ & 8.351629 & -1.73963 & 0.613022 \\
\hline $\mathrm{O}$ & -1.10564 & -0.63367 & 1.103923 & $\mathrm{O}$ & 8.573712 & 0.927816 & 0.145301 \\
\hline $\mathrm{C}$ & 0.666042 & -1.85041 & -0.87077 & $\mathrm{O}$ & 6.399975 & 2.494733 & -0.15495 \\
\hline $\mathrm{C}$ & 1.137448 & -1.18308 & 0.421188 & $\mathrm{H}$ & -3.31437 & -3.74605 & -2.54584 \\
\hline $\mathrm{C}$ & 0.213232 & -0.04763 & 0.878401 & $\mathrm{H}$ & 1.17742 & -2.81068 & -0.98656 \\
\hline $\mathrm{C}$ & 0.061512 & 1.203479 & 0.025685 & $\mathrm{H}$ & 0.94977 & -1.23676 & -1.73389 \\
\hline $\mathrm{O}$ & 2.462301 & -0.63837 & 0.292392 & $\mathrm{H}$ & 1.152976 & -1.92977 & 1.219816 \\
\hline $\mathrm{C}$ & 0.823591 & 1.54035 & -1.0957 & $\mathrm{H}$ & 0.518025 & 0.243082 & 1.88902 \\
\hline $\mathrm{C}$ & 0.553283 & 2.717265 & -1.80794 & $\mathrm{H}$ & 1.635484 & 0.903176 & -1.42472 \\
\hline $\mathrm{C}$ & -0.48946 & 3.554517 & -1.42503 & $\mathrm{H}$ & 1.135078 & 2.981734 & -2.68579 \\
\hline $\mathrm{C}$ & -1.25716 & 3.224695 & -0.28982 & $\mathrm{H}$ & -1.5319 & 1.867735 & 1.323521 \\
\hline $\mathrm{C}$ & -0.95361 & 2.083622 & 0.434967 & $\mathrm{H}$ & -2.89902 & 3.643276 & 0.647786 \\
\hline $\mathrm{O}$ & -2.28533 & 4.076159 & 0.009168 & $\mathrm{H}$ & -1.55439 & 5.086657 & -1.70723 \\
\hline $\mathrm{O}$ & -0.77921 & 4.679801 & -2.13498 & $\mathrm{H}$ & -3.49518 & -1.82747 & 2.738686 \\
\hline $\mathrm{C}$ & -4.15651 & -0.91577 & 0.850848 & $\mathrm{H}$ & -5.17548 & -1.25209 & 2.729387 \\
\hline $\mathrm{C}$ & -5.37864 & -1.6639 & 0.237574 & $\mathrm{H}$ & -4.28321 & 0.704347 & 3.781852 \\
\hline $\mathrm{O}$ & -4.95925 & -2.48907 & -0.78244 & $\mathrm{H}$ & -2.66386 & 0.411425 & 3.136017 \\
\hline $\mathrm{O}$ & -6.5303 & -1.57336 & 0.556762 & $\mathrm{H}$ & -3.84556 & 1.97212 & -0.80485 \\
\hline $\mathrm{C}$ & -4.15573 & -1.02555 & 2.40479 & $\mathrm{H}$ & -4.28705 & 0.392394 & -1.44218 \\
\hline $\mathrm{C}$ & -3.73254 & 0.363536 & 2.901178 & $\mathrm{H}$ & -6.11727 & 2.065269 & -1.83861 \\
\hline $\mathrm{C}$ & -3.99754 & 1.288317 & 1.724307 & $\mathrm{H}$ & -6.70073 & 0.812299 & -0.72058 \\
\hline $\mathrm{N}$ & -4.29096 & 0.535328 & 0.631448 & $\mathrm{H}$ & -6.20333 & 2.399153 & -0.09846 \\
\hline $\mathrm{C}$ & -4.54106 & 1.13806 & -0.6828 & $\mathrm{H}$ & -1.1421 & -4.04889 & -3.28565 \\
\hline $\mathrm{C}$ & -5.98293 & 1.628264 & -0.84278 & $\mathrm{H}$ & 4.010812 & 1.173575 & 0.050813 \\
\hline $\mathrm{O}$ & -3.91553 & 2.520366 & 1.733806 & $\mathrm{H}$ & 5.885398 & -2.65047 & 0.747911 \\
\hline $\mathrm{O}$ & -0.6226 & -3.47809 & -2.69842 & $\mathrm{H}$ & 9.116219 & -1.14594 & 0.517898 \\
\hline $\mathrm{C}$ & 3.505086 & -1.49189 & 0.516316 & $\mathrm{H}$ & 8.476764 & 1.881578 & -0.01915 \\
\hline $\mathrm{O}$ & 3.349296 & -2.66852 & 0.774718 & $\mathrm{H}$ & 5.556466 & 2.964677 & -0.23862 \\
\hline $\mathrm{C}$ & 4.81578 & -0.80696 & 0.405478 & & & & \\
\hline
\end{tabular}

